University of Wollongong

Research Online

Australian Institute for Innovative Materials -

Papers

Australian Institute for Innovative Materials

$1-1-2018$

Electrochemical and Electrostatic Cleavage of Alkoxyamines

Long Zhang

University of Wollongong, Iz898@uowmail.edu.au

Eduardo Laborda

University of Murcia

Nadim A. Darwish

Curtin University, nadimdarwish@gmail.com

Benjamin B. Noble

Australian National University

Jason Tyrell

Australian National University

See next page for additional authors

Follow this and additional works at: https://ro.uow.edu.au/aiimpapers

Part of the Engineering Commons, and the Physical Sciences and Mathematics Commons

Research Online is the open access institutional repository for the University of Wollongong. For further information contact the UOW Library: research-pubs@uow.edu.au 


\title{
Electrochemical and Electrostatic Cleavage of Alkoxyamines
}

\author{
Abstract \\ Alkoxyamines are heat-labile molecules, widely used as an in situ source of nitroxides in polymer and \\ materials sciences. Here we show that the one-electron oxidation of an alkoxyamine leads to a cation \\ radical intermediate that even at room temperature rapidly fragments, releasing a nitroxide and \\ carbocation. Digital simulations of experimental voltammetry and current-time transients suggest that the \\ unimolecular decomposition which yields the "unmasked" nitroxide (TEMPO) is exceedingly rapid and \\ irreversible. High-level quantum computations indicate that the collapse of the alkoxyamine cation radical \\ is likely to yield a neutral nitroxide radical and a secondary phenylethyl cation. However, this \\ fragmentation is predicted to be slow and energetically very unfavorable. To attain qualitative agreement \\ between the experimental kinetics and computational modeling for this fragmentation step, the explicit \\ electrostatic environment within the double layer must be accounted for. Single-molecule break-junction \\ experiments in a scanning tunneling microscope using solvent of low dielectric (STM-BJ technique) \\ corroborate the role played by electrostatic forces on the lysis of the alkoxyamine C-ON bond. This work \\ highlights the electrostatic aspects played by charged species in a chemical step that follows an \\ electrochemical reaction, defines the magnitude of this catalytic effect by looking at an independent \\ electrical technique in non-electrolyte systems (STM-BJ), and suggests a redox on/off switch to guide the \\ cleavage of alkoxyamines at an electrified interface.

\section{Disciplines} \\ Engineering | Physical Sciences and Mathematics \\ Publication Details \\ Zhang, L., Laborda, E., Darwish, N., Noble, B. B., Tyrell, J. H., Pluczyk, S., Le Brun, A. P., Wallace, G. G., \\ Gonzalez, J., Coote, M. L. \& Ciampi, S. (2018). Electrochemical and Electrostatic Cleavage of \\ Alkoxyamines. Journal of the American Chemical Society, 140 (2), 766-774.

\section{Authors} \\ Long Zhang, Eduardo Laborda, Nadim A. Darwish, Benjamin B. Noble, Jason Tyrell, Sandra Pluczyk, Anton \\ P. Le Brun, Gordon G. Wallace, Joaquin Gonzalez, M Coote, and Simone Ciampi
}




\title{
Electrochemical and Electrostatic Cleavage of Alkoxyamines
}

\author{
Long Zhang, ${ }^{\mathrm{a}, \mathrm{b}}$ Eduardo Laborda, ${ }^{\mathrm{c}}$ Nadim Darwish, ${ }^{*, \mathrm{a}}$ Benjamin B. Noble, ${ }^{\mathrm{d}}$ Jason H. Tyrell, ${ }^{\mathrm{d}}$ San- \\ dra Pluczyk, ${ }^{\mathrm{e}}$ Anton P. Le Brun, ${ }^{\mathrm{f}}$ Gordon G. Wallace, ${ }^{\mathrm{b}}$ Joaquin Gonzalez, ${ }^{*, \mathrm{c}}$ Michelle L. Coote, ${ }^{*, \mathrm{~d}}$ \\ and Simone Ciampi ${ }^{*}$,a
}

\author{
${ }^{a}$ Department of Chemistry, Curtin University, Bentley, Western Australia 6102, Australia \\ ${ }^{b}$ ARC Centre of Excellence for Electromaterials Science, Intelligent Polymer Research Institute, University of Wol- \\ longong, Wollongong, New South Wales 2500, Australia \\ ${ }^{\mathrm{c}}$ Department de Quimica Fisica, Universidad De Murcia, Murcia 30003, Spain \\ ${ }^{\mathrm{d}}$ ARC Centre of Excellence for Electromaterials Science, Research School of Chemistry, Australian National Univer- \\ sity, Canberra, Australian Capital Territory 2601, Australia \\ ${ }^{\mathrm{e}}$ Faculty of Chemistry, Silesian University of Technology, Gliwice 44-100, Poland \\ ${ }^{\mathrm{f}}$ Australian Centre for Neutron Scattering, Australian Nuclear Science and Technology Organization (ANSTO), \\ Lucas Heights, NSW 2234, Australia
}

Supporting Information Placeholder

\begin{abstract}
Alkoxyamines are heat-labile molecules, widely used as in-situ source of nitroxides in polymer and materials sciences. Here we show that the one-electron oxidation of an alkoxyamine leads to a cation radical intermediate that even at room temperature rapidly fragments releasing a nitroxide and carbocation. Digital simulations of experimental voltammetry and current-time transients suggest the unimolecular decomposition which yields the "unmasked" nitroxide (TEMPO) is exceedingly rapid and irreversible. High-level quantum computations indicate the collapse of the alkoxyamine cation radical is likely to yield a neutral nitroxide radical and a secondary phenylethyl cation. However, this fragmentation is predicted to be slow and energetically very unfavorable. To attain qualitative agreement between the experimental kinetics and computational modelling for this fragmentation step the explicit electrostatic environment within the double layer must be accounted for. Single-molecule break-junction experiments in a scanning tunneling microscope using solvent of low dielectric (STM-BJ technique) corroborate the role played by electrostatics forces on the lysis of the alkoxyamine $\mathrm{C}-\mathrm{ON}$ bond. This work highlights the electrostatic aspects played by charged species in a chemical step that follows an electrochemical reaction, defines the magnitude of this catalytic effect by looking at an independent electrical technique in non-electrolyte systems (STM-BJ), and suggests a redox on/off switch to guide the cleavage of alkoxyamines at an electrified interface.
\end{abstract}

\section{INTRODUCTION}

Electrostatic catalysis refers to the use of static electric fields to catalyse ordinary chemical reactions. ${ }^{1}$ Conversion of reactants into products is synonymous of electronic reorganization, yet little is known on the effect of static charges on chemical catalysis. Chemists appreciate that redox currents at electrodes respond predictably to changes in voltage - for instance a bias of about one Volt pointing in the right direction can lead to changes in redox currents by a factor of up to $10^{8}$. These currents are the manifestation of the rate at which electrons are either lost or gained; hence the field-effect is easily explained and accounted for. On the other hand, the effect of static electricity on non-redox reactivity, long suggested by theoretical chemists, ${ }^{2}$ is not intuitive and it is just starting now to emerge as an important branch of chemical catalysis. ${ }^{1,3}$ The chemical implications are expected to be broad since electrolytes and electrostatic interactions are ubiquitous in chemical, material and biological sciences, ${ }^{2 c}$,
${ }^{4}$ to the point of dominating the entire reaction space in natural or technological environments, such as cell membranes, ${ }^{5}$ fluidic channels ${ }^{6}$ or the confined electrolyte space of a porous electrode. ${ }^{7}$

The theories and models describing the role of electrostatic forces on the formation or rupture of chemical bonds were pioneered by Pocker, ${ }^{8}$ Bertrán, ${ }^{2 \mathrm{C}}$ Warshel ${ }^{9}$ and Shaik ${ }^{10}$ starting from the 1970s. This form of catalysis arises because formally covalent species can be stabilized via minor charge-separated resonance contributors. A non-polar covalent bond A-B might be written as $[\mathrm{A}-\mathrm{B} \leftrightarrow$ $\left.A^{+}-B^{-} \leftrightarrow A^{-}-B^{+}\right]$, but in the absence of an electrostatic force A-B is dominant and the extent of resonance stabilization is small. Only in the presence of an appropriatelyoriented electric field one of the charge-separated contributors can be stabilized. The electrostatic "awakening" of the ionic character of bonds, and the effect on reaction barriers that follows can be dramatic., ${ }^{13}{ }^{\mathrm{b}}{ }^{11}$ The obvious and perceived challenge is how to align molecules and field so as to take advantage of these effects..$^{\text {b, } 12}$ 
The present experiments were initially designed as an attempt to harness electrostatic catalysis in nitroxidemediated polymerization. Previously we had shown that the electric field from remote negatively charged functional groups could dramatically lower the bond dissocia-

a)

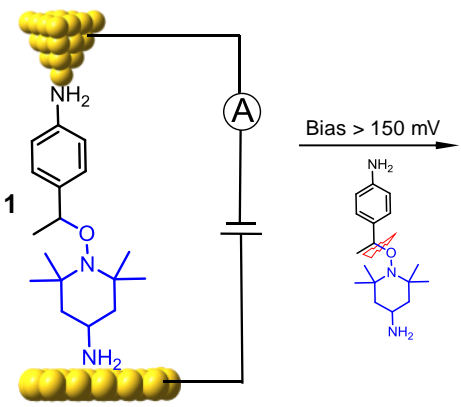

d)

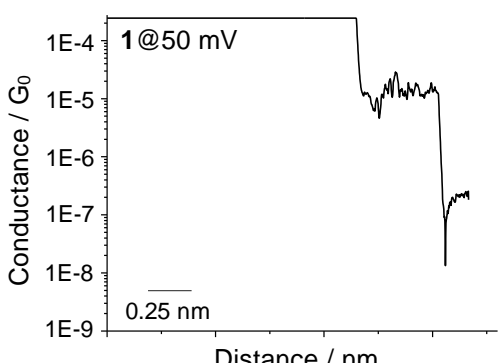

g)

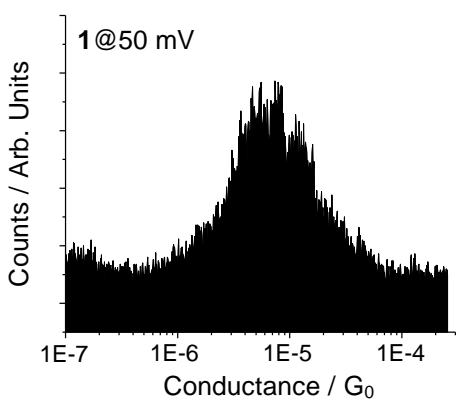

b)

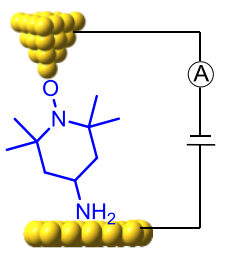

e)

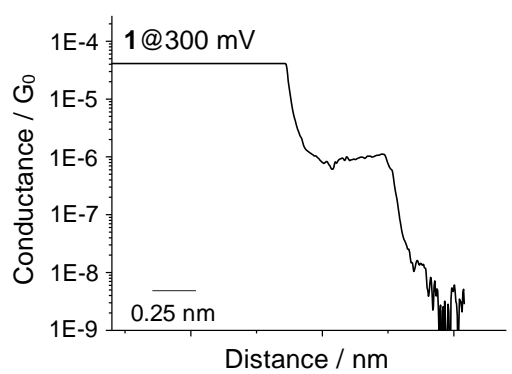

h)

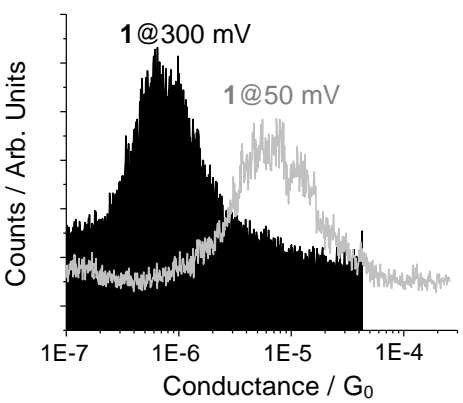

tion energy of alkoxyamines and facilitate their homolysis at temperatures lower than otherwise possible. ${ }^{12 a, 13}$ The catalytic effect is delivered by the charges of ionized functional groups and can therefore be predictably c)
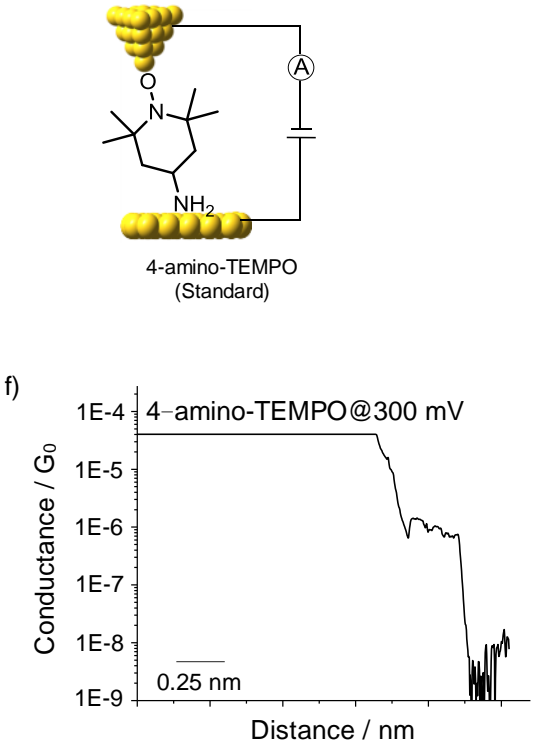

i)

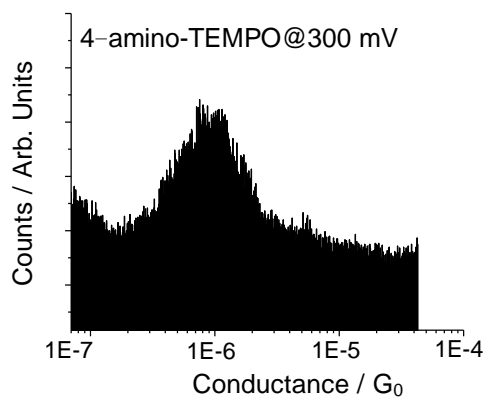

Figure 1. Electrostatic catalysis in the homolysis of alkoxyamines. (a-c) Schematic depiction of the STM-BJ setup for a single-molecule junction experiment used to investigate the effect of an external electrical field on the breaking of a C-ON bond. We used single-molecule STM-BJ conductance measurements to probe the fate of the alkoxyamine molecule 1 under a variable electric field stimulus in a low dielectric solvent. The experiments capture discrete electrical signals from either the intact parent molecule 1 or from the putative 4-amino-TEMPO fragment that is produced upon the homolysis of 1. A STM tip is brought into and out of contact with an $\mathrm{Au}(111)$ surface while this is covered with a diluted solution of the molecule of interest (either 1 or a 4-amino-TEMPO standard in mesitylene/DCM, 10:1, v/v). The surface is biased against the tip and the current versus distance signal is collected as the tip is moved away from the surface. (d-f) Typical current versus distance traces with conductance plateaus indicative of a single-molecular junction. The current drops from the currentsaturation value to the current-amplifier detection limit, passing through breakage steps, "plateaus", each of a specific conductance value. ( $\mathrm{g}-\mathrm{i})$ Conductance histograms showing the electrical "fingerprints" of either the intact alkoxyamine 1 (i.e. before splitting, tip-surface bias $<150 \mathrm{mV})$ at $1 \mathrm{E}-5 \mathrm{G}_{0}\left(\mathrm{G}_{0}=\left(2 \mathrm{e}^{2} / h=77.5 \mu \mathrm{S}\right.\right.$, quantum of conductance) or the free nitroxide that is unmasked after splitting (bias $>150 \mathrm{mV}$ ) at $1 \mathrm{E}-6 \mathrm{G}_{0}$. The molecular conductance obtained upon homolysis of 1 , as shown in $(\mathrm{h}, 1 @ 300 \mathrm{mV})$, is a perfect match of the results from control experiments where the two electrodes are forming junctions in a standard sample of commercial 4-amino-TEMPO (c,f,i).

adjusted according to $\mathrm{pH} .{ }^{13 \mathrm{a}}$ The electric field that is associated with these charges alters the stability of chargeseparated resonance contributors $\left(\mathrm{N}-\mathrm{O}^{\circ} \leftrightarrow \mathrm{N}^{+}-\mathrm{O}^{-}\right)$and hence the extent of stabilization of the nitroxide. This ultimately leads to electrostatic catalysis of reactions involving such "switchable" nitroxides, including alkoxy- 
amine dissociation and hydroxyl amine hydrogen transfer. However, whilst this electrostatic effect is large in the gas phase, ${ }^{122}$ in solution it progressively diminishes as the polarity of the solvent increases. ${ }^{13 \mathrm{~b}}$ Further, whilst this type of $\mathrm{pH}$ switch is possible in solvents of low polarity, ${ }^{13 \mathrm{a}}$ its practical use here is hampered by the low solubility of charged species, leading to a trade-off between solubility

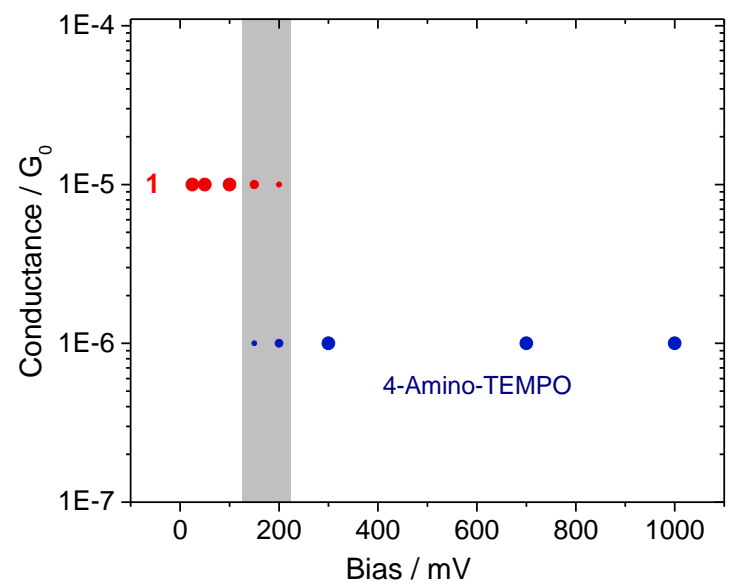

Figure 2. Electric fields and breaking probability of single-molecules. The plot shows the effect of the bias between the STM tip and the Au(111) surface over the fate of several thousand molecular junctions (ca. 4000 for each data point) each obtained by electrically "wiring" molecules of alkoxyamine $\mathbf{1}$ in a STM-BJ experiment. At biases in the range between 150 and $200 \mathrm{mV}$, discrete conductance plateaus at both $1 \mathrm{E}-5 \mathrm{G}_{0}$ and $1 \mathrm{E}-6 \mathrm{G}_{0}$ appear, reflecting the co-existence of both the parent molecule 1 and its homolysis product (4-amino-TEMPO). The area of each data point reflects the relative bias-dependent abundance of the species.

and magnitude of the electrostatic catalytic effect.

This raises the question as to whether there is a better and more general method of introducing charges and ensuring they align with reaction axes. One strategy that we have successfully used relies on electrification of molecules while these are held under bias in the nanoscale gap between metal electrodes of a scanning tunneling microscope-break junction (STM-BJ) set-up. STM-BJ is the only technique available to bring together single molecule reactants between nanoscale electrodes, it is unparalleled in its ability to deliver a large oriented electric field $(\mathrm{V} / \mathrm{nm})$ which is here tunable and can catalyze several thousands of chemical events over short periods of time. Using STM-BJ (in mesitylene as solvent) we have recently demonstrated a link between electrostatic forces and the rate of formation of a carbon-carbon covalent bond. ${ }^{3 b}$ However, it remains that while this technique can be key in an experimental scrutiny leading to proof-of-concept data, it is clearly not practical to process workable quantities of materials in STM-BJ.
A simpler approach, suitable for unimolecular reactions at least, is to attach a molecule to an electrode. Previously we have shown that nitroxides can be grafted onto highlydoped, i.e. metallic, $\mathrm{Si}(100)$ electrodes; ${ }^{14}$ in the present work we use a similar monolayer-chemistry method to graft alkoxyamines. In this way reactants can be aligned the double layer of electrochemical cells. Moreover, by tethering an alkoxyamine to an electrode we hoped to use nitroxide mediated polymerization to grow surfacetethered polymer with narrow molecular weight distributions in a manner that was both spatially and temporally controlled.

In what follows we first use STM-BJ to demonstrate that an external electric field triggers homolysis of alkoxyamines at room temperature. We then show that in an electrochemical cell at an appropriate voltage, alkoxyamines also undergo room temperature cleavage, irrespective of whether they are tethered to an electrode or not. However, we show that the latter process is actually an ECE (electrochemical-chemical-electrochemical) process whereby the alkoxyamine is first oxidised prior to rapid cleavage into a nitroxide and carbocation, followed by oxidation of the nitroxide. Interestingly, the chemical step itself is nonetheless driven by electrostatic effects, even when the alkoxyamine is not tethered to a surface suggesting self-alignment in the double layer is possible and that directional electrostatic effects are possible in solution and may be an important and often overlooked contributing factor in organic electrochemistry.

\section{RESULTS AND DISCUSSION}

Electrostatic Cleavage of Alkoxyamines in a SingleMolecule Scanning Tunneling Microscope BreakJunction Experiment. To establish whether electrostatic effects can trigger alkoxyamine homolysis we conducted STM-BJ experiments. The repetitive formation of molecular junctions in the STM-BJ technique is an established method of accessing conductance measurements on single-molecules. ${ }^{15}$ It also allows access to a statistically significant pool of data on bond breaking (and/or reformation $^{3 \mathrm{~b}}$ ) in response to changes in field magnitude. We have explored the role of electric fields on the lysis of alkoxyamines by bridging a molecule (alkoxyamine 1, Figure 1a) between a gold STM tip and an Au(111) substrate under a bias stimuli of variable magnitude. Alkoxyamine $\mathbf{1}$ is symmetrically substituted with primary amino groups at opposite ends of the molecule (see Supporting Sections $S_{1}$ and $S_{2}$ for synthetic procedures). These two primary amine pendants have affinity toward gold, allowing electrical contacts to be made with the reactant, i.e. molecules of $\mathbf{1}$, by both the STM Au tip and the Au(111) surface. The single molecule conductance is measured by repeatedly forming and breaking the molecular junction by "tapping" the STM tip in and out of contact with the $\mathrm{Au}(111)$ surface while this is covered by a diluted sample of 1 (in mesitylene/DCM, 10:1, v/v solvent). Current plateaus appear in the pulling curves reflecting the formation of a 
single-molecule junction. By means of accumulating thousands of individual current-distance traces, (Figure 1d-f), we built conductance histograms (Figure 1g-i) showing peak maxima that reflect the most probable conductance value of the molecule(s) in the junction at a given tip-surface bias.

Figure 2 summarizes visually the outcome of STM-BJ experiments on 1. In brief, below a threshold bias of 150 $\mathrm{mV}$ the statistical analysis of individual plateaus in the current versus distance curves leads to a single prominent peak in the conductance histogram at $c a$. $1 \mathrm{E}-5 \mathrm{G}_{\mathrm{o}}$ (where $\mathrm{G}_{\mathrm{o}}=77.5 \mu \mathrm{S}$ ). This peak is unambiguously assigned to the parent alkoxyamine, indicating the presence exclusively of intact molecules of $\mathbf{1}$ in the electrified gap (left column in Figure 1). Between $100 \mathrm{mV}$ and $200 \mathrm{mV}$, however, a second plateau appears at $1 \mathrm{E}-6 \mathrm{G}_{\mathrm{o}}$. Finally, above $300 \mathrm{mV}$, the ${ }_{1} E-5 G_{0}$ peak disappears completely to be replaced by a $1 E-6 G_{0}$ electrical signature (Figure 2 and panels e and $h$ in Figure 1). This conductance signature at ${ }_{1} E-6 G_{0}$ is assigned to nitroxide species that are generated from the electric field-induced splitting of $\mathbf{1}$ by a bias $>150 \mathrm{mV}$. Nitroxides have a known affinity for gold surfaces, ${ }^{16}$ and the same $1 E-6 G_{0}$ conductivity signature is observed in control STM-BJ experiments that are performed using a standard 4-amino-TEMPO solution (right column in Figure 1). Moreover, unlike for the alkoxyamine 1, STM-BJ experiments performed only in the presence of 4-aminoTEMPO molecules do not show a dependency of molecular conductance on bias, and for instance the conductance $/ G_{0}$ ratio of these controls is unchanged between 50 $\mathrm{mV}$ and $300 \mathrm{mV}$ (see panels c-d in Supporting Figure $\mathrm{S} 1$ ). It appears therefore extremely unlikely that the ${ }_{1} E-6 G_{0}$ signature in this field-assisted lysis of $\mathbf{1}$ is arising from chemical entities other than a free nitroxide fragment. For example, STM-BJ control experiments of 4-vinylaniline (i.e. a putative end product of the homolysis ${ }^{17}$ ) are shown in panels e-f of Supporting Figure $\mathrm{S}_{1}$ and carry no evidence of any sizable peak in conductance histogram. The STM tip-to-substrate bias appears therefore to guide the redistribution between an alkoxyamine-only population (up to ca $100 \mathrm{mV}$ of dc bias between STM tip and substrate) to a mixed alkoxyamine/nitroxide population (between 150 and $200 \mathrm{mV}$ ) and ultimately to a nitroxide-only presence (bias over $300 \mathrm{mV}$ ).

The role of the electric field in promoting the homolysis of the alkoxyamine can be explained by a theoretical model of the system. Quantum-chemical calculations of the reaction profile in the presence of a field of varying strength that is aligned along the $\mathrm{N}-\mathrm{O}$ bond axis suggest that the homolysis of $\mathbf{1}$ is possibly promoted by as much as $35 \mathrm{~kJ} \mathrm{~mol}^{-1}$. This barrier-lowering effect is consistent with the expected stabilization of the charge-separated resonance contributor to the nitroxide radical $\left(\mathrm{N}-\mathrm{O}^{\circ} \leftrightarrow\right.$ $\mathrm{N}^{+}-\mathrm{O}^{-}$) and is enough to account for the radical formation. Electrostatic effects can thus promote homolysis of alkoxyamines and can explain the observed STM re- sults. However, it is difficult to extract truly quantitative theoretical data on the field/barrier relationship. This is because, while we clearly can control in STM-BJ the alkoxyamine/nitroxide population, the upper limit of the experimental electric field $(\mathrm{V} / \mathrm{nm})$ in the STM experiment is nonetheless relatively difficult to quantify as the distance between the tip and the $\mathrm{Au}(111)$ plate varies during the tapping experiments. Therefore, as a conservative measure, the range of field strengths considered in the theoretical model are probably a lower estimate (Supporting Section $\mathrm{S}_{7}$ ).

Electrochemical Cleavage. While the STM experiments provide proof of concept that electric fields can trigger alkoxyamine homolysis, they do not offer a practical route to chemical synthesis. So, we next explored whether electrostatic effects could instead be harnessed in an electrochemical cell. Chemists appreciate that the condition of bulk electro-neutrality is not valid at interfaces, and strong field gradients are ubiquitous at electrode/electrolyte interfaces (electric double layers). ${ }^{4 a, 18}$ Anodization of a surface-tethered alkoxyamine $\mathbf{2}$ in a perchlorate-based electrolyte (S-1 surface construct in Figure 3, surface modification and XPS/XRR characterizations in Supporting Scheme $\mathrm{S}_{2}$ and Supporting Figures $\mathrm{S}_{2}$ and $S_{3}$, respectively) shows the gradual appearance of a TEMPO redox signature ${ }^{14,19}$ upon positive biases. A similar electrochemical pre-treatment with negative bias has only a minor effect on the cleavage. Under these conditions, the greater yield with positive bias was unexpected as the alkoxyamine (and product nitroxide radical) is tethered with the nitrogen-side of the polar $\mathrm{N}^{\delta+}-\mathrm{O}^{\cdot \delta-}$ bond closest to the electrode, 

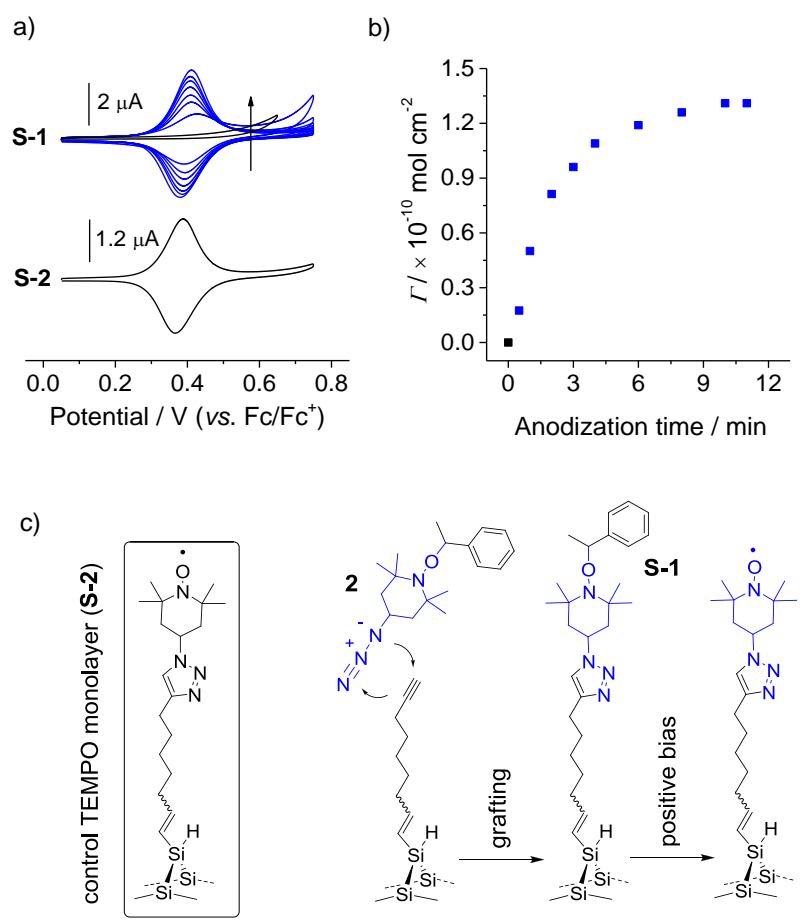

Figure 3. Anodic cleavage of a surface-tethered alkoxyamine (S-1) and its conversion into a $\mathrm{Si}(100)$ nitroxideterminated monolayer (electrolyte is $1.0 \times 10^{-1} \mathrm{M} \mathrm{Bu}_{4} \mathrm{NClO}_{4}$ in $\mathrm{MeCN}$ ). The monolayer distal-end of $\mathbf{S - 1}$ samples is the phenylethyl portion of an alkoxyamine molecule (2) and it is lost to the electrolyte upon electrolysis. (a) Cyclic voltammograms $\left(100 \mathrm{mV} \mathrm{s}^{-1}\right)$ acquired before (black trace) and after (blue trace) applying a positive bias to S-1 samples prepared on $\mathrm{Si}(100)$ electrodes. The potential is stepped from open circuit to $0.65 \mathrm{~V}$ ( $\mathrm{vs}$. $\mathrm{Fc} / \mathrm{Fc}^{+}$) for a $30 \mathrm{~s}$ period before recording a voltammogram and selected traces are presented in figure (blue traces). Anodization of S-1 results in the progressive appearance of a redox signature that is in good agreement with that of a surface-tethered TEMPO control (S-2, black trace). The TEMPO coverage rises in increments of about $2.6 \times 10^{-11} \mathrm{~mol} \mathrm{~cm}^{-2}$, and reaches a maximum of ca. $1.31 \times 10^{-10} \mathrm{~mol} \mathrm{~cm}^{-2}$, equivalent to ca. $25 \%$ of a close-packed TEMPO monolayer assembled on a gold surface. ${ }^{20}$ (b) Plot of the electrochemically-determined changes to the surface coverage of redox-active nitroxide radicals as a function of the anodization time of S-1 samples. (c) Reaction schematics for the alkoxyamine surface model (S-1) and controls (S-2)

hence we had initially assumed that a negative electrode would better stabilize the nitroxide radical product. However, follow up diffusive experiments revealed that the situation is much more complicated than we first anticipated (i.e. simple stabilization of charge-separated contributors by distal negative charges) and that in electrolytes the positive bias was actually leading to oxidation of the alkoxyamine. This in turn led to its
3
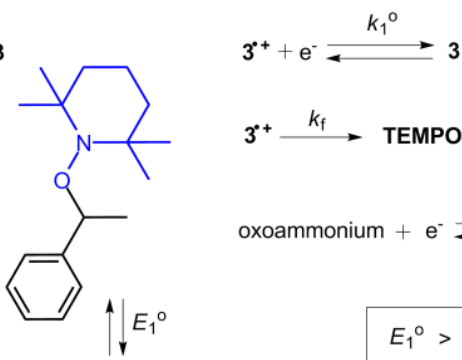

$(\overrightarrow{\mathrm{E}})$

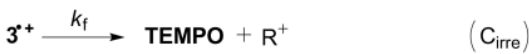

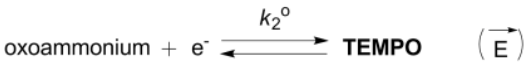

$E_{1}^{\circ}>E_{2}^{\circ}$
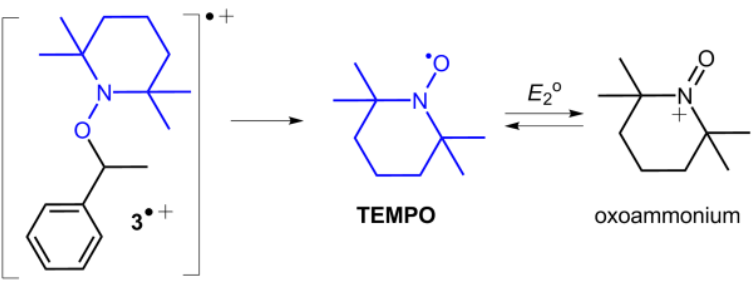

TEMPO

oxoammonium

Scheme 1. Consecutive $E C_{\text {irre }} E$ mechanism accounting for the anodic fragmentation of alkoxyamines. The anodic intermediate $\left(3^{++}\right)$is an unstable transient species which is found to undergo rapid unimolecular decomposition $\left(\mathrm{C}_{\text {irre }}, k_{f}\right.$ in the order $10^{6} \mathrm{~s}^{-1}$, details on individual electrolyte systems in the Supporting Information) releasing at room temperature the redox-active nitroxide fragment (TEMPO).

cleavage to a carbocation and nitroxide, followed by oxidation of the latter to oxoammonium (Scheme 1). We subsequently saw this same behavior in non-surface tethered nitroxide radicals, where digital simulations of voltammetry and in-situ electrochemical EPR experiments were able to help validate an $\mathrm{EC}_{\mathrm{irr}} \mathrm{E}$ mechanism and measure the thermodynamic and kinetic parameters of the process. Figure 4a and Supporting Figures $\mathrm{S}_{4}-\mathrm{S}_{7}$ show representative experimental voltammograms, currenttime transients (chronoamperometry) and digital simulations for the electrochemical oxidation of 3 at platinum electrodes in $\mathrm{MeCN} / \mathrm{Bu}_{4} \mathrm{NClO}_{4}$ solutions. Extensive data sets where the voltage sweep rate and concentration of the alkoxyamine reagent were varied over two orders of magnitude range, respectively, are in Supporting Figures S5-S6.

The first anodic segment is featureless, i.e. it displays only a capacitive-like current until the potential is expanded to about $0.7 \mathrm{~V}$, where the current raises rapidly to show a clear oxidative wave before the onset of background processes. This anodic process is irreversible over the time scale of the experiment. The lack of a back peak in cyclic voltammetry (Figure 4a), as well as the modelling of current-time transients (triple potential step chronoamperometry data in Figure $\mathrm{S}_{7}$ ), indicate the electrontransfer step is followed by a fast follow-up homogeneous chemical reaction. A satisfactory fit of both types of experiments does not require inclusion a second-order backward process in the chemical step, hence it is apparent that alkoxyamine 3 undergoes fragmentation after its 
initial electrochemical oxidation with a rather large chemical rate $\left(k_{f}=5.0 \times 10^{6} \mathrm{~s}^{-1}\right)$. The current magnitude of the most anodic wave as well as the appearance of a redox signature upon scan reversal both indicate that one of the two fragments is electrochemically-active. The electrode kinetics and thermodynamics for this fragment are a

a)

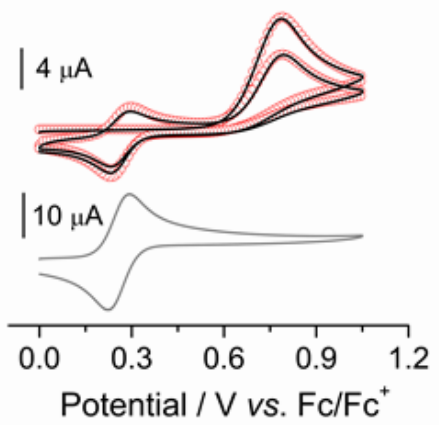

b)

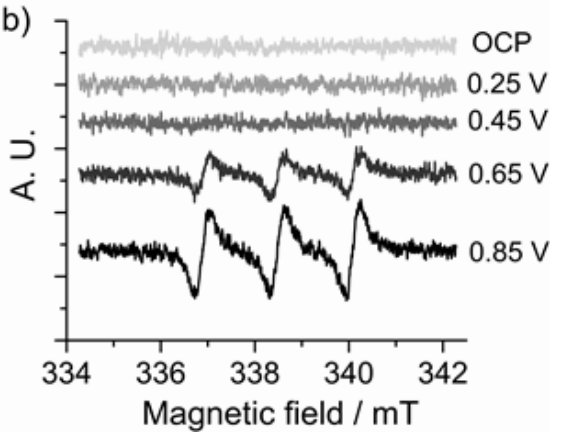

Figure 4. Anodic electrochemistry of alkoxyamines 3 at platinum electrodes. (a) Experimental (solid lines) and simulated (empty symbols) cyclic voltammograms of $0.5 \times 10^{-3} \mathrm{M}$ 3 , and experimental data (offset grey trace) of $0.5 \times 10^{-3} \mathrm{M}$ TEMPO controls. The best-fit parameters are $D\left(3 / 3^{\cdot+}\right)=7.8$ $\times 10^{-6} \mathrm{~cm}^{2} \mathrm{~s}^{-1}, D$ (TEMPO/oxoammonium) $=2.2 \times 10^{-5} \mathrm{~cm}^{2}$ $\mathrm{s}^{-1}, E_{1}^{0}=0.78 \mathrm{~V}, k_{1}^{0}=0.05 \mathrm{~cm} \mathrm{~s}^{-1}, E_{2}^{0}=0.195 \mathrm{~V}, k_{2}^{0}=0.08$ $\mathrm{cm} \mathrm{s} \mathrm{s}^{-1}, k_{f}=5.0 \times 10^{6} \mathrm{~s}^{-1}$. (Electrolyte is $1.0 \times 10^{-1} \mathrm{M}$ $\mathrm{MeCN} / \mathrm{Bu}_{4} \mathrm{NClO}_{4}$, and scan rate is $100 \mathrm{mV} \mathrm{s}{ }^{-1}, 0.7 \mathrm{~cm}^{2}$ platinum macrodisk electrode). (b) In-situ electrochemical EPR measurements conducted in $1.0 \times 10^{-1} \mathrm{M}$ $\mathrm{DCM} / \mathrm{Bu}_{4} \mathrm{NPF}_{6}$ in the presence of $0.5 \times 10^{-3} \mathrm{M}$ of 3 . The specified electrolysis bias (labels to curves vs. $\mathrm{Fc} / \mathrm{Fc}^{+}$) was imposed to a platinum wire electrode for $360 \mathrm{~s}$ with the EPR data being accumulated over the last $60 \mathrm{~s}$ of the potential step.

perfect match of those obtained for control experiments using TEMPO solutions $\left(E_{T E M P O}^{0}=0.21 \mathrm{~V} v s . \mathrm{Fc}_{\mathrm{C}} / \mathrm{Fc}^{+}, k_{T E M P O}^{0}\right.$ $=0.08 \mathrm{~cm} \mathrm{~s}^{-1}$, lower grey trace in Figure $4 \mathrm{a}$ and extensive data sets in Supporting Figures $\mathrm{S}_{4}$ and S8). These controls, in conjunction with in-situ electrochemical EPR data (Figure $4 \mathrm{~b}$ and Supporting Figure $\mathrm{S}^{21}$ ) indicate that lysis of the oxidized alkoxyamine $\left(3^{+}\right)$is most likely occurring on its $\mathrm{C}-\mathrm{ON}$ bond with release of the parent nitroxide (i.e. TEMPO, vide infra for the discussion on a quantum model of the system). In brief, digital simula- tions of the electrochemical data lead to the following main conclusions: i) the overall mechanism needs to be analyzed as an $\mathrm{EC}_{\mathrm{irre}} \mathrm{E}$ process (i.e. sequential electrochemical-chemical-electrochemical steps, Scheme 1); ii) a large cation radical decay reaction rate constant $\left(k_{f}\right)$ is needed to reproduce the shapes of all the experimental curves; iii) the anodic wave at ca. $0.7 \mathrm{~V}$ includes two oneelectron processes, the oxidation of 3 (refined $E_{1}^{0}=0.78 \mathrm{~V}$ ) and the oxidation of a portion of the newly-formed TEMPO units (refined $E_{2}^{0}=0.195 \mathrm{~V}$ ). In brief, after the oxidation of the parent alkoxyamine, $3^{\cdot+}$ participates in a fast homogeneous chemical reaction explaining the mechanistic framework that leads to a free nitroxide and a carbocation at the electrified interface $\left(3^{*} \rightleftarrows\right.$ TEMPO + $\left.\mathrm{R}^{+}, k_{\mathrm{b}} \rightarrow \mathrm{o}\right)$.

Electrostatic Catalysis? The experimental finding that alkoxyamines, normally stable at room temperature, undergo rapid and irreversible room temperature homolysis upon oxidation is remarkable when analyzed in terms of the expected energetics of the process. High-level $a b$ initio calculations were conducted at a level of theory previously demonstrated to reproduce experimental redox potentials of nitroxides ${ }^{22}$ and homolysis equilibrium constants of neutral alkoxyamines ${ }^{23}$. The computed oxidation potentials versus $\mathrm{Fc} / \mathrm{FC}^{+}$of $3(0.83 \mathrm{~V})$ and TEMPO (o.20 V) in this work are also in excellent agreement with the corresponding best-fit experimental values $\left(E_{1}^{0}=0.78 \mathrm{~V}\right.$ and $\left.E_{2}^{0}=0.195 \mathrm{~V}\right)$. Moreover, the calculations predict the correct preferred fragmentation pattern (i.e. to a nitroxide and carbocation rather than an oxoammonium cation and a carbon centered radical) further validating their accuracy. However, when the energetics of the homolysis of the oxidized alkoxyamine is calculated in $\mathrm{MeCN}$ it is unfavorable by $35 \mathrm{~kJ} \mathrm{~mol}^{-1}$, a finding that is inconsistent with the rapid decomposition observed experimentally.

Importantly, the calculations only take account of the solvent and fail to consider the electric fields experienced in the near-surface double-layer. When these were taken into account, it is then clear that the homolysis is promoted (Figure 5). For instance, by using only a simple background electric field of $0.5 \mathrm{~V} \mathrm{~nm}^{-1}$, a value which reflects the potential gradient that is most likely experienced by species reaching the electrified interface, ${ }^{24}$ the homolysis energies were lowered but not by enough to explain the experimentally observed reaction rates. However, combination of a static electric field and a continuum solvent model (in which the solvent is itself modelled as an electric field) is likely to provide a rather crude approximation of the true environment. Unfortunately modelling a full ensemble of explicit ions and solvent molecules while maintaining a suitably high level of theory is impractical. However, we can show that when either an explicit ion or an explicit solvent molecule is included in the calculations, further lowering is observed (Figure 5). Hence it is most likely that the electrostatic environment within the double-layer is helping to promote homolysis of a compound that would otherwise be stable. 
While the calculations suggest that the environment in the double layer is promoting homolysis, it is difficult to establish whether this is due to explicit bonding interactions or electrostatics as full ensemble of species cannot be modelled accurately (vide supra). To examine whether the effects were primarily electrostatic, experiments were thus conducted with anions that are significantly less coordinating than perchlorate, such as hexafluorophosphate and tetrakis[3,5-bis(trifluoromethyl)phenyl]borate (BARF hereafter) (see Supporting Figures Sio-S14). The results are in line with the observations in the perchlorate system, pointing to an $\mathrm{EC}_{\mathrm{irr}} \mathrm{E}$ mechanism with the backward chemical reaction not being operative in the time scale of the experiments. For example, with scan rates that varied over three orders of magnitude (from $75 \mathrm{mV} \mathrm{s}^{-1}$ to $8 \mathrm{~V} \mathrm{~s}^{-1}$, Supporting Figure $\left.\mathrm{S}_{13}\right)$ the refinement of the voltammograms of alkoxyamine 3 in BARF electrolytes $\left(k_{f}\right.$ $\left.=5.2 \times 10^{6} \mathrm{~s}^{-1}\right)$

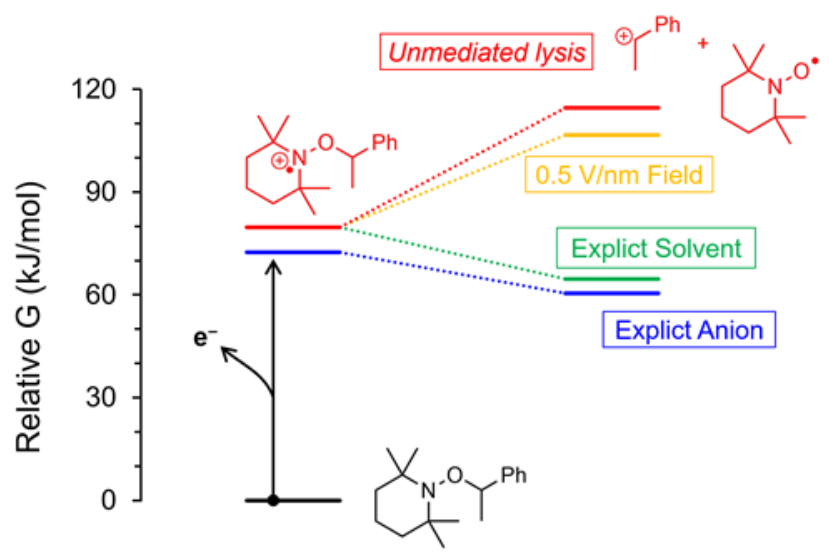

Figure 5. Theoretical potential energy surface for the oxidative cleavage of alkoxyamine 3 in MeCN. The homolysis of the unperturbed ('free') radical-cation (red pathway) is strongly thermodynamically disfavoured. However, homolysis can be made more favourable by a static electric field, and by interactions with an explicit anion and/or with an explicit solvent molecule.

suggest kinetic, as well as mass transport, parameters that are a close match of those obtained in $\mathrm{Bu}_{4} \mathrm{NClO}_{4}$ electrolytes $\left(k_{f}=5.0 \times 10^{6} \mathrm{~s}^{-1}\right)$ (Figures $\left.\mathrm{S}_{5}\right)$. Within the same solvent, the results remained unchanged (see Supporting Figure $\mathrm{S}_{5}$, S10 and $\mathrm{S}_{13}$ ), however, there were small differences between solvents (MeCN versus DCM, see Supporting Figure $\mathrm{S}_{5}$ and Figure $\left.\mathrm{S}_{15}\right)$. Indeed, this was also backed by theoretical calculations (Supporting Figure S19). Collectively, the experiments suggest that it is primarily the electrostatic environment in the double layer driving cleavage rather than explicit covalent interactions with the electrolyte ions.

\section{CONCLUSION}

Two areas of chemistry that are attracting increasing attention at present are the use of electrochemical reactions in organic synthesis, so-called synthetic organic electrochemistry, ${ }^{25}$ and the use of static electric fields to catalyse non-electrochemical reactions, so-called electrostatic catalysis. ${ }^{1-3,12 a, 13}$ The present work sits at the intersection of these fields. We have explored the prospect of using electricity and electrostatics as alternative triggers of alkoxyamine decomposition. In STM-BJ experiments we have shown that static electric fields can trigger alkoxyamine dissociation to nitroxides and carbon-centred radicals under conditions where they would otherwise be stable, while in the electrochemical experiments we have demonstrated an alternative means of homolysis, albeit to nitroxides and carbocations instead. We show that this latter reaction, an electrochemical $\mathrm{EC}_{\mathrm{irr}} \mathrm{E}$ process, proceeds for free alkoxyamines in solution as well as for alkoxyamines tethered to a silicon electrode, and can provide a convenient strategy for in situ generation of nitroxides and carbocations. Importantly, we show that even in the electrochemical process the $\mathrm{C}-\mathrm{ON}$ cleavage step itself is only made possible by electrostatic effects, highlighting their previously overlooked role in organic electrochemistry.

\section{EXPERIMENTAL SECTION}

STM-BJ Measurement. Measurements of singlemolecule conductivity using the STM-BJ technique were carried out using PicoSPM I microscope controlled by a Picoscan-2500 electronics (all from Agilent Technologies) and using a custom PTFE-STM cell for solid/liquid samples. The current versus distance curves (gold STM tip-to$\mathrm{Au}(111)$ substrate distance) were captured using a NIDAQmx/BNC-211o National Instruments (LabVIEW) and analyzed with a LabVIEW code. The procedure of breakjunction experiment is based on moving the STM tip to tunneling distance over an $\mathrm{Au}(111)$ surface that is covered with a dilute sample containing the molecules of interest (alkoxyamine 1, 4-amino-TEMPO and 4-vinylaniline in mesitylene /DCM, 10:1, v/v). The STM current-feedback is then turned off and the tip is driven into and out of contact with the surface at a speed of ca. $50 \mathrm{~nm} / \mathrm{s}$. This 2points cycle is repeated thousands of times enabling capturing of ca. 4000 distance versus current curves (current decays) for each set of data. Plateaus, indicative of the formation and subsequent rapture of single-molecule electrical junctions, appear during the pulling cycle of the externally driven 2 points loop. The current decays are then accumulated in conductance histograms. The peak maximum in the conductance histogram resemble the single-molecule conductivity of the sample (conductance (G) was calculated using the equation $\mathrm{G}=I_{\text {step }} / V_{\text {bias }}$, where $I_{\text {step }}$ is the plateau current and $V_{\text {bias }}$ is the voltage difference between the tip and surface).

Electrochemical Methods and Digital Simulations. Electrochemical experiments were carried out using a $\mathrm{CH}$ $650 \mathrm{D}$ electrochemical analyzer ( $\mathrm{CH}$ Instruments, Austin, 
TX) and a single compartment three-electrode PTFE cell. Platinum disks or chemically-modified $\mathrm{Si}(100)$ surfaces (S$\mathbf{1}$ and $\mathbf{S}-\mathbf{2}$ in Figure 3 and Supporting Scheme S2) served as the working electrode (analysis of diffusive systems of alkoxyamine 3 or surface-tethered models using alkoxyamine 2, Scheme 1 and Figure 3, respectively), a platinum coil was used as counter electrode, and a plastic body silver/silver chloride "leakless" as the reference electrode (eDAQ, part ETo72-1). The active area of the platinum disk was electrochemically-determined prior to each experiment from the refinement of a $\mathrm{E}$ model against experimental voltammogramms measured in $1.0 \times 10^{-1} \mathrm{M}$ $\mathrm{MeCN} / \mathrm{Bu}_{4} \mathrm{NClO}_{4}$ in the presence of $0.5 \times 10^{-3} \mathrm{M} \mathrm{mM}$ ferrocene ( $\mathrm{Fc}$ in shorthand hereafter). The geometric area of the $\mathrm{Si}(100)$ working electrode was defined to $28 \mathrm{~mm}^{2}$ by a Viton $^{\circledast}$ gasket of rectilinear cross-section. The backside of the silicon sample was scratched with emery paper and rubbed with gallium-indium eutectic. A planar copper plate was pressed against the sample backside and served as ohmic contact. The reference electrode was calibrated before and after each experiment against the apparent formal potential of the ferricenium/ferrocene couple $\left(\mathrm{Fc}^{+} / \mathrm{Fc}\right)$ at a platinum disk electrode in $0.5 \times 10^{-3} \mathrm{M}$ Fc. All potentials are reported against the $\mathrm{Fc}^{+} / \mathrm{Fc}$ couple. Electrochemical experiments were performed at room temperature $\left(23 \pm 2{ }^{\circ} \mathrm{C}\right)$ in a grounded Faraday cage under argon. In-situ electrochemical EPR spectra were acquired in a cylindrical cell equipped with a platinum wire as a working electrode $(2.5 \mathrm{~cm}$ length and $0.2 \mathrm{~mm}$ diameter), a platinum coil as an auxiliary electrode and a silver wire as a pseudo-reference electrode. The experiments were conducted using a JEOL JES-FA 200, X-band CW-EPR spectrometer operating at $100 \mathrm{kHz}$ field modulation coupled with the Ecochemie AUTOLAB Autolab PGSTAT302N+BA potentiostat-galvanostat. During measurement the microwave power was equal to $0.995 \mathrm{~mW}$ and modulation width was equal to $0.1 \mathrm{mT}$ and the influence of modulation width on registered spectra was also verified. Digital simulations of cyclic voltammetry and chronoamperometry experiments were performed in DigiElch-Professional v7 (ElchSoft). Simulated kinetic, thermodynamic and transport parameters for the electrode reaction were determined from fittings of experimental data sets that covered a range of concentrations assuming an $\mathrm{EC}_{\text {irre }} \mathrm{E}$ mechanism. Butler-Volmer kinetics was used to estimate charge transfer parameters. A semi-infinite $1 \mathrm{D}$ diffusion was assumed at both macrodisk planar and cylindrical electrodes (chronoamperometry in EPR experiments). For microdisks we considered semi-infinite $2 \mathrm{D}$ diffusion fronts with $27(\mathrm{X})$ and $23(\mathrm{Y})$ grid points. The cell iR drop was not compensated during measurement. Values of cell resistance were measured by electrochemical impedance spectroscopy and used in the simulations (data of resistance are: $140 \Omega$ for $\mathrm{MeCN} / \mathrm{Bu}_{4} \mathrm{NPF}_{6}, 144 \Omega$ for $\mathrm{MeCN} / \mathrm{Bu}_{4} \mathrm{NClO}_{4}, 1466 \Omega$ for $\mathrm{MeCN} / \mathrm{NaBARF}, 1220 \Omega$ for $\mathrm{DCM} / \mathrm{Bu}_{4} \mathrm{NPF}_{6}$, with the exception of the borate salt, which is present at $1.0 \times 10^{-2} \mathrm{M}$, all supporting salts are at a concentration of $\left.1.0 \times 10^{-1} \mathrm{M}\right)$. Adsorption steps were neglected in the simulations and the transfer coefficients for both electrons transfer reactions (TEMPO/oxoammonium and $3 / 3^{\circ+}$ ) were assumed as constants $(\alpha, 0.5)$ for fitting purposes. Including a DISPCOMP pathway ${ }^{26}$ does not improve the results. In the simulation the homogeneous chemical step for the fate of the putative benzylic cation fragment $\left(\mathrm{R}^{+}\right)$was not considered as this has no effect on the quality of the fits. The diffusion coefficients of 3 and $3^{*+}$ were obtained from the best-fits of an $\mathrm{EC}_{\text {irre }} \mathrm{E}$ model of the linear sweep voltammogram at platinum microdisk electrodes and were assumed equal. For the chemical step, only the forward $\left(k_{f}\right)$ constant is considered. The second-order backward $\left(k_{b}\right)$ constant tends to zero (i.e. backward chemical reaction is not operative in the time scale of the experiments). Although the values of the formal potentials and rate constants obtained from the fitting of the different experimental data show some differences (non-significant in the case of the rate constants) likely reflecting deviations with respect to the assumptions of the theoretical modelling (specifically, linear diffusion conditions and equal diffusion coefficients), overall the consistency between the different sets of independent experiments is acceptable. Moreover, in the case of the formal potentials the experimental values are further supported by their good agreement with computational calculations.

Computational Methods. Full details of all theoretical procedures use for simulating both the STM experiments and electrochemical experiments are provided in the Supporting Information, sections $\mathrm{S}_{7}$ and S8 respectively.

For the STM experiments, the principle aims were to study the effect of external electric field (EEF) on the reaction energies, rather than assess absolute reaction energies. For this purpose the Mo6-2X/6-31+G(d) as the level of theory, which has been benchmarked and used in previous studies of EEFs, ${ }^{3 \mathrm{~b}}$ was sufficient. Full conformer searching and geometry relaxation of each species was performed and the lowest energy conformer then used for subsequent calculations involving fields. Solvation energies in mesitylene solvent were calculated with the SMD solvent model, and all calculations were performed in Gaussian 09. ${ }^{27}$

For the electrochemical experiments, accurate absolute values of the oxidation potentials and homolysis energies were required and so higher levels of theory were used, based on extensive benchmarking studies, including for alkoxyamine homolysis energies, ${ }^{23}$ and nitroxide oxidation potentials and ion-pairing energies. ${ }^{14,22}$ All geometry optimizations and frequency calculations were performed at the Mo6-2X/31+G(d,p) level of theory. Entropies, thermal corrections and zero-point vibrational energies were scaled by recommend scale factors. ${ }^{28}$ Improved singlepoint energies were calculated using the high-level composite $a b$ initio $\mathrm{G}_{3}\left(\mathrm{MP}_{2}, \mathrm{CC}\right)(+)$ method, a variation of standard $\mathrm{G}_{3}\left(\mathrm{MP}_{2}, \mathrm{CC}\right)^{29}$ where calculations with the 6${ }_{31} \mathrm{G}(\mathrm{d})$ basis set are replaced with corresponding 6- 
$31+G(d)$. These high-level calculations where utilized in conjunction with the ONIOM approximation ${ }^{30}$ for larger systems, with either standard $\mathrm{G}_{3}\left(\mathrm{MP}_{2}, \mathrm{CC}\right)$ or $\mathrm{UMP}_{2}$ used to model remote substituents effects. The solvation model based on density (SMD) ${ }^{31}$ was used to relax gas-phase structures to the solution-phase (at the UMo6-2X/6$31+G(d, p)$ level of theory). Free energies of solvation were then calculated on solution-phase geometries using the COSMO-RS model,,$^{32}$ using the ADF package, ${ }^{33}$ at the $\mathrm{BP} / \mathrm{TZP}$ level of theory (as it was parameterized for), and the remaining parameters were kept as default values ${ }^{34}$. All standard $a b$ initio molecular orbital theory, density functional theory (DFT) calculations were carried out using Gaussian $09^{27}$ and Molpro $2015^{35}$ software packages.

\section{ASSOCIATED CONTENT}

\section{Supporting Information}

The Supporting Information is avaialble free of charge on the ACS Publications website at http://pub.acs.org.

Materials and methods, synthesis and characterization of molecules and chemically-modified S(100) surfaces, XPS and XRR spectra data, STM-BJ methods and additional data, extensive electrochemical data and simulations for diffusive systems, additional theoretical methods and results.

\section{AUTHOR INFORMATION}

\section{Corresponding Authors}

josquin@um.es

michelle.coote@anu.edu.au

nadim.darwish@curtin.edu.au

simone.ciampi@curtin.edu.au

\section{ACKNOWLEDGMENTS}

This work was supported by grants from the Australian Research Council (ARC, DE160100732 (S.C.), DE160101101 (N.D.), CE 140100012 (L.Z., B.B.N., M.L.C. and G.G.W), FL11010o196 (G.G.W.)). J.G.S. and E.L. greatly appreciate the financial support provided by the Fundación Séneca de la Región de Murcia (Projects $19887 /$ GERM/15 and $18968 / \mathrm{JLI} / 13$ ) and by the Ministerio de Economía y Competitividad (projects CTQ2015-65243-P and CTQ-2015-71955-REDT). L.Z. would like to thank AINSE Ltd. for providing financial assistance. M.L.C. gratefully acknowledges allocations of supercomputing time on the National Facility of the Australian National Computational Infrastructure.

\section{REFERENCES}

(1) Shaik, S.; Mandal, D.; Ramanan, R. Nat. Chem. 2016, 8, 1091-1098.

(2) (a) Warshel, A. J. Biol. Chem. 1998, 273, 27035-27038; (b) Shaik, S.; Shurki, A. Angew. Chem. Int. Ed. 1999, 38, 586-625; (c) Sola, M.; Lledos, A.; Duran, M.; Bertran, J.; Abboud, J. L. M. J. Am. Chem. Soc. 1991, 113, 2873-2879.

(3) (a) Fried, S. D.; Bagchi, S.; Boxer, S. G. Science 2014, 346, 1510-1514; (b) Aragonès, A. C.; Haworth, N. L.; Darwish, N.; Ciampi, S.; Bloomfield, N. J.; Wallace, G. G.; Diez-Perez, I.; Coote, M. L.
Nature 2016, 531, 88-91; (c) Geng, C.; Li, J.; Weiske, T.; Schlangen, M.; Shaik, S.; Schwarz, H. J. Am. Chem. Soc. 2017, 139, 1684-1689.

(4) (a) Parsegian, A. Nature 1969, 221, 844-846; (b) Warshel, A.; Levitt, M. J. Mol. Biol. 1976, 103, 227-249; (c) Novotny, J.; Sharp, K. Prog. Biophys. Mol. Bio. 1992, 58, 203-224; (d) Fried, S. D.; Bagchi, S.; Boxer, S. G. J. Am. Chem. Soc. 2013, 135, 11181-11192.

(5) Simon, M. L. A.; Platre, M. P.; Marquèsbueno, M. M.; Armengot, L.; Stanislas, T.; Bayle, V.; Caillaud, M.; Jaillais, Y. Nat. Plants 2016, 2, 16089-16123.

(6) Stein, D.; Kruithof, M.; Dekker, C. Phys. Rev. Lett. 2oo4, 93, 035901-035904.

(7) Feng, J.; Graf, M.; Liu, K.; Ovchinnikov, D.; Dumcenco, D.; Heiranian, M.; Nandigana, V.; Aluru, N. R.; Kis, A.; Radenovic, A. Nature 2016, 536, 197-200.

(8) Pocker, Y.; Buchholz, R. F. J. Am. Chem. Soc. 1970, 92, 2075-2084.

(9) Warshel, A. Acc. Chem. Res. 1981, 14, 284-29o.

(10) Shaik, S. J. Am. Chem. Soc. 1981, 103, 3692-3701.

(11) Shaik, S.; Danovich, D.; Wu, W.; Hiberty, P. C. Nat. Chem. 2009, $1,443-449$.

(12) (a) Gryn'ova, G.; Marshall, D. L.; Blanksby, S. J.; Coote, M. L. Nat. Chem. 2013, 5, 474-481; (b) Gorin, C. F.; Beh, E. S.; Kanan, M. W. J. Am. Chem. Soc. 2011, 134, 186-189; (c) Akamatsu, M.; Sakai, N.; Matile, S. J. Am. Chem. Soc. 2017, 139, 6558-6561.

(13) (a) Klinska, M.; Smith, L. M.; Gryn'ova, G.; Banwell, M. G.; Coote, M. L. Chem. Sci. 2015, 6, 5623-5627; (b) Gryn'ova, G.; Coote, M. L. J. Am. Chem. Soc. 2013, 135, 15392-15403; (c) Gryn'Ova, G.; Smith, L. M.; Coote, M. L. Phys. Chem. Chem. Phys. 2017, 19, 2267822683.

(14) Zhang, L.; Vogel, Y. B.; Noble, B. B.; Gonçales, V. R.; Darwish, N.; Brun, A. L.; Gooding, J. J.; Wallace, G. G.; Coote, M. L.; Ciampi, S. J. Am. Chem. Soc. 2016, 138, 9611-9619.

(15) (a) Xu, B.; Tao, N. J. Science 2003, 301, 1221-1223; (b) Chen, F.; Hihath, J.; Huang, Z.; Li, X.; Tao, N. Annu. Rev. Phys. Chem. 2007, 58, 535-564; (c) Tao, N. J. Nat. Nanotechnol. 2006, 1, 173-181.

(16) (a) Krukowski, P.; Kowalczyk, P. J.; Krzyczmonik, P.; Olejniczak, W.; Klusek, Z.; Puchalski, M.; Gwozdzinski, K. Appl. Surf. Sci. 2009, 255, 3946-3952; (b) Swiech, O.; Hrynkiewicz-Sudnik, N.; Palys, B.; Kaim, A.; Bilewicz, R. J. Phys, Chem. C 2011, 115, 7347-7354.

(17) (a) Tsuchimoto, T.; Tobita, K.; Hiyama, T.; Fukuzawa, S.-i. J. Org. Chem. 1997, 62, 6997-7005; (b) Noji, M.; Ohno, T.; Fuji, K.; Futaba, N.; Tajima, H.; Ishii, K. J. Org. Chem. 2003, 68, 9340-9347.

(18) Hurth, C.; Li, C.; Bard, A. J. J. Phys. Chem. C 2007, 111, $4620-4627$.

(19) (a) Alévêque, O.; Seladji, F.; Gautier, C.; Dias, M.; Breton, T.; Levillain, E. ChemPhysChem 2009, 10, 2401-2404; (b) Alévêque, O.; Blanchard, P.-Y.; Breton, T.; Dias, M.; Gautier, C.; Levillain, E.; Seladji, F. Electrochem. Commun. 20o9, 11, 1776-1780.

(20) Blanchard, P.-Y.; Alévêque, O.; Breton, T.; Levillain, E. Langmuir 2012, 28, 13741-13745.

(21) Supporting Figure 12 shows the simulated TEMPO concentration profiles and cumulative number of TEMPO molecules across the EPR cell obtained upon the anodization of 3.

(22) Gryn'ova, G.; Barakat, J. M.; Blinco, J. P.; Bottle, S. E.; Coote, M. L. Chem-Eur. J. 2012, 18, 7582-7593.

(23) Hodgson, J. L.; Lin, C. Y.; Coote, M. L.; Marque, S. R. A.; Matyjaszewski, K. Macromolecules 2010, 43, 3728-3743.

(24) Yoon, Y. H.; Woo, D. H.; Shin, T.; Chung, T. D.; Kang, H. J. Phys, Chem. C 2011, 115, 17384-17391.

(25) (a) Yan, M.; Kawamata, Y.; Baran, P. S. Angew. Chem. Int. Ed. Engl. 2017, DOI: 10.1002/anie.201707584; (b) Yan, M.; Kawamata, Y.; Baran, P. S. Chem. Rev. 2017, 117, 13230-13319.

(26) Molina, Á.; Laborda, E.; Gómez-Gil, J. M.; Martínez-Ortiz, F.; Compton, R. G. Electrochim. Acta 2016, 195, 230-245.

(27) Frisch, J.; Trucks, G. W.; Schegel, H. B.; Scuseria, G. E.; Robb, M. A.; Cheeseman, J. R.; Scalmani, G.; Barone, V.; Mennucci, B.; Petersson, G. A.; Nakatsuji, H.; Caricato, M.; Li, X.; Hratchian, H. P.; Izmaylov, A.; Bloino, J.; Zheng, G.; Sonnenberg, J. L.; Hada, M.; 
Ehara, M.; Toyota, K.; Fukuda, R.; Hasegawa, J.; Ishida, M.; Nakajima, N.; Honda, Y.; Kitao, O.; Nakai, H.; Vreven, T.; Montgomery, J. A.; Jr. Peralta, J. E.; Ogliaro, F.; Bearpark, M.; Heyd, J. J.; Brothers, E.; Kudin, K. N.; Staroverov, V. N.; Keith, T.; Kobayashi, R.; Normand, J.; Raghavachari, K.; Rendell, A.; Burant, J. C.; Iyengar, S. S.; Tomasi, J.; Cossi, M.; Rega, N.; Millam, J. M.; Klene, M.; Knox, J. E.; Cross, J. B.; Bakken, V.; Adamo, C.; Jaramillo, J.; Gomperts, R.; Stratmann, R. E.; Yazyev, O.; Austin, A. J.; Cammi, R.; Pomelli, C.; Ochterski, J. W.; Martin, R. L.; Morokuma, K.; Zakrzewski, V. G.; Voth, G. A.; Salvador, P.; Dannenberg, J. J.; Dapprich, S.; Daniels, A. D.; Farkas, O.; Foresman, J. B.; Ortiz, J. V.; Cioslowski, J.; Fox, J. Rev. D. Gaussian o9, Gaussian, Inc., Wallingford CT. 2013.

(28) Alecu, I.; Zheng, J.; Zhao, Y.; Truhlar, D. G. J. Chem. Theory Comput. 2o10, 6, 2872-2887.

(29) Curtiss, L. A.; Raghavachari, K.; Redfern, P. C.; Baboul, A. G.; Pople, J. A. Chem. Phys. Lett. 1999, 314, 101-107.

(30) (a) Izgorodina, E. I.; Coote, M. L. J. Phys. Chem. A 2006, 110, 2486-2492; (b) Izgorodina, E. I.; Brittain, D. R.; Hodgson, J. L.; Krenske, E. H.; Lin, C. Y.; Namazian, M.; Coote, M. L. J. Phys. Chem. A 2007, 111, 10754-10768.

(31) Marenich, A. V.; Cramer, C. J.; Truhlar, D. G. J. Phys. Chem. B 2009, 113, 6378-6396.

(32) (a) Klamt, A. J. Phys, Chem. 1995, 99, 2224-2235; (b) Klamt, A.; Jonas, V.; Bürger, T.; Lohrenz, J. C. J. Phys. Chem. A 1998, 102,
5074-5085; (c) Klamt, A., COSMO-RS: from quantum chemistry to fluid phase thermodynamics and drug design. Elsevier: 2005.

(33) Louwen, J. N.; Pye, C. C.; Van Lenthe, E.; McGarrity, E. S.; Xiong, R.; Sandler, S. I.; Burnett, R. I., In http://www.scm.com, ADF2014 COSMO-RS, SCM, Theoreoretical Chemistry, Vrije Universiteit, Amsterdam, The Netherlands: 2014.

(34) Pye, C. C.; Ziegler, T.; Van Lenthe, E.; Louwen, J. N. Can. J. Chem. 2009, 87, 790-797.

(35) (a) Werner, H. J.; Knowles, P. J.; Knizia, G.; Manby, F. R.; Schütz, M. WIREs Comput. Mol. Sci. 2012, 2, 242-253; (b) Werner, H. J.; Knowles, P. J.; Knizia, G.; Manby, F. R.; Schütz, M.; Celani, P.; Györffy, W.; Kats, D.; Korona, T.; Lindh, R.; Mitrushenkov, A.; Rauhut, G.; Shamasundar, K. R.; Adler, T. B.; Amos, R. D.; Bernhardsson, A.; Berning, A.; Cooper, D. L.; Deegan, M. J. O.; Dobbyn, A. J.; Eckert, F.; Goll, E.; Hampel, C.; Hesselmann, A.; Hetzer, G.; Hrenar, T.; Jansen, G.; Köppl, C.; Liu, Y.; Lloyd, A. W.; Mata, R. A.; May, A. J.; McNicholas, S. J.; Meyer, W.; Mura, M. E.; Nicklass, A.; O'Neill, D. P.; Palmieri, P.; Peng, D.; Pflüger, K.; Pitzer, R.; Reiher, M.; Shiozaki, T.; Stoll, H.; Stone, A. J.; Tarroni, R.; Thorsteinsson, T.; Wang, M. MOLPRO, version 2015.1, a package of ab initio programs, http://www.molpro.net 2015. 


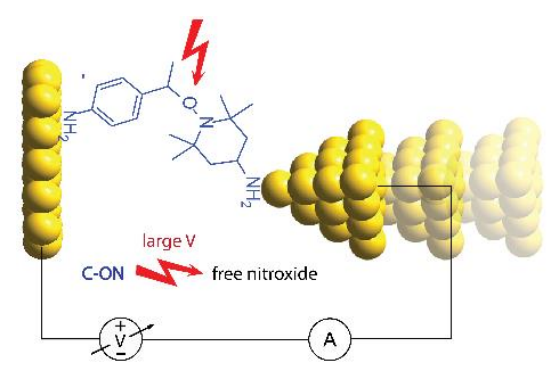




\section{Electrochemical and Electrostatic Cleavage of Alkoxyamines}

Long Zhang, ${ }^{\mathrm{a}, \mathrm{b}}$ Eduardo Laborda, ${ }^{\mathrm{c}}$ Nadim Darwish, ${ }^{*, \mathrm{a}}$ Benjamin B. Noble, ${ }^{\mathrm{d}}$ Jason H. Tyrell, ${ }^{\mathrm{d}}$ Sandra Pluczyk, ${ }^{e}$ Anton P. Le Brun, ${ }^{\dagger}$ Gordon G. Wallace, ${ }^{b}$ Joaquin Gonzalez, ${ }^{*, c}$ Michelle L. Coote, ${ }^{*, d}$ and Simone Ciampi ${ }^{*, a}$

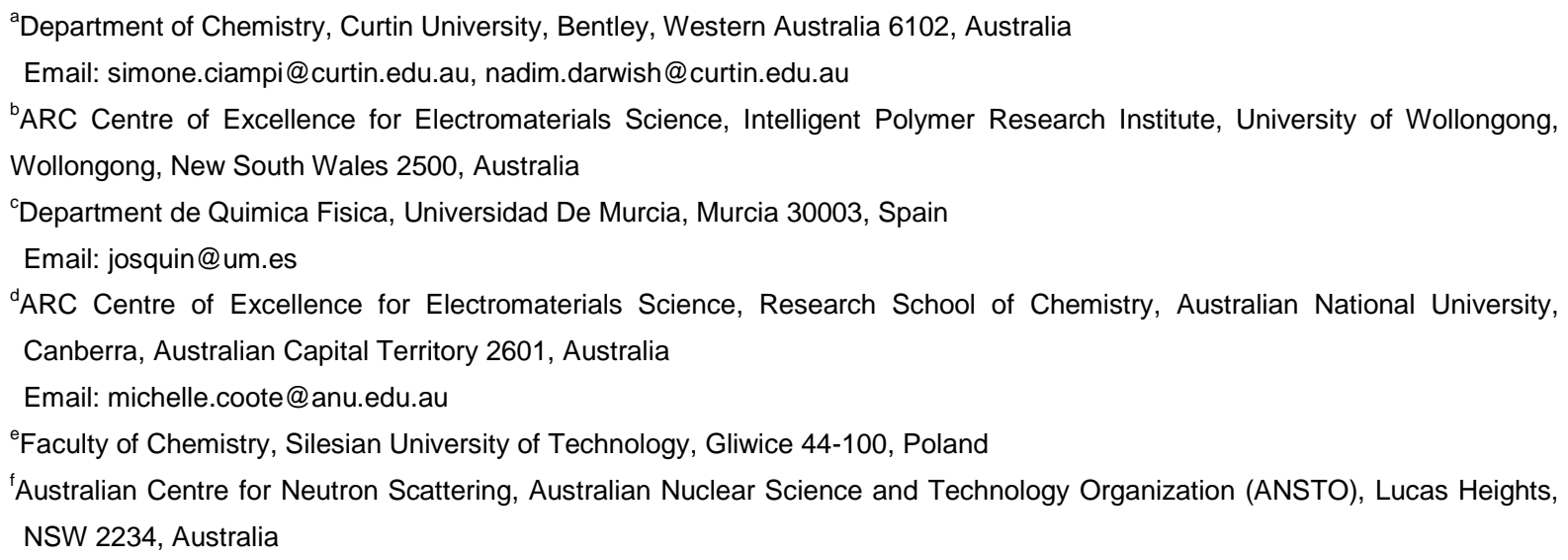

S1. Materials

S2. Purification and analysis of synthesized compounds S2

S3. STM-BJ experiments S5

S4. $\mathrm{Si}(100)$ surface modification S6

S5. Surface characterization S7

S6. Additional electrochemical data S9

S7. Theoretical study of the effect of external electric field on alkoxyamine cleavage .

S8. Theoretical study of the electrochemical cleavage of the alkoxyamine ... S35

S9. References $\mathrm{S} 50$ 


\section{S1. Materials}

Chemicals. Unless noted otherwise, all chemicals were of analytical grade and used as received. Milli- $\mathrm{Q}^{\mathrm{TM}}$ water $(>18$ $\mathrm{M} \Omega \mathrm{cm}$ ) was used to prepare solutions, for chemical reactions and in surface cleaning procedures. Acetonitrile (MeCN), dichloromethane (DCM), ethanol, and 2-propanol for substrate cleaning and chemical reactions were redistilled prior to use. Hydrogen peroxide (30 wt.\% in water), ammonium fluoride (PuranalTM, 40 wt.\% in water), and sulfuric acid (PuranalTM, 95-97\%) used in wafer cleaning and etching procedures were of semiconductor grade. 1,8-Nonadiyne (Sigma-Aldrich, 98\%) was redistilled from sodium borohydride (Sigma-Aldrich, $99+\%)$ under reduced pressure $\left(80^{\circ} \mathrm{C}\right.$, 10-12 Torr) and stored under a high purity argon atmosphere prior to use. Styrene (ReagentPlus $®$, Sigma-Aldrich $\geq 99 \%$ ) was washed with aqueous $5 \%$ sodium hydroxide for removing the inhibitors and/or stabilizers. Tetrabutylammonium $\left(\mathrm{Bu}_{4} \mathrm{~N}\right)$ salts (Sigma-Aldrich) of perchlorate $\left(\mathrm{ClO}_{4}, \geq 98 \%\right)$ and hexafluorophosphate $\left(\mathrm{PF}_{6}\right.$, purum, $\left.\geq 98 \%\right)$ were recrystallized two times from 2-propanol before using as supporting electrolytes. Sodium tetrakis[3,5-bis(trifluoromethyl)phenyl]borate (Alfa Aesar, NaBARF, 97\%, may cont. 1-5\% water) was directly used for a control supporting electrolyte without any refinements. 4-Vinylbenzyl chloride (90\%), 4-vinylaniline, di-tert-butyl dicarbonate $\left(\mathrm{BOC}_{2} \mathrm{O}, \quad\right.$ ReagentPlus $\left.{ }^{\circ}, \quad \geq 99 \%\right)$, ferrocene (Fc, 98\%), TEMPO (98\%) and 4-amino-2,2,6,6-tetramethylpiperidine-1-oxyl (4- $\mathrm{NH}_{2}$-TEMPO) were purchased from Sigma-Aldrich. 4-Azido-2,2,6,6-tetramethyl-1-piperdine-1-oxyl 1-(1-(4-aminophenyl)ethoxy)-2,2,6,6-tetramethylpiperidin-4-amine 4-azido-2,2,6,6-tetramethyl-1-(1-phenylethoxy)piperdine $\left(4-\mathrm{N}_{3}-\mathrm{TEMPO}\right)^{1}$, published methods with minor modifications ${ }^{2}$ (see the Section $\mathrm{S} 2$ below).

Silicon wafers. Prime grade, single-side polished silicon wafers, 100 -oriented $\left(<100> \pm 0.5^{\circ}\right)$, p-type (boron-doped), 500 $\pm 25 \mu \mathrm{m}$ thick, with nominal resistivity of $0.001-0.003 \Omega \mathrm{cm}$ were obtained from Siltronix, S.A.S. (Archamps, France).

\section{S2. Purification and analysis of synthesized compounds}

Thin-layer chromatography (TLC) was performed on silica gel using Merck aluminum sheets (60 F254). Merck $60 \AA$ silica gel (220-400 mesh particle size) was used for column chromatography. Heated electrospray ionisation - high resolution mass spectrometer (HESI-HRMS, Thermo Fisher Scientific Corporation, Waltham, USA) was used for high accuracy mass determination of the alkoxyamine compounds. All stock solutions were prepared by dissolving alkoxyamines into the minimal amount of either methanol or DCM. Solution were then diluted down to $10 \mu \mathrm{g} / \mathrm{mL}$ in $\mathrm{MeOH}$ containing $0.1 \%$ formic acid and infused these samples into the mass spectrometer at 5-25 $\mu \mathrm{L} / \mathrm{min}$ using a built-in syringe pump. Nuclear magnetic resonance (NMR) spectra were recorded on Brunker Avance 400 spectrometer. Alkoxyamine 2 (surface systems, silicon electrodes) was synthesized in one-step from4- $\mathrm{N}_{3}$-TEMPO, alkoxyamine 1 a (precursor of alkoxyamine 1 used in STM-BJ experiments, gold electrodes) was synthesized in one-step from nitroxide $\mathbf{A}$ and olefin $\mathbf{B}$. Synthetic details for $\mathbf{A}$ and $\mathbf{B}$ are shown below.

4-Tert-butoxycarbonylamino-2,2,6,6-tetramethylpiperidin-1-oxyl (A). Three molar equivalents of $\mathrm{NaHCO}_{3}(1.26 \mathrm{~g}, 15$

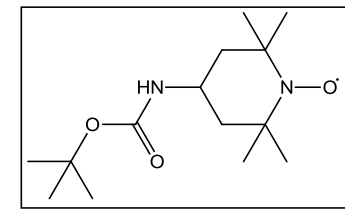
$\mathrm{mmol}$ ) and a $20 \%$ molar excess of $\mathrm{BOC}_{2} \mathrm{O}(1.31 \mathrm{~g}, 6 \mathrm{mmol})$ were added in portions to an ice-cold solution of 4-amino-TEMPO $(0.86 \mathrm{~g}, 5 \mathrm{mmol})$ in a mixture of THF/water $(1 / 1,50$ $\mathrm{mL})$. The reaction mixture was stirred under argon atmosphere in an ice-bath for $45 \mathrm{~min}$ followed by stirring overnight ( $12 \mathrm{~h})$ at room temperature. The crude solution was poured into $100 \mathrm{~mL}$ ether and the organic phases were washed with water $(3 \times 50 \mathrm{~mL})$. The combined organic layers were then dried over $\mathrm{MgSO}_{4}$, and the ether was removed under reduced pressure. The obtained orange solid residue was purified by silica gel column chromatography (ethyl acetate/hexane, $1 / 4, v / v)$ to give an orange solid that was used in the following step without further characterization (1.14 g, $4.2 \mathrm{mmol})$.

Tert-butyl-4-vinylphenylcarbamate (B). To a stirred solution of 4-vinylaniline $(1.79 \mathrm{~g}, 15 \mathrm{mmol})$ in $30 \mathrm{~mL}$ anhydrous

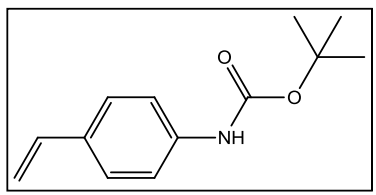
$\mathrm{THF}$, a solution of $\mathrm{BOC}_{2} \mathrm{O}(3.93 \mathrm{~g}, 18 \mathrm{mmol})$ in anhydrous THF $(30 \mathrm{~mL})$ was added dropwise at room temperature under nitrogen atmosphere while stirring. The solution was refluxed overnight ( $12 \mathrm{~h}$ ), the mixture was then allowed to cool to room temperature, diluted with DCM and then partitioned between water and DCM. The aqueous solution was further extracted with DCM $(3 \times 50 \mathrm{~mL})$, the organic layers were combined, washed 
with water and brine, then dried over $\mathrm{MgSO}_{4}$. After removal of the solid residue by filtration, the solvent was removed under reduced pressure and the crude material was purified by silica gel column chromatography (ethyl acetate/hexane, $1 / 10, \mathrm{v} / \mathrm{v})$ to yield the title compound as a white solid $(2.8 \mathrm{~g}, 12.8 \mathrm{mmol})$.

${ }^{1} \mathrm{H}$ NMR $(400 \mathrm{MHz}, d$-DMSO): $\delta(\mathrm{ppm})=9.38$ (s, BOC-NH, 1H), $7.43(\mathrm{~d}, \mathrm{Ar}-\mathrm{H}, J=8.68 \mathrm{~Hz}, 2 \mathrm{H}), 7.35$ (d, Ar-H, J=8.56 $\mathrm{Hz}, 2 \mathrm{H}$ ), 6.63 (dd, $\left.\mathrm{Ar}-\mathrm{CH}=\mathrm{CH}_{2}, J=17.62 \mathrm{~Hz}, 10.92 \mathrm{~Hz}, 1 \mathrm{H}\right), 5.68$ (dd, $\mathrm{Ar}-\mathrm{CH}=\mathrm{CH}_{2}, J=17.70 \mathrm{~Hz}, 1.06 \mathrm{~Hz}, 1 \mathrm{H}$ ), 5.12 (dd, $\left.\mathrm{Ar}-\mathrm{CH}=\mathrm{CH}_{2}, J=10.92 \mathrm{~Hz}, 1.00 \mathrm{~Hz}, 1 \mathrm{H}\right), 1.47$ (s, BOC, 9H).

${ }^{13} \mathrm{C}$ NMR (400 MHz, d-DMSO): $\delta(p p m)=152.64,139.26,136.20,131.00,126.48,117.96,112.08,79.06,28.09$.

\section{Alkoxyamines}

1-(1-(4-tert-butylcarbamatephenyl)ethoxy)-2,2,6,6-tetramethylpiperidin-4-tert-butoxycarbonylamino

syntheses.

(di-BOC-amino-TEMPO-PE, 1a), 4-N $\mathrm{N}_{3}$-TEMPO-PE (2) and TEMPO-PE (3) were synthesized via the following procedures. To a $100 \mathrm{~mL}$ rounded-bottom flask equipped with a magnetic stirrer and placed in an ice-bath, we added sequentially i) $2 \mathrm{mmol}$ of the nitroxide precursors dissolved in $60 \mathrm{~mL}$ of a toluene/ethanol $(1 / 1, \mathrm{v} / \mathrm{v}) \mathrm{mixture}$, ii) a ten-fold molar excess of styrene or its derivatives and iii) $\mathrm{Mn}(\mathrm{OAc})_{3} 2 \mathrm{H}_{2} \mathrm{O}(5.36 \mathrm{~g}, 20 \mathrm{mmol})$. A 15-fold molar excess of $\mathrm{NaBH}_{4}$ (with respect to the nitroxide) was added in portions at 15-min intervals. After stirring overnight under nitrogen atmosphere, the crude reaction mixture was filtered, the filtrate was transferred into water and the aqueous suspension was then washed three times with DCM. The combined organic layers were evaporated under reduced pressure and the crude materials were purified on silica gel column chromatography using different eluents to yield either 1a (ethyl acetate/hexane, 1/5, v/v, white solid, $0.42 \mathrm{~g}, 0.9 \mathrm{mmol}$ ), 2 (ethyl acetate/hexane, 1/25, v/v, pale yellow oil liquid, $0.33 \mathrm{~g}$, $1.1 \mathrm{mmol}$ ), or 3 (hexane, white waxy solid, $0.32 \mathrm{~g}, 1.2 \mathrm{mmol}$ ). Di-amino-TEMPO-PE (1) di-BOC-amino-TEMPO-PE (1a, 1 $\mathrm{g}, 2 \mathrm{mmol}$ ) was deprotected with $\mathrm{CF}_{3} \mathrm{COOH}(6.84 \mathrm{~g}, 60 \mathrm{mmol})$ in $20 \mathrm{~mL} \mathrm{DCM}$ with stirring at room temperature. After $6 \mathrm{~h}$ of stirring, the reaction mixture was adjusted to neutral $\mathrm{pH}$ with saturated $\mathrm{NaHCO}_{3}$ aqueous solution and the water phase was then extracted with DCM $(3 \times 20 \mathrm{~mL})$. The combined organic extracts were dried over $\mathrm{MgSO}_{4}$, filtered, and then concentrated under reduced pressure. Alkoxyamine 1 was purified by chromatography over an alkalified silica gel column (ethyl acetate $/ \mathrm{MeOH}, 5 / 1 \mathrm{v} / \mathrm{v}$ with $1 \% \mathrm{v} / \mathrm{v}$ of $\mathrm{Et}_{3} \mathrm{~N}$ ) as a yellow oil $(0.18 \mathrm{~g}, 0.6 \mathrm{mmol})$.

${ }^{1} \mathrm{H}$ NMR (1a, $400 \mathrm{MHz}, d$-DMSO) $\delta(\mathrm{ppm})=9.27$ (s, BOC-NH-Ar, 1H), $7.38(\mathrm{~d}, \mathrm{Ar}-\mathrm{H}, J=8.28 \mathrm{~Hz}, 2 \mathrm{H}), 7.16(\mathrm{~d}, \mathrm{Ar}-\mathrm{H}, J=$ $8.52 \mathrm{~Hz}, 2 \mathrm{H}), 6.64$ (d, BOC-NH-TEMPO, $J=7.88 \mathrm{~Hz}, 1 \mathrm{H}), 4.65$ (q, NO-CH-Ar, $J=6.56 \mathrm{~Hz}, 1 \mathrm{H}), 3.62-3.48(\mathrm{~m}, 1 \mathrm{H})$, 1.50-1.34 (m, 24H), 1.28-1.19 (m, 4H), $1.14(\mathrm{~s}, 3 \mathrm{H}), 0.99(\mathrm{~s}, 3 \mathrm{H}), 0.55(\mathrm{~s}, 3 \mathrm{H})$.

${ }^{13} \mathrm{C}$ NMR (1a, $400 \mathrm{MHz}, d$-DMSO): $\delta(\mathrm{ppm})=154.84,152.76,138.54,138.36,126.87,117.76,82.10,78.88,77.49,59.62$, $59.17,45.67,45.13,33.78,28.25,28.12,22.80,20.61$

HRMS (1a, $\mathrm{m} / \mathrm{z}):[\mathrm{M}+\mathrm{H}]^{+}$calcd for $\mathrm{C}_{27} \mathrm{H}_{46} \mathrm{~N}_{3} \mathrm{O}_{5}$ 492.3432, found 492.3428 .

${ }^{1} \mathrm{H}$ NMR $\left(1,400 \mathrm{MHz}, d\right.$-DMSO) $\delta(\mathrm{ppm})=7.32(\mathrm{~d}, \mathrm{Ar}-\mathrm{H}, J=8.48 \mathrm{~Hz}, 2 \mathrm{H}), 7.23(\mathrm{~d}, \mathrm{Ar}-\mathrm{H}, J=8.54 \mathrm{~Hz}, 2 \mathrm{H}), 6.35\left(\mathrm{~s}, \mathrm{NH}_{2}-\mathrm{Ar}\right.$, 2H), $5.56\left(\mathrm{~s}, \mathrm{NH}_{2}\right.$-TEMPO, 2H), $4.76(\mathrm{q}, \mathrm{NO}-\mathrm{CH}-\mathrm{Ar}, \mathrm{J}=6.52 \mathrm{~Hz}, 1 \mathrm{H}), 2.97-2.85(\mathrm{~m}, 1 \mathrm{H}), 1.72-1.62(\mathrm{~m}, 1 \mathrm{H}), 1.57-1.48(\mathrm{~m}$, $1 \mathrm{H}), 1.46-1.40(\mathrm{~m}, 3 \mathrm{H}), 1.31-1.23(\mathrm{~m}, 4 \mathrm{H}), 1.20-1.16(\mathrm{~m}, 4 \mathrm{H}), 1.02(\mathrm{~s}, 3 \mathrm{H}), 0.61(\mathrm{~s}, 3 \mathrm{H})$.

HRMS $(1, m / z):[M+H]^{+}$calcd for $\mathrm{C}_{17} \mathrm{H}_{30} \mathrm{~N}_{3} \mathrm{O} 292.2383$, found 292.2381 .

${ }^{1} \mathrm{H}$ NMR (2, $400 \mathrm{MHz}, d$-DMSO) $\delta=7.36-7.27$ (m, Ar-H, 4H), 7.26-7.20 (m, Ar-H, 1H), 4.75 (q, NO-CH-Ar, J = 6.76 $\mathrm{Hz}, 1 \mathrm{H}), 3.80\left(\mathrm{~m}, \mathrm{~N}_{3}-\mathrm{CH}, 1 \mathrm{H}\right), 1.87-1.78(\mathrm{~m}, 1 \mathrm{H}), 1.73-1.64(\mathrm{~m}, 1 \mathrm{H}), 1.48-1.37(\mathrm{~m}, 4 \mathrm{H}), 1.35-1.25(\mathrm{~m}, 4 \mathrm{H}), 1.18(\mathrm{~s}, 3 \mathrm{H})$, $1.03(\mathrm{~s}, 3 \mathrm{H}), 0.62(\mathrm{~s}, 3 \mathrm{H})$.

${ }^{13} \mathrm{C}$ NMR $(2,400 \mathrm{MHz}, d$-DMSO): $\delta(\mathrm{ppm})=144.78,128.07,127.05,126.44,82.60,59.50,59.21,44.28,44.17,33.75$, 33.52, 23.01, 20.45

$\operatorname{HRMS}(2, \mathrm{~m} / \mathrm{z}):[\mathrm{M}+\mathrm{H}]^{+}$calcd for $\mathrm{C}_{17} \mathrm{H}_{27} \mathrm{~N}_{4} \mathrm{O} 303.2185$; found 303.2193.

${ }^{1} \mathrm{H}$ NMR $(3,400 \mathrm{MHz}, d$-DMSO) $\delta(\mathrm{ppm})=7.34-7.28(\mathrm{~m}, \mathrm{Ar}-\mathrm{H}, 4 \mathrm{H}), 7.25-7.20(\mathrm{~m}, \mathrm{Ar}-\mathrm{H}, 1 \mathrm{H}), 4.75$ (q, NO-CH-Ar, $J=6.52$ $\mathrm{Hz}, 1 \mathrm{H}), 1.54-1.38(\mathrm{~m}, 6 \mathrm{H}), 1.37-1.19(\mathrm{~m}, 6 \mathrm{H}), 1.12(\mathrm{~s}, 3 \mathrm{H}), 0.97(\mathrm{~s}, 3 \mathrm{H}), 0.59(\mathrm{~s}, 3 \mathrm{H})$.

${ }^{13} \mathrm{C}$ NMR $(3,400 \mathrm{MHz}, d$-DMSO): $\delta(\mathrm{ppm})=145.14,128.02,126.90,126.37,82.36,59.17,59.03,34.02,33.79,23.18$, $20.08,16.67$.

$\operatorname{HRMS}(3, \mathrm{~m} / \mathrm{z}):[\mathrm{M}+\mathrm{H}]^{+}$calcd for $\mathrm{C}_{17} \mathrm{H}_{28} \mathrm{NO} 262.2171$; found 262.2161. 

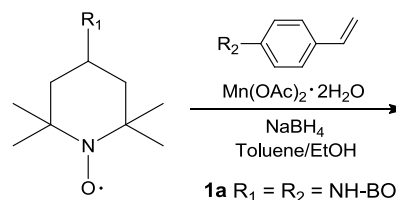<smiles>CC(ON1C(C)(C)CC(Br)CC1(C)C)c1ccc(Br)cc1</smiles>
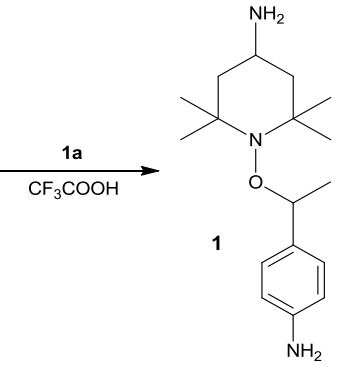

Scheme S1. Syntheses of the alkoxyamines used in this study; 1 (STM-BJ experiments on gold surfaces and theoretical models), 2 (electrochemistry on silicon surface systems), 3 (electrochemistry diffusive systems, EPR and theoretical modelling). 


\section{S3. STM-BJ experiments}

Conductance measurements via scanning-tunneling microscopy single-molecule break junctions experiments (STM-BJ) were used to track the chemical fate of an alkoxyamine molecule (di-amino-TEMPO-PE, 1) under electric fields of variable magnitude. Both the parent molecule (i.e. 1) as well as its putative fragments upon homolysis were detected as discrete electrical signatures by the STM-BJ technique. These experiments acted as a direct test of the link between electrostatic field and the bond-breaking event in $\mathbf{1}$ (C-O lysis) when this molecule is bridged between gold electrodes.

a)
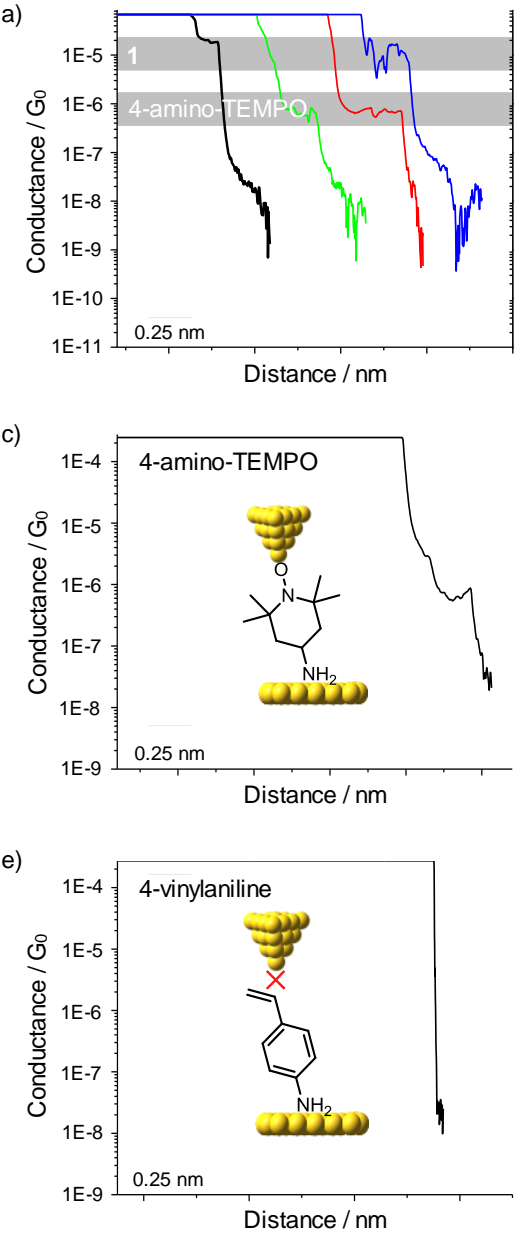

b)

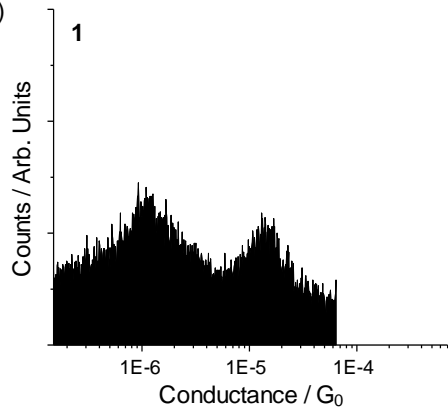

d)
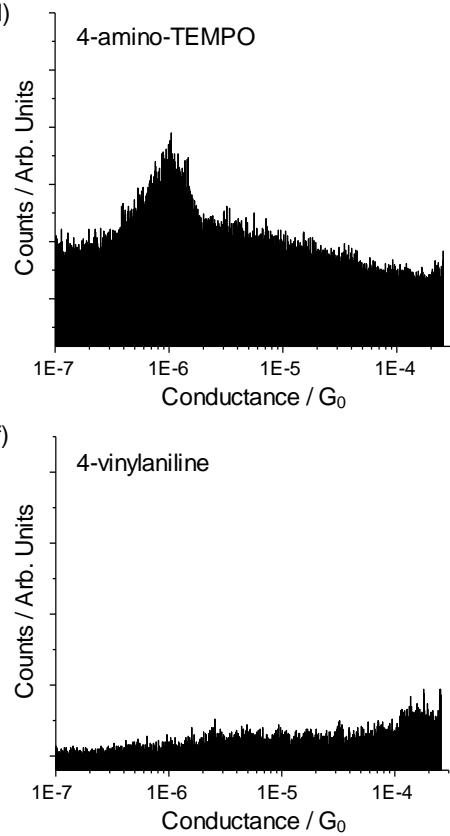

Figure S1. Electrostatic catalysis in the homolysis of alkoxyamines. (a,b) Representative single-molecule STM-BJ conductance "pull" trace and statistical conductance histograms for samples of alkoxyamine 1 in mesitylene/DCM biased at $200 \mathrm{mV}$ (gold STM tip-to-Au(111) substrate bias). The histograms that are built from the analysis of ca. 4000 pulling traces show the coexistence of the $1 \mathrm{E}-5$ Go "signature" which is assigned to the parent molecule (1) with the $1 \mathrm{E}-6 \mathrm{G}_{0}$ population which is assigned to one of the lysis product (4-amino-TEMPO molecules, i.e. the putative nitroxide fragment originated from the $\mathrm{C}-\mathrm{O}$ lysis in 1). (c,d) STM-BJ control experiments of the molecule 4-amino-TEMPO held at $50 \mathrm{mV}$ showing a representative conductance-distance trace alongside conductance histograms revealing a major contributor of electrical conductance centred at $1 \mathrm{E}-6 \mathrm{G}_{0}$. The conductance does not change between $50 \mathrm{mV}$ and $300 \mathrm{mV}$ (Figure 1 in main text) for the 4-amino-TEMPO control, indicating that this molecule not affected by the electric field in this bias range and remains intact during the measurement. (e,f) STM-BJ experiments on samples of 4-vinylaniline molecule, one of the putative product of the lysis of 1. Plateaus are absent from the STM-BJ curves and this is statistically evident as no "peak" can be observed in conductance histograms built from the data. It can be inferred that the original alkoxyamine molecule $\mathbf{1}$ breaks in response to an electric field (threshold of about $200 \mathrm{mV}$ bias between the gold STM tip and the $\mathrm{Au}(111)$ substrate) to release a 4-amino-TEMPO fragment (electrical "fingerprinting" of $1 \mathrm{E}^{-6} \mathrm{G}_{0}$ ) and possibly a vinyl aniline that however is not forming a top \& bottom junction with the gold electrodes. 


\section{S4. $\mathrm{Si}(100)$ surface modification}

Assembly of monolayers of 1,8-nonadiyne on Si(100). Silicon (100) wafers were firstly cut into pieces (approximately $10 \times 30 \mathrm{~mm}$ in size), cleaned by $30 \mathrm{~min}$ of Soxhlet reflux with chloroform and immersed for 20-30 min in hot Piranha solution $\left(100{ }^{\circ} \mathrm{C}\right.$, a $3: 1(\mathrm{v} / \mathrm{v})$ mixture of concentrated sulfuric acid to $30 \%$ hydrogen peroxide Caution: piranha solution reacts violently with organic substances). The wafers were then rinsed with copious water and etched with a deoxygenated $40 \%$ aqueous ammonium fluoride solution for $5 \mathrm{~min}$. The assembly of the acetylenylated $\mathrm{Si}(100)$ surface by covalent attachment of 1,8-nonadiyne on hydrogen-terminated silicon followed a previously reported procedure (Scheme S2). ${ }^{3}$ The freshly etched hydrogen-terminated surface samples were then transferred to a degassed (through a minimum of $30 \mathrm{~min}$ of argon bubbling) sample of the diyne held in a reaction vessel (a custom-made Schlenk flask). The samples were kept under a stream of argon while the reaction vessel was immersed in an oil bath set to $160^{\circ} \mathrm{C}$. After $3 \mathrm{~h}$ the flask was opened to the atmosphere, and the functionalized surface samples were rinsed several times with DCM and rested for a $12 \mathrm{~h}$ period in a sealed vial at $+4{ }^{\circ} \mathrm{C}$ under $\mathrm{DCM}$, before being further reacted with 2 (4- $\left.\mathrm{N}_{3}-\mathrm{TEMPO}-\mathrm{PE}\right)$ or $4-\mathrm{N}_{3}$-TEMPO.

Click derivatization of acetylene-terminated Si (100) surface. In the "click" procedure, copper(I)-catalyzed alkyne-azide cycloaddition (CUAAC) reactions were used to graft 4- $\mathrm{N}_{3}$-TEMPO-PE (2) to the acetylenyl surface. ${ }^{4}$ In brief, to a reaction vial containing the alkyne-functionalized silicon surface was added (i) the azide $\left(2,0.5 \times 10^{-3} \mathrm{M}\right.$, 2-propanol/water, 1:1), (ii) copper(II) sulfate pentahydrate $\left(1.0 \times 10^{-4} \mathrm{M}\right)$ and (iii) sodium ascorbate $(5 \mathrm{mg} / \mathrm{mL})$. Reactions were carried out at room temperature, without excluding air from the reaction environment and under ambient light. Samples were removed from the reaction vessel after a reaction time of $2 \mathrm{~h}$. The surface-bound [1,2,3]-triazoles $\mathrm{Si}(100)$ samples (S-1) were rinsed consecutively with copious amounts ethanol, water, ethanol and DCM and blown dry in argon before being analyzed. An analogous procedure was used for the attachment of 4- $\mathrm{N}_{3}$-TEMPO to yield redox-active monolayers of TEMPO (S-2, Scheme S2) used as control experiments.

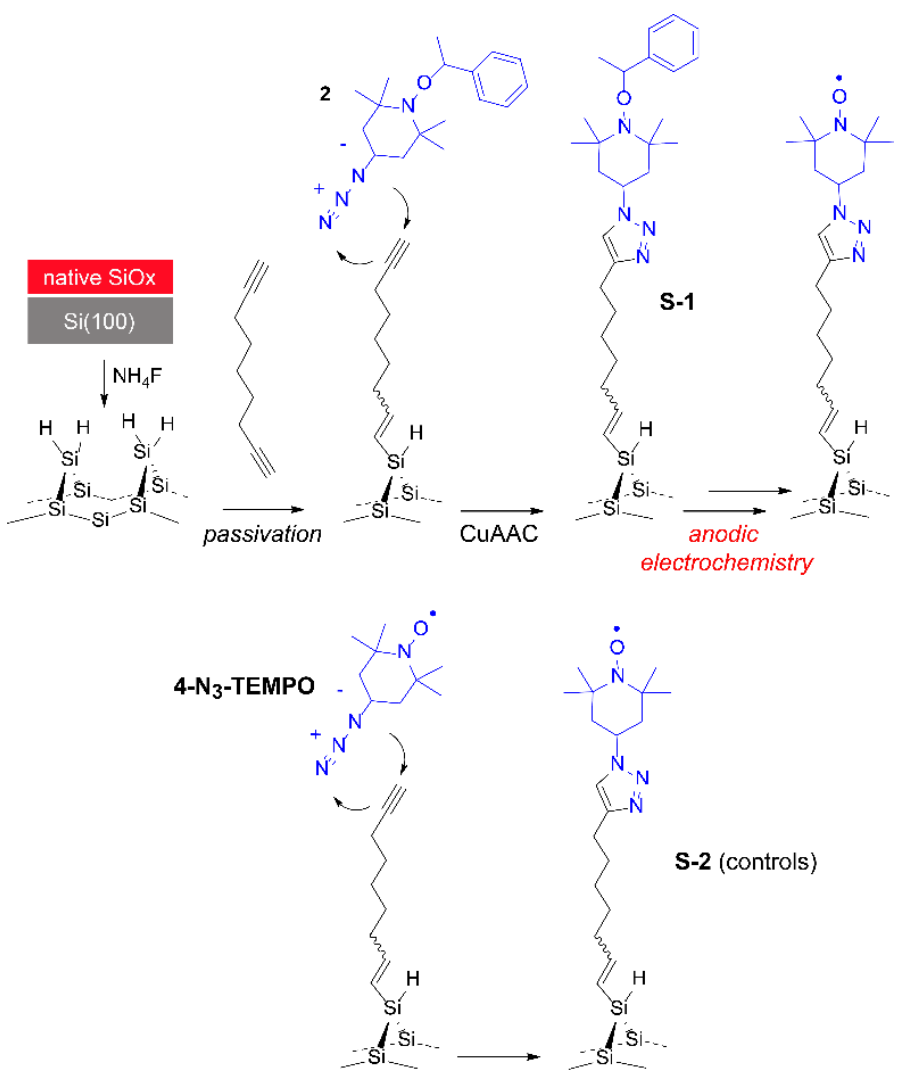

Scheme S2. Thermal hydrosilylation of 1,8-nonadiyne to passivate an hydrogen-terminated $\mathrm{Si}(100)$ electrode and covalent attachment of 2 (upper panel) and 4- $\mathrm{N}_{3}$-TEMPO (lower panel) via CuAAC "click" reactions to yield either alkoxyamine (S-1) or nitroxide (S-2, controls) monolayers, respectively. 


\section{S5. Surface characterization}

$X$-ray photoelectron spectroscopy (XPS). The formation of the alkoxyamine monolayer (S-1) was confirmed by XPS measurements. X-ray photoelectron spectra (XPS) were obtained on an ESCALAB 220iXL spectrometer fitted with a monochromatic Al Ka source (1486.6 eV), a hemispherical analyzer and a 6 multi-channel detector. Spectra were recorded in normal emission with the analyzing chamber operating below $10^{-9} \mathrm{mbar}$. The resolution of the spectrometer is ca. $0.6 \mathrm{eV}$ as measured from the $\mathrm{Ag} 3 \mathrm{~d}_{5 / 2}$ signal (full width at half maximum, FWHM) with $20 \mathrm{eV}$ pass energy. High-resolution scans were run with $0.1 \mathrm{eV}$ step size, dwell time of $100 \mathrm{~ms}$ and the analyzer pass energy set to $20 \mathrm{eV}$. After background subtraction, spectra were fitted with Voigt functions. From the high resolution scans in Figure S2 it is evident that alkoxyamine $\mathbf{2}$ is covalently attached to the electrode. The electrode oxidation is also negligible. High-resolution scan for the $\mathrm{Si} 2 \mathrm{p}$ region comprising two spin-orbit-split components are shown in panel (b) and the silica-related emission (102-104 eV signal associated with $\mathrm{SiO}_{\mathrm{x}}$ ) were below the spectrometer detection limit of ca. $<0.05$ $\mathrm{SiO}_{x}$ monolayers equivalents. The XPS $\mathrm{C}$ 1s region can be deconvoluted into three contributions of mean binding energies of 283.4, 284.8, 286.2 eV (panel (c)). The smallest peak centered at 283.4 eV is ascribed to sp2 C-C in benzene ring. ${ }^{5}$ The peak at $284.8 \mathrm{eV}$ is characteristic of carbon-bound carbon atoms (C-C). Signals from C-O and C-N are suggested to contribute to the $286.2 \mathrm{eV}$ signal. ${ }^{6}$ The high-resolution $\mathrm{N}$ 1s data (panel (d)) shows good agreement with the literature for analogous derivatization procedures on solid surfaces. ${ }^{3 a}$ The lack of ca. $405 \mathrm{eV}^{7} \mathrm{~N} 1 \mathrm{~s}$ emission from electron-deficient nitrogen atom in the azido group confirm negligible physisorption of alkoxyamine 2 molecules in the film. The $\mathrm{N}$ 1s peak is resolved into two peaks assigned at 400.2 and $401.6 \mathrm{eV}$, with an expected ratio of the peak areas being ca. 3:1, which indicates a successful triazole formation. ${ }^{3 a, 8}$ There are no any binding energy signals for the free nitroxide nitrogen atom ${ }^{9}$ or a shakeup satellite of $\mathrm{N}-\mathrm{O}$ electrons, ${ }^{10}$ which confirms that the chemical structure of alkoxyamine 2 is preserved upon surface attachment. ${ }^{11}$
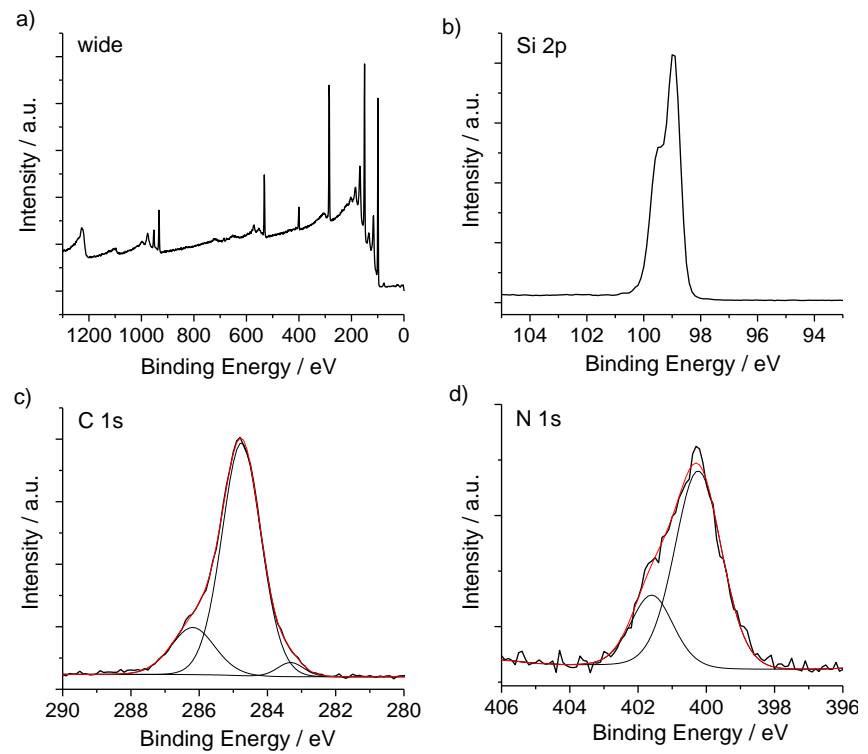

Figure S2. XPS spectra of 4- $\mathrm{N}_{3}$-TEMPO-PE monolayers (S-1) assembled on highly-doped $\mathrm{Si}(100)$ electrodes by CuAAC reactions between 4- $\mathrm{N}_{3}$-TEMPO-PE (2) and monolayers of 1,8-nonadiyne on Si(100). (a) Survey XPS scan. (b) High-resolution XPS scan for the Si $2 p$ region. (c) Narrow scan of the $\mathrm{C} 1 \mathrm{~s}$ region. (d) Narron scan of the $\mathrm{N} 1 \mathrm{~s}$ region.

$X$-ray reflectometry X-ray reflectivity (XRR). XRR profiles of S-1 samples (Figure S3) were measured under ambient conditions on a Panalytical Ltd X'Pert Pro Reflectometer using radiation wavelength of $\lambda=1.54 \AA$ from a Cu Ka source. The X-ray beam was focused and collimated using a Göbel mirror and pre-sample slit with a $0.1 \mathrm{~mm}$ horizontal opening. The reflectivity data was collected over an incident angle range of $0.05^{\circ}$ to $5.00^{\circ}$ in $0.01^{\circ}$ step sizes. A counting time of 7 seconds per step was used. All data was collected in the specular regime where angle of incidence is equal to angle of reflection. The data was normalized so that the critical edge is equal to unity and presented as reflectivity versus momentum transfer, $Q(=4 \pi \sin \theta / \lambda$, where $\theta$ is the angle of incidence and $\lambda$ the wavelength). Structural parameters were refined using MOTOFIT analysis software. ${ }^{11}$ The model used described the 4- $\mathrm{N}_{3}$-TEMPO-PE monolayer (S-1) was as single layer defined by its thickness, roughness and X-ray scattering length density (SLD) calculated as:

$\operatorname{SLD}=\left(\sum \mathrm{Z}_{\mathrm{i}} \mathrm{r}_{\mathrm{e}}\right) / \mathrm{V}_{\mathrm{m}}$

Where $Z_{i}$ is the atomic number for each atom in 4- $N_{3}$-TEMPO-PE, $r_{e}$ is the Bohr electron radius $\left(2.818 \times 10^{-15} \mathrm{~m}\right)$ and $V_{m}$ is the molecular volume (calculated to be $471.29 \AA^{3}$ from http://www.molinspiration.com/cgi-bin/properties). This gives a theoretical SLD for S-1 of $15.06 \times 10^{-6} \AA^{-2}$. In the fitting a least squares regression employing a genetic algorithm was 
used to minimize $x^{2}$ values by varying the thickness and SLD of the monolayer and the roughness of the monolayer-air and silicon-monolayer interfaces. The errors reported from the fitting are \pm 1 standard deviation.

The refined XRR parameters results of S-1 monolayers are listed in the table below:

\begin{tabular}{cccc}
\hline Layer & Thickness $(\AA)$ & SLD $\left(\times 10^{-6} \AA^{-2}\right)$ & Roughness $(\AA)$ \\
\hline Air & - & 0.0 (fixed parameter) & - \\
S-1 & $14.2 \pm 0.4$ & $15.0 \pm 0.1$ & $8.9 \pm 0.1$ \\
Silicon & - & 20.1 (fixed parameter) & $2.7 \pm 0.2$ \\
\hline
\end{tabular}

The fitted SLD gives a volume fraction of 0.99 for the S-1 monolayer. Therefore, a near-complete monolayer is formed.

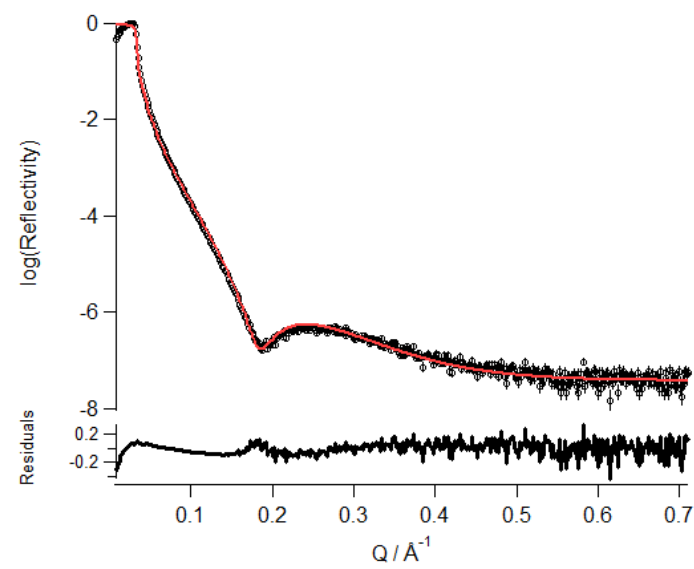

Figure S3. X-ray reflectivity of a 4- $\mathrm{N}_{3}$-TEMPO-PE monolayer (S-1) on silicon at the air-solid interface. The points with error bars show the collected data and the solid red line is the fit to the data. The lower trace is the residuals between collected and fitted data. The refined monolayer thickness is $14.2 \pm 0.4 \AA$ which is in good agreement with the theoretical values of $17 \AA$ (Chem3D). 
S6. Additional electrochemical data
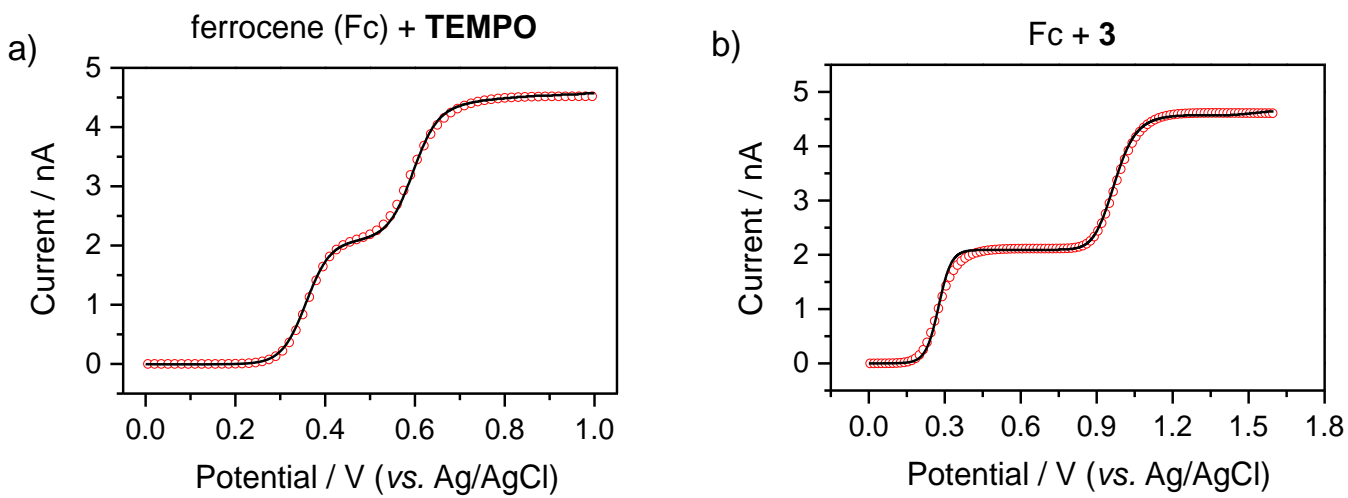

Figure S4. a) Cyclic voltammograms at Pt microdisks ( $4.5 \mu \mathrm{m}$ in radius) of a solution containing $0.53 \times 10^{-3} \mathrm{M} \mathrm{FC}$ and $0.63 \times 10^{-3} \mathrm{M} \mathrm{TEMPO}$ in $\mathrm{MeCN}$ with $1.0 \times 10^{-1} \mathrm{M} \mathrm{Bu}_{4} \mathrm{NClO}_{4}$ (voltage sweep rate is $\left.10 \mathrm{mV} \mathrm{s}^{-1}\right)$. The refined parameters $(\mathrm{E} \mathrm{mechanism})$ are: $E^{0}\left(\mathrm{Fc} / \mathrm{Fc}^{+}\right)=0.35 \mathrm{~V}$ vs. $\mathrm{Ag} / \mathrm{AgCl}$ ("leakless", see experimental section), $k^{0}\left(\mathrm{Fc} / \mathrm{Fc}^{+}\right)=0.20 \mathrm{~cm} \mathrm{~s}^{-1}, E^{0}$ (TEMPO/oxoammonium) $=0.58 \mathrm{~V}, k^{0}($ TEMPO/oxoammonium) $=$ $0.10 \mathrm{~cm} \mathrm{~s}^{-1}, \alpha=0.5$. b) Cyclic voltammograms at Pt microdisks $\left(4.5 \mu \mathrm{m}\right.$ in radius) of $0.53 \times 10^{-3} \mathrm{M} \mathrm{FC}$ and $0.92 \times 10^{-3} \mathrm{M} 3$ in MeCN with $1.0 \times$ $10^{-1} \mathrm{M} \mathrm{Bu}_{4} \mathrm{NClO}_{4}$ (scan rate is $\left.10 \mathrm{mV} \mathrm{s}^{-1}\right)$. The refined parameters $\left(\mathrm{EC}_{\text {irr }} \mathrm{E}\right.$ mechanism) are: $E^{0}\left(\mathrm{Fc} / \mathrm{Fc}^{+}\right)=0.26 \mathrm{~V} \mathrm{vs} \mathrm{Ag/AgCl}\left(\right.$ "leakless"), $k^{0}$ $\left(\mathrm{Fc} / \mathrm{Fc}^{+}\right)=0.10 \mathrm{~cm} \mathrm{~s}^{-1}, E^{0}\left(3 / 3^{\circ+}\right)=1.05 \mathrm{~V}, k^{0}\left(3 / 3^{\cdot+}\right)=0.08 \mathrm{~cm} \mathrm{~s}^{-1}, E^{0}($ TEMPO/oxoammonium $)=0.47 \mathrm{~V}, k^{0}($ TEMPO/oxoammonium) $=0.08 \mathrm{~cm}$ $\mathrm{s}^{-1}, \alpha=0.5$. For the chemical step of $3^{*+} \rightarrow$ TEMPO $+\mathrm{R}^{+}$, the refined $k_{\mathrm{f}}$ is $5.0 \times 10^{6} \mathrm{~s}^{-1}$. The experimental and simulated curves are plotted as solid lines and empty symbols, respectively. The diffusivity of ferrocene (Fc in shorthand) was set equal to $2.3 \times 10^{-5} \mathrm{~cm}^{2} \mathrm{~s}^{-1}$. ${ }^{12}$ The diffusivity of the $3 / 3^{++}$couple was $7.8 \times 10^{-6} \mathrm{~cm}^{2} \mathrm{~s}^{-1}$. The refined $D$ value of $2.2 \times 10^{-5} \mathrm{~cm}^{2} \mathrm{~s}^{-1}$ for the TEMPO/oxoammonium couple was also independently estimated by fittings of experimental cyclic voltammetry at Pt macrodisk electrodes (Figure S8). 

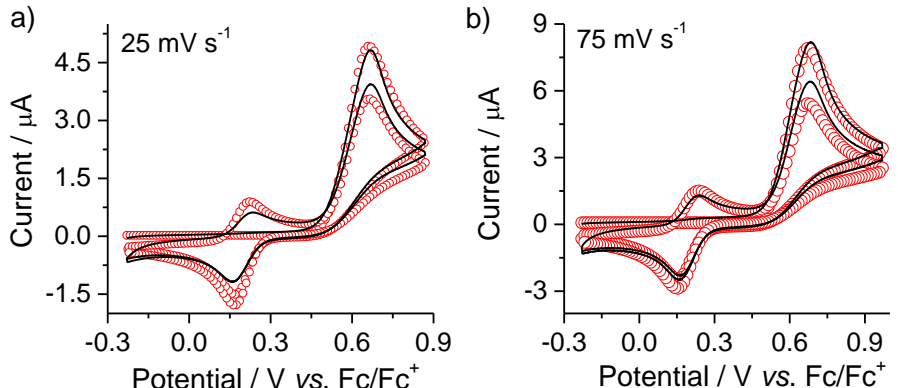

d)

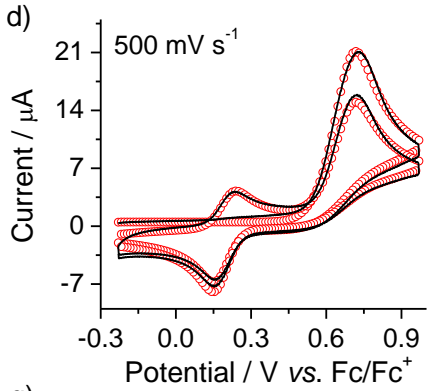

g)

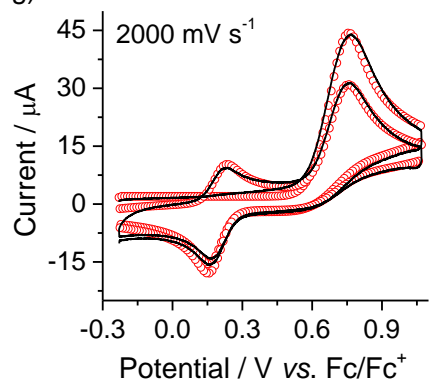

e)

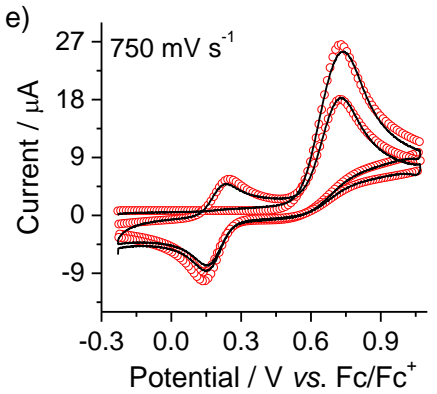

h)

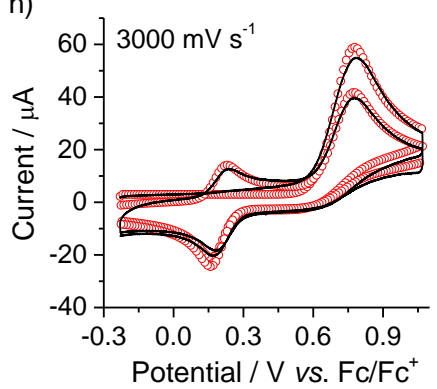

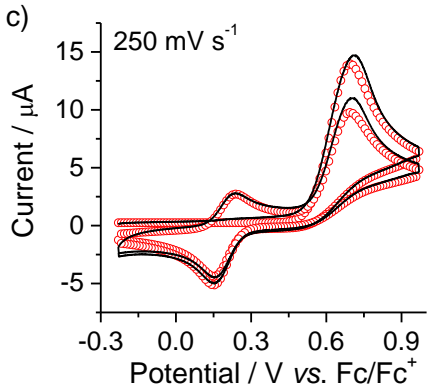

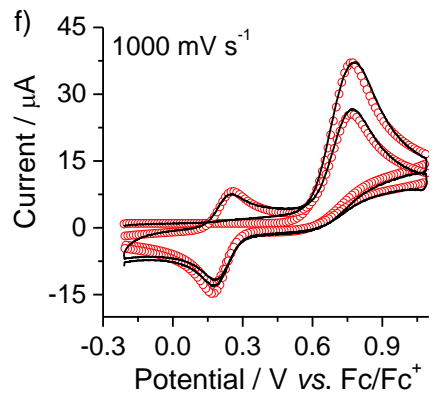

i)

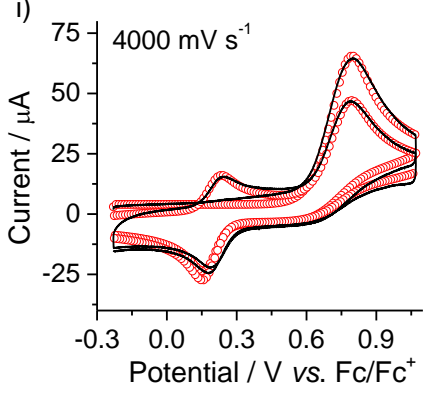

Figure S5. Anodic electrochemistry of alkoxyamine 3. Experimental (solid line) and simulated (empty symbols) voltammograms at different scan rates (25 to $4000 \mathrm{mV} \mathrm{s}^{-1}$, as specified by labels in figure) of $0.5 \times 10^{-3} \mathrm{M} 3$ in MeCN with $1.0 \times 10^{-1} \mathrm{M} \mathrm{Bu}_{4} \mathrm{NClO}_{4}$. Best-fit parameters (EC irr $\mathrm{E}$ mechanism) are: $E_{1}^{0}=0.78 \mathrm{~V}$ (vs. Fc/Fc $\left.{ }^{+}\right), k_{1}^{0}=0.05 \mathrm{~cm} \mathrm{~s}^{-1}, E_{2}^{0}=0.195 \mathrm{~V}$ (vs. Fc/Fc $\left.{ }^{+}\right), k_{2}^{0}=0.08 \mathrm{~cm} \mathrm{~s}^{-1}, \alpha=0.5 . D($ TEMPO/oxoammonium) $=2.2 \times 10^{-5} \mathrm{~cm}^{2} \mathrm{~s}^{-1}, D\left(3 / 3^{++}\right)=7.8 \times 10^{-6} \mathrm{~cm}^{2} \mathrm{~s}^{-1}$ and for the irreversible chemical step $k_{f}$ is $5.0 \times 10^{6} \mathrm{~s}^{-1}$. Only the first 4 segments are shown for clarity and the first segment is acquired ramping the bias in the anodic direction. The electrochemically-determined effective area for the data in figure is $0.07 \mathrm{~cm}^{2}$. An estimate of the $\mathrm{Pt}$ macrodisk effective area was obtained prior to each experiment on 3 by refining cyclic voltammograms of $0.5 \times 10^{-3} \mathrm{M} \mathrm{FC}$ in $\mathrm{MeCN}$ with $1.0 \times 10^{-1} \mathrm{M} \mathrm{Bu}_{4} \mathrm{NClO}_{4}$ and using the following model: $\mathrm{E}$ mechanism, $E^{0}\left(\mathrm{Fc} / \mathrm{Fc}^{+}\right)=0.23 \mathrm{~V}(\mathrm{vs}$. $\mathrm{Ag} / \mathrm{AgCl}), K^{0}\left(\mathrm{Fc} / \mathrm{Fc}^{+}\right)=0.2 \mathrm{~cm} \mathrm{~s}^{-1}, D\left(\mathrm{Fc} / \mathrm{Fc}^{+}\right)=2.3 \times 10^{-5} \mathrm{~cm}^{2} \mathrm{~s}^{-1}, \alpha=0.5$. 

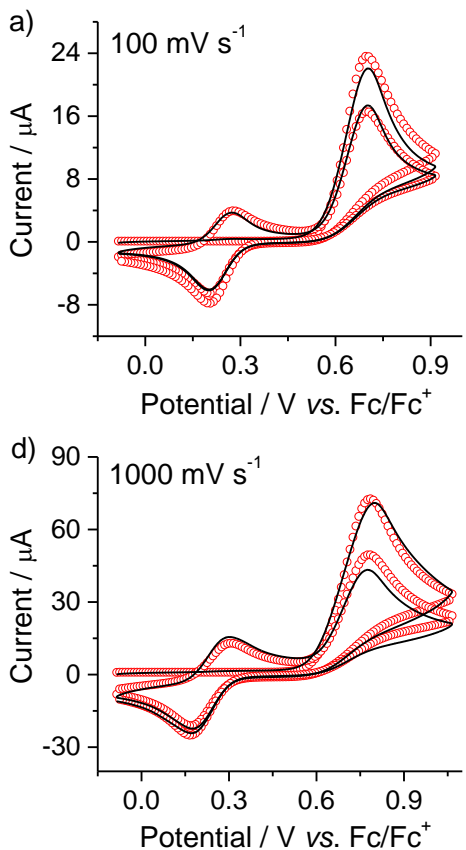

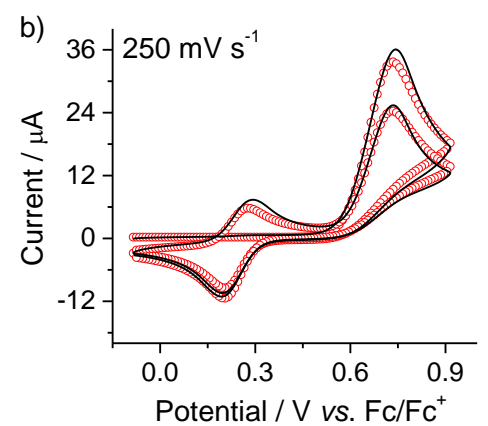

e)

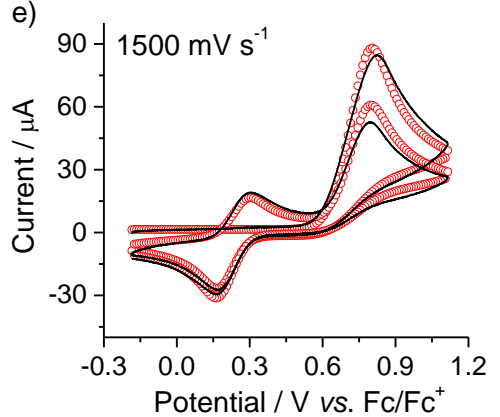

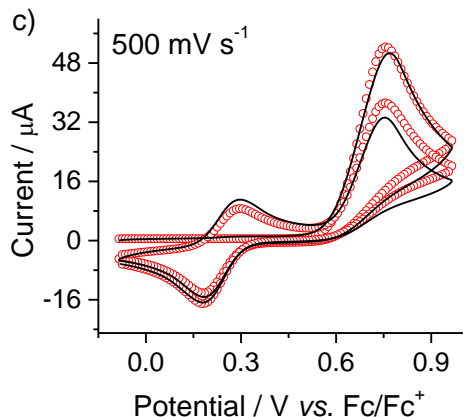

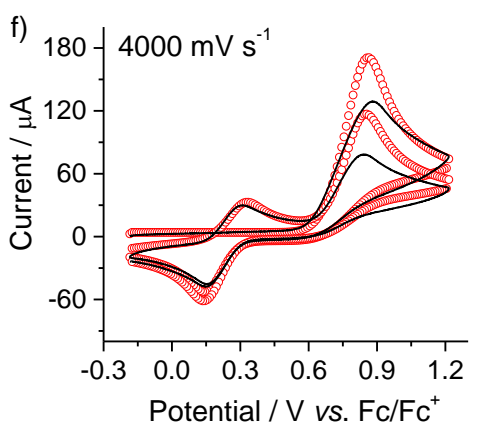

Figure S6. Anodic electrochemistry of alkoxyamine 3. Experimental (solid line) and simulated (empty symbols) voltammograms at different scan rates (100 to $4000 \mathrm{mV} \mathrm{s}^{-1}$, as specified by labels in figure) of $5.0 \times 10^{-3} \mathrm{M} 3$ in MeCN with $1.0 \times 10^{-1} \mathrm{M} \mathrm{Bu}_{4} \mathrm{NClO}_{4}$. Best-fit parameters $\left(\mathrm{EC}_{\text {irr }} \mathrm{E}\right.$ mechanism) are: $E_{1}^{0}=0.83 \mathrm{~V}$ (vs. $\left.\mathrm{Fc} / \mathrm{Fc}^{+}\right), k_{1}^{0}=0.06 \mathrm{~cm} \mathrm{~s}{ }^{-1}, E_{2}^{0}=0.245 \mathrm{~V}\left(\mathrm{vs} . \quad \mathrm{Fc} / \mathrm{Fc}^{+}\right), k_{2}^{0}=0.02 \mathrm{~cm} \mathrm{~s}^{-1}, \alpha=0.5 . \quad D$ (TEMPO/oxoammonium) $=2.2 \times 10^{-5} \mathrm{~cm}^{2} \mathrm{~s}^{-1}, D\left(3 / 3^{++}\right)=7.8 \times 10^{-6} \mathrm{~cm}^{2} \mathrm{~s}^{-1}$ and for the irreversible chemical step $k_{f}$ is $5.0 \times 10^{6} \mathrm{~s}^{-1}$. The electrochemically-determined effective area for the data in figure is $0.017 \mathrm{~cm}^{2}$. Only the first 4 segments are shown for clarity and the first segment is acquired ramping the bias in the anodic direction. 


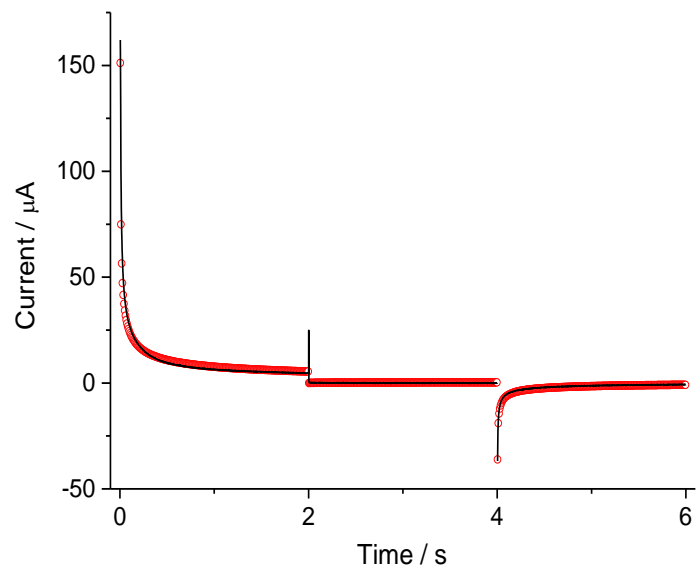

Figure S7. Current evolution with time in a typical three-step chronoamperometry experiment of $0.36 \times 10^{-3} \mathrm{M} 3$ in MeCN with $1.0 \times 10^{-1} \mathrm{M}$ $\mathrm{Bu}_{4} \mathrm{NClO}_{4}$. Experimental (solid line) and simulated (symbols) transients with the potential being stepped from $-0.03 \mathrm{~V}$ to $0.87 \mathrm{~V}$, then from 0.87 $\mathrm{V}$ to $0.47 \mathrm{~V}$, and from $0.47 \mathrm{~V}$ to $-0.03 \mathrm{~V}\left(\mathrm{vs}\right.$. $\left.\mathrm{Fc} / \mathrm{Fc}^{+}\right)$. The best-fit parameters are $D\left(\right.$ TEMPO/oxoammonium) $=2.2 \times 10^{-5} \mathrm{~cm}^{2} \mathrm{~s}^{-1}, D\left(3 / 3^{-4}\right)=7.8$ $\times 10^{-6} \mathrm{~cm}^{2} \mathrm{~s}^{-1} E_{1}^{0}=0.78 \vee\left(\mathrm{vs} . \mathrm{Fc} / \mathrm{Fc}^{+}\right), k_{1}^{0}=0.05 \mathrm{~cm} \mathrm{~s}^{-1}, E_{2}^{0}=0.195 \vee\left(\mathrm{vs} . \mathrm{Fc} / \mathrm{Fc}^{+}\right), k_{2}^{0}=0.08 \mathrm{~cm} \mathrm{~s}^{-1}, k_{f}=5.0 \times 10^{6} \mathrm{~s}^{-1}$. The electrochemically-determined effective area for the data in figure is $0.07 \mathrm{~cm}^{2}$. 

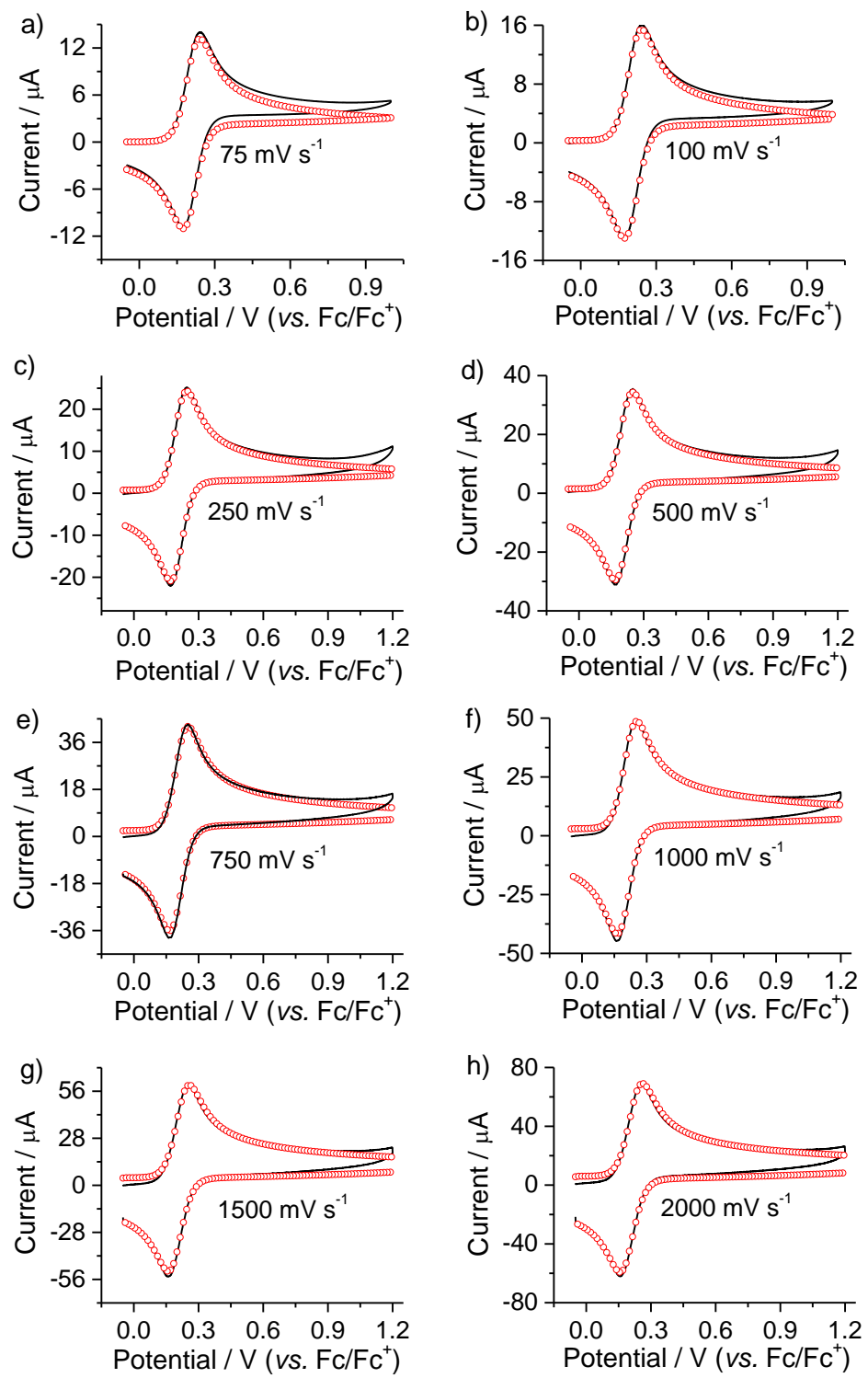

Figure S8. Experimental (solid line) and simulated (empty symbol) cyclic voltammograms for $0.5 \times 10^{-3} \mathrm{M}$ TEMPO solutions in MeCN containing $1.0 \times 10^{-1} \mathrm{M} \mathrm{Bu}_{4} \mathrm{NClO}_{4}$ at different scan rates (indicated as labels to the figures). The diffusion coefficients and the electron transfer rate constant used to fit the data were $2.2 \times 10^{-5} \mathrm{~cm}^{2} \mathrm{~s}^{-1}\left(D_{T E M P O}\right), 2.2 \times 10^{-5} \mathrm{~cm}^{2} \mathrm{~s}^{-1}\left(D_{\text {oxoammonium }}\right)$ and $0.08 \mathrm{~cm} \mathrm{~s}^{-1}\left(k_{T E M P O}^{0}\right), E_{T E M P O}^{0}=0.21 \mathrm{~V}$ (vs. $\mathrm{Fc} / \mathrm{Fc}^{+}$). The electrochemically-determined effective area of the Pt disk electrode is $0.07 \mathrm{~cm}^{2}$. 

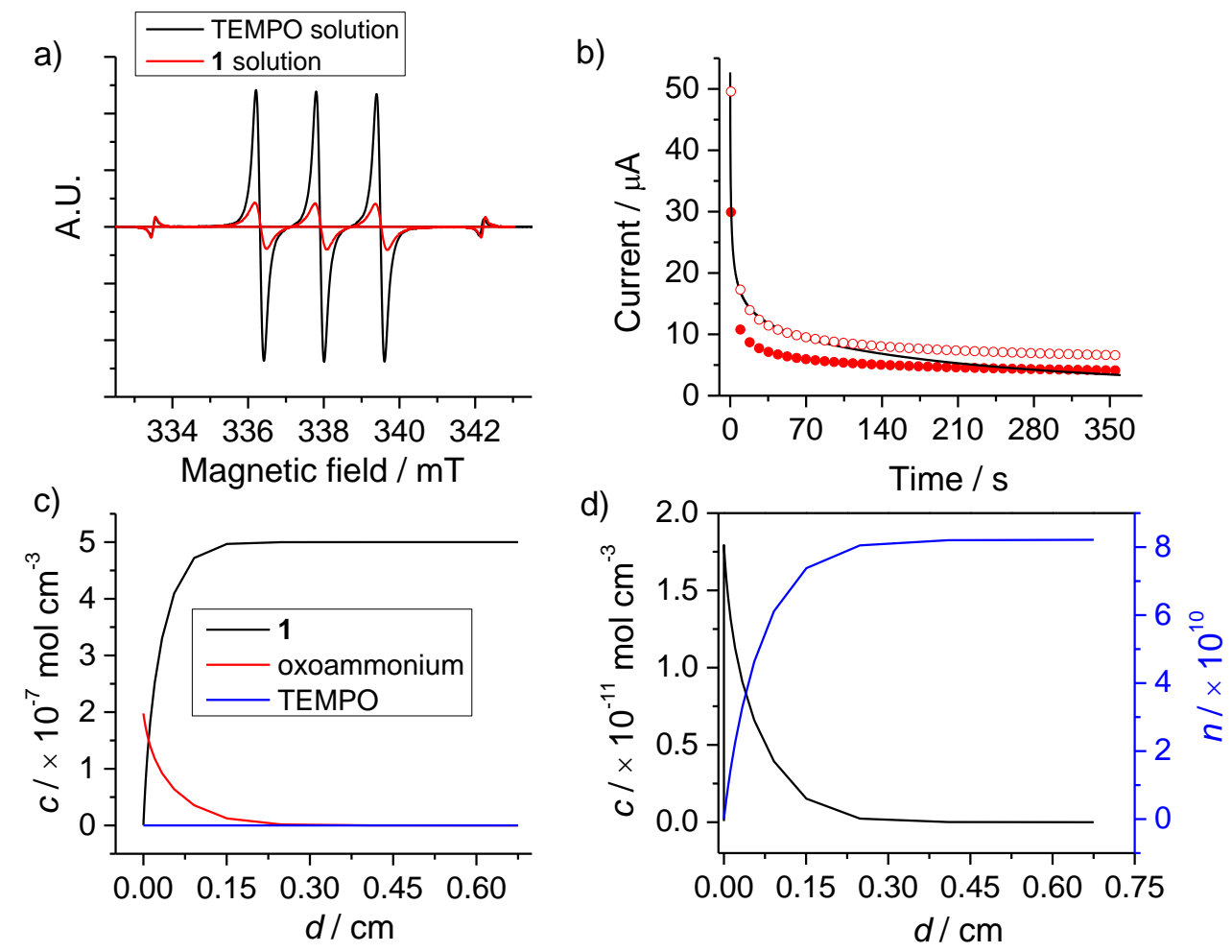

Figure S9. (a) EPR spectra (red trace) of anodized TEMPO-PE solutions $\left(0.5 \times 10^{-3} \mathrm{M} 3\right.$ in DCM with $\left.1.0 \times 10^{-1} \mathrm{M} \mathrm{Bu}_{4} \mathrm{NPF}_{6}\right)$. The working Pt bias is poised to $0.85 \mathrm{~V}$ vs. $\mathrm{Fc} / \mathrm{Fc}^{+}$, the anodization time is $360 \mathrm{~s}$ and the EPR spectra is accumulated during the last $60 \mathrm{~s}$ of the anodic pulse. The EPR spectra of $\mathbf{3}$ is plotted against the EPR spectra (acquired at OCP) of control TEMPO solutions (black trace). (b) Experimental (solid line) and simulated (symbols) current-time transients after a potential step from OCP to $0.85 \mathrm{~V}$ ( $\mathrm{vs}$. Fc/ $\mathrm{Fc}^{+}$, cylindrical geometry, $0.01 \mathrm{~cm}$ radius and length of either $2.5 \mathrm{~cm}$ (solid symbol) or $4 \mathrm{~cm}$ (empty symbol), and semi-infinite 1D diffusion). The electrolyte is a $0.5 \times 10^{-3} \mathrm{M}$ solution of 3 and for the simulations (symbols) we considered an $\mathrm{EC}_{\text {irr }} \mathrm{E}$ mechanism that is described by the following parameters: $E_{1}^{0}=0.80 \mathrm{~V}\left(\mathrm{vs}\right.$. $\left.\mathrm{Fc} / \mathrm{Fc}^{+}\right)$, $k_{1}^{0}=0.01 \mathrm{~cm} \mathrm{~s}^{-1}, E_{2}^{0}=0.30 \mathrm{~V}, k_{2}^{0}=0.01 \mathrm{~cm} \mathrm{~s}^{-1}, \alpha=0.5 . D$ (TEMPO/oxoammonium) $=1.9 \times 10^{-5} \mathrm{~cm}^{2} \mathrm{~s}^{-1}, D\left(3 / 3^{++}\right)=6.0 \times 10^{-6} \mathrm{~cm}^{2} \mathrm{~s}^{-1} . \mathrm{The}^{\circ}$ value of $k_{f}$ for the irreversible chemical step is $1.0 \times 10^{6} \mathrm{~s}^{-1}$. (c) Simulated concentration profiles (3, oxoammonium and TEMPO, EC irrE parameters as at panel (b)) near the cylindrical Pt wire $(0.01 \mathrm{~cm}$ radius and $2.5 \mathrm{~cm}$ in length) and (d) simulated cumulative number of TEMPO molecules (right $y$-axis) present in the EPR sample after the $360 \mathrm{~s}$ potential step.

As seen from panel (a) in Figure S9, the EPR signal emerging during the anodization of 3 ( $0.5 \mathrm{mM}$ solutions, $0.4 \mathrm{~mL}$ total sample volume) is in agreement with pure the EPR signal of a TEMPO control solution. The standard based on $\mathrm{Mn}^{2+}$ was used to calibrate g-factor. EPR spectra of $\mathrm{Mn}^{2+}$ consists of six spectral lines. Organic radicals absorb energy in the range of magnetic field between the third and the fourth spectral line of $\mathrm{Mn}^{2+}$. In order to calculate $\mathrm{g}$-factor, the spectra of $\mathrm{Mn}^{2+}$ (the third and fourth line) together with investigated sample was measured. Based on known values of $\mathrm{g}$-factor of corresponding lines of $\mathrm{Mn}^{2+}$ spectra (2.03277 for $3^{\text {rd }}$ and 1.98104 for $4^{\text {th }}$ spectral line) the g-factor of investigated sample was calculated. G-factor of pure TEMPO as well as $\mathbf{3}^{\mathbf{}}$ is equal to 2.00619 (calculated for the center of spectra). In order to get more insight into cumulative number of TEMPO molecules from electrode surface to bulk solution, we simulated the concentration profiles of the different species involved and generated during the anodization of 3 (panel (c), Figure S9). As illustrated in panel (d), the estimated number of spins (cumulative number of TEMPO molecules after $360 \mathrm{~s}$ of sample anodization) is in line with the detection limit of nitroxide radicals by $\mathrm{EPR}^{13}$. 

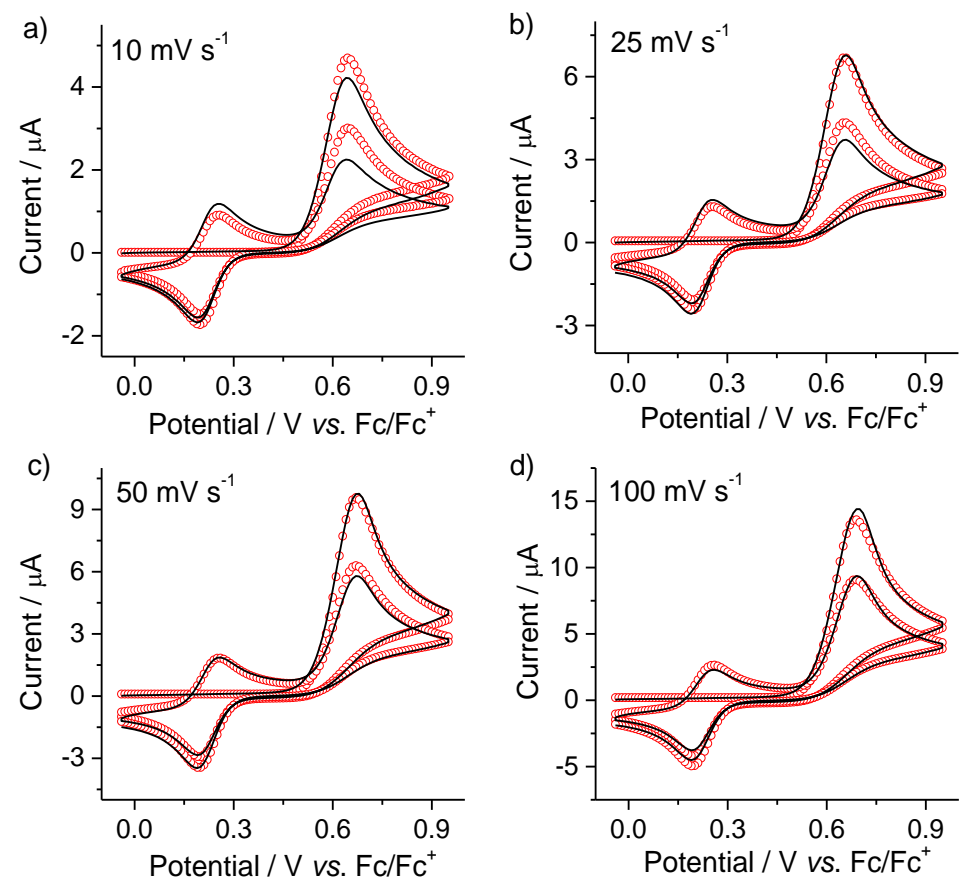

Figure S10. Anodic electrochemistry of alkoxyamine 3. Experimental (solid line) and simulated (empty symbols) voltammograms at different

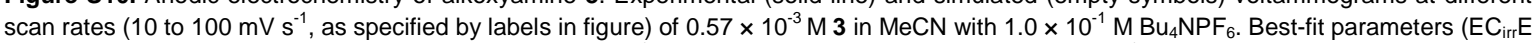
mechanism) are: $E_{1}^{0}=0.81 \mathrm{~V}$ (vs. Fc/Fc $\left.{ }^{+}\right), k_{1}^{0}=0.08 \mathrm{~cm} \mathrm{~s}^{-1}, E_{2}^{0}=0.225 \mathrm{~V}$ (vs. Fc/Fc $\left.{ }^{+}\right), k_{2}^{0}=0.04 \mathrm{~cm} \mathrm{~s}^{-1}, \alpha=0.5 . D($ TEMPO/oxoammonium) $=2.2 \times 10^{-5} \mathrm{~cm}^{2} \mathrm{~s}^{-1}, D\left(3 / 3^{++}\right)=7.7 \times 10^{-6} \mathrm{~cm}^{2} \mathrm{~s}^{-1}$ and for the irreversible chemical step $k_{f}$ is $5.0 \times 10^{6} \mathrm{~s}^{-1}$. The electrochemically-determined effective area for the data in figure is $0.08 \mathrm{~cm}^{2}$. Only the first 4 segments are shown for clarity and the first segment is acquired ramping the bias in the anodic direction. 


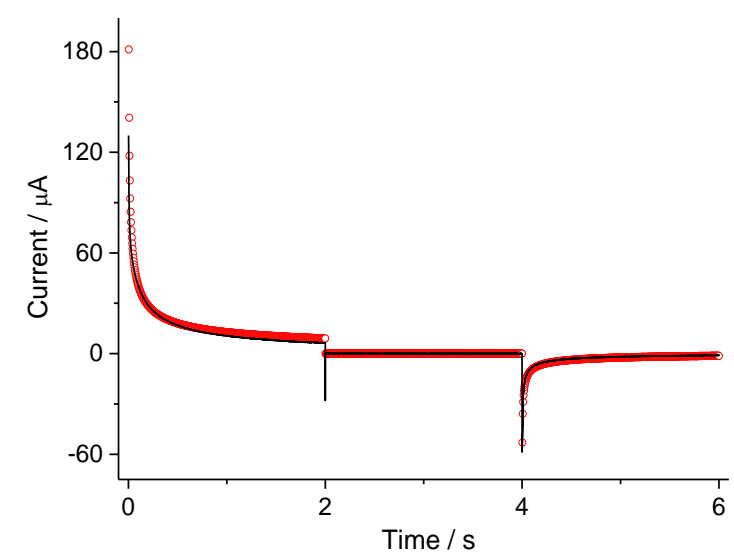

Figure S11. Current evolution with time in a typical three-step chronoamperometry experiment of $0.6 \times 10^{-3} \mathrm{M} 3$ in MeCN with $1.0 \times 10^{-1} \mathrm{M}$ $\mathrm{Bu}_{4} \mathrm{NPF}_{6}$. Experimental (solid line) and simulated (symbols) transients with the potential being stepped from $-0.04 \mathrm{~V}$ to $0.86 \mathrm{~V}$, then from $0.86 \mathrm{~V}$ to $0.46 \mathrm{~V}$, and from $0.46 \mathrm{~V}$ to $-0.04 \mathrm{~V}\left(\mathrm{vs}\right.$. $\left.\mathrm{Fc} / \mathrm{Fc}^{+}\right)$. The electrochemically-determined effective area for the data in figure is $0.07 \mathrm{~cm}^{2}$. The best-fit parameters are $D$ (TEMPO/oxoammonium) $=2.2 \times 10^{-5} \mathrm{~cm}^{2} \mathrm{~s}^{-1}, D\left(3 / 3^{++}\right)=7.7 \times 10^{-6} \mathrm{~cm}^{2} \mathrm{~s}^{-1} E_{1}^{0}=0.81 \mathrm{~V}(\mathrm{vs}$. Fc/Fc $), k_{1}^{0}=0.08 \mathrm{~cm} \mathrm{~s}^{-1}, E_{2}^{0}=$ $0.225 \mathrm{~V}\left(\mathrm{vs} . \mathrm{Fc} / \mathrm{Fc}^{+}\right), k_{2}^{0}=0.04 \mathrm{~cm} \mathrm{~s}^{-1}, k_{f}=5.0 \times 10^{6} \mathrm{~s}^{-1}$. 


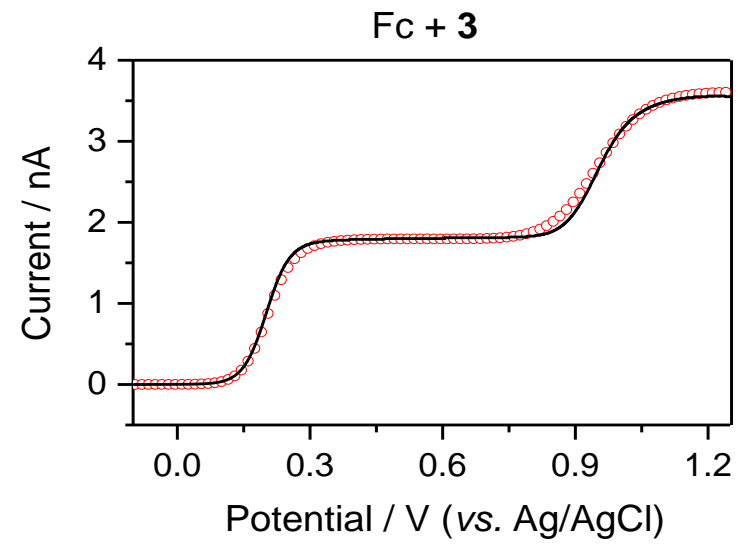

Figure S12. Cyclic voltammograms at Pt microdisks $\left(5.0 \mu \mathrm{m}\right.$ in radius) of $0.50 \times 10^{-3} \mathrm{M} \mathrm{FC}$ and $0.64 \times 10^{-3} \mathrm{M} 3$ in $\mathrm{MeCN}$ with $1.0 \times 10^{-2}$ $\operatorname{NaBARF}$ (scan rate is $\left.10 \mathrm{mV} \mathrm{s}^{-1}\right)$. The refined parameters $\left(\mathrm{EC}_{\text {irr }} \mathrm{E}\right.$ mechanism) are: $\mathrm{E}^{0}\left(\mathrm{Fc} / \mathrm{Fc}^{+}\right)=0.20 \mathrm{~V} \mathrm{vs} \mathrm{Ag} / \mathrm{AgCl}$ ("leakless"), $k^{0}\left(\mathrm{Fc} / \mathrm{Fc}^{+}\right)=$ $0.2 \mathrm{~cm} \mathrm{~s}^{-1}, E^{0}\left(3 / 3^{*+}\right)=1.025 \mathrm{~V}, k^{0}\left(3 / 3^{\cdot+}\right)=0.07 \mathrm{~cm} \mathrm{~s}^{-1}, E^{0}($ TEMPO/oxoammonium $)=0.435 \mathrm{~V}, k^{0}($ TEMPO/oxoammonium $)=0.05 \mathrm{~cm} \mathrm{~s}^{-1}, \alpha=$ 0.5 . For the chemical step of $3^{*+} \rightarrow$ TEMPO $+\mathrm{R}^{+}$, the refined $k_{\mathrm{f}}$ is $5.2 \times 10^{6} \mathrm{~s}^{-1}$. The experimental and simulated curves are plotted as solid lines and empty symbols, respectively. The diffusivity of $\mathrm{Fc}$ is set to $1.8 \times 10^{-5} \mathrm{~cm}^{2} \mathrm{~s}^{-1}$, that for the TEMPO/oxoammonium couple is $2.0 \times 10^{-5} \mathrm{~cm}^{2} \mathrm{~s}^{-}$ and the diffusivity of the $3 / 3^{++}$couple is $7.3 \times 10^{-6} \mathrm{~cm}^{2} \mathrm{~s}^{-1}$. 


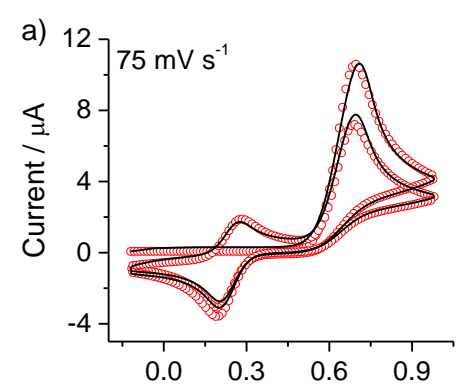

d)
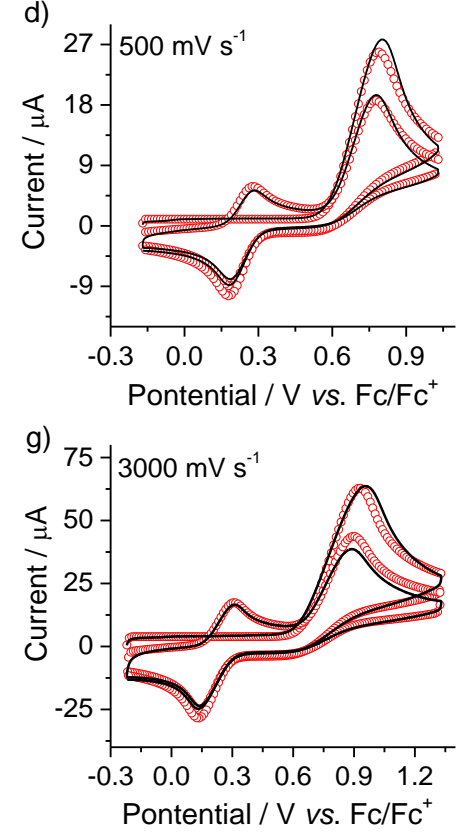

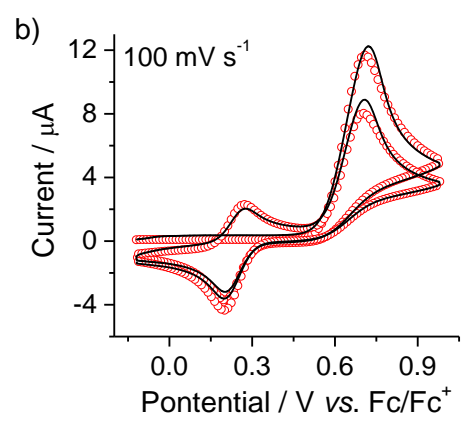

e)

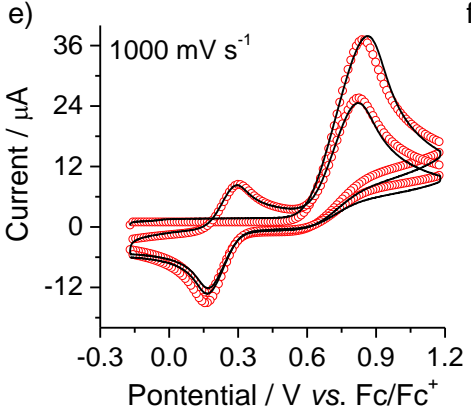

h)

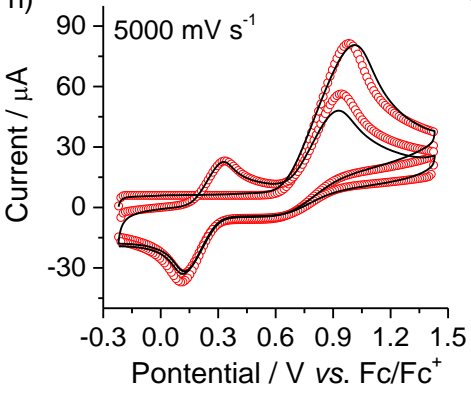

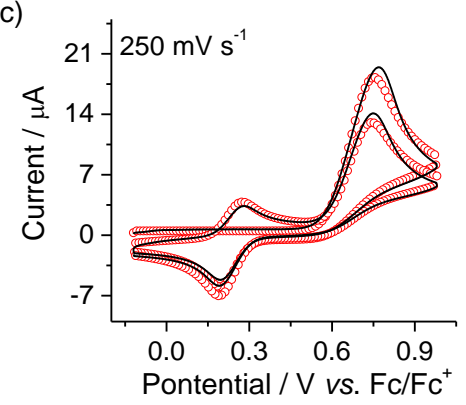

f)
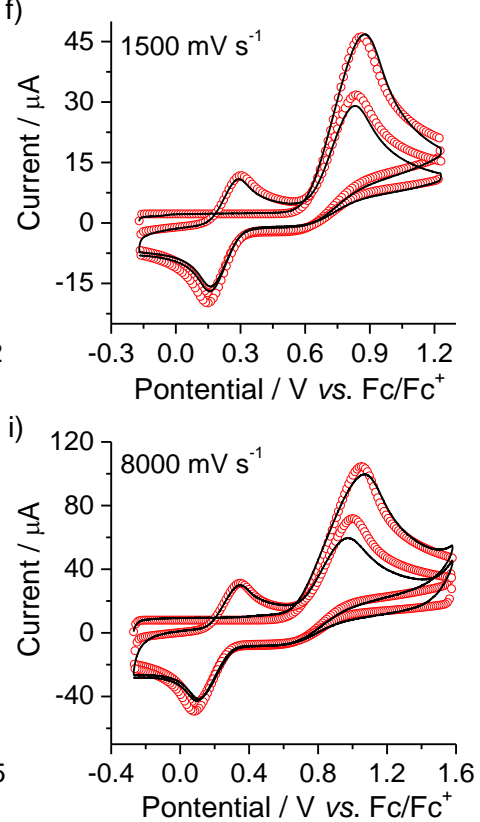

Figure S13. Anodic electrochemistry of alkoxyamine 3. Experimental (solid line) and simulated (empty symbols) voltammograms at different

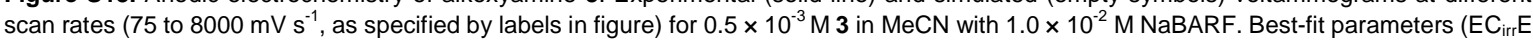
mechanism) are: $E_{1}^{0}=0.82 \mathrm{~V}$ (vs. Fc/Fc $\left.{ }^{+}\right), k_{1}^{0}=0.07 \mathrm{~cm} \mathrm{~s}^{-1}, E_{2}^{0}=0.23 \mathrm{~V}$ (vs. Fc/Fc $\left.{ }^{+}\right), k_{2}^{0}=0.05 \mathrm{~cm} \mathrm{~s}^{-1}, \alpha=0.5 D($ TEMPO/oxoammonium). = $2.0 \times 10^{-5} \mathrm{~cm}^{2} \mathrm{~s}^{-1}, D\left(\mathbf{3} / 3^{-+}\right)=7.3 \times 10^{-6} \mathrm{~cm}^{2} \mathrm{~s}^{-1}$ and for the irreversible chemical step $k_{f}$ is $5.2 \times 10^{6} \mathrm{~s}^{-1}$. The electrochemically-determined effective area for the data in figure is $0.07 \mathrm{~cm}^{2}$. Only the first 4 segments are shown for clarity and the first segment is acquired ramping the bias in the anodic direction. 


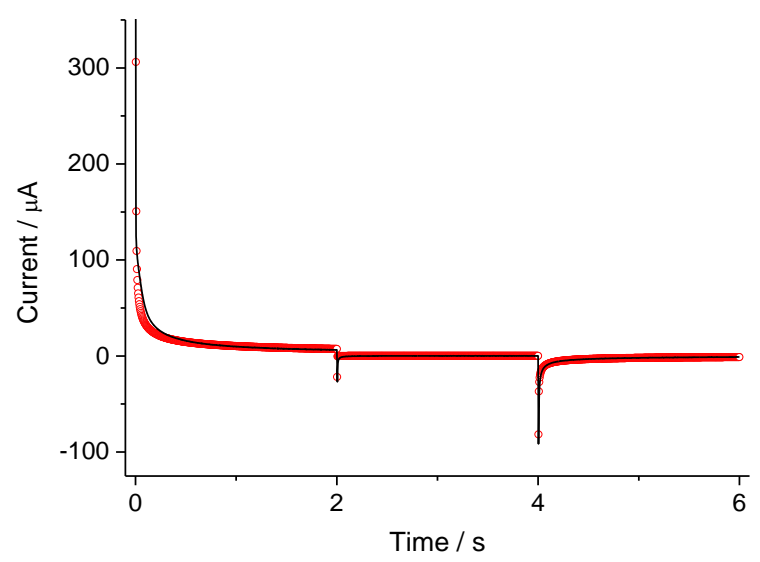

Figure S14. Current evolution with time in a typical three-step chronoamperometry experiment of $0.5 \times 10^{-3} \mathrm{M} 3$ in MeCN with $1.0 \times 10^{-2} \mathrm{M}$ NaBARF. Experimental (solid line) and simulated (symbols) transients with the potential being stepped from $-0.04 \mathrm{~V}$ to $1.06 \mathrm{~V}$, then from $1.06 \mathrm{~V}$ to $0.46 \mathrm{~V}$, and from $0.46 \mathrm{~V}$ to $-0.04 \mathrm{~V}\left(\mathrm{vs}\right.$. Fc/ $\left.\mathrm{Fc}^{+}\right)$. The electrochemically-determined effective area for the data in figure is $0.07 \mathrm{~cm}^{2}$. The best-fit parameters are $D$ (TEMPO/oxoammonium) $=2.0 \times 10^{-5} \mathrm{~cm}^{2} \mathrm{~s}^{-1}, D\left(3 / 3^{-+}\right)=7.3 \times 10^{-6} \mathrm{~cm}^{2} \mathrm{~s}^{-1} E_{1}^{0}=0.82 \mathrm{~V}(\mathrm{vs}$. Fc/Fc $), k_{1}^{0}=0.07 \mathrm{~cm} \mathrm{~s}^{-1}, E_{2}^{0}=$ $0.23 \mathrm{~V}\left(\mathrm{vs} . \mathrm{Fc} / \mathrm{Fc}^{+}\right), k_{2}^{0}=0.05 \mathrm{~cm} \mathrm{~s}^{-1}, k_{f}=5.2 \times 10^{6} \mathrm{~s}^{-1}$. 

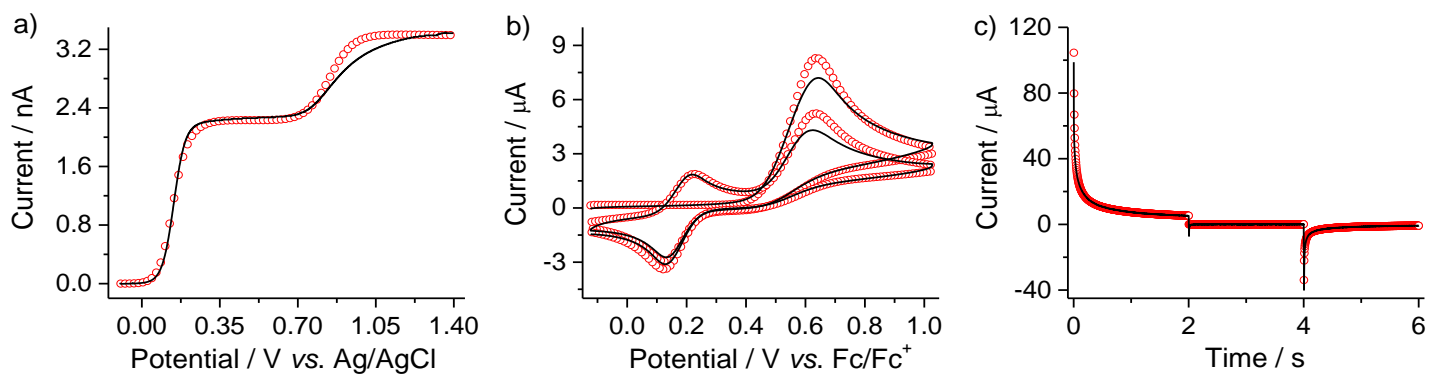

Figure S15. (a-b) Anodic electrochemistry of alkoxyamine 3 in DCM. Experimental (solid line) and simulated (empty symbols) voltammograms of $0.5 \times 10^{-3} \mathrm{M} 3$ in DCM with $1.0 \times 10^{-1} \mathrm{M} \mathrm{Bu}_{4} \mathrm{NPF}_{6}\left(50 \mathrm{mV} \mathrm{s}^{-1}\right.$ for Pt ultramicroelectrodes $5.0 \mu \mathrm{m}$ in radius in (a), the electrolyte is added with $0.6 \times 10^{-3} \mathrm{M}$ Fc. The refined parameters $\left(\mathrm{EC}_{\text {irr }} \mathrm{E}\right.$ mechanism) are: $E^{0}\left(\mathrm{Fc} / \mathrm{Fc}^{+}\right)=0.13 \mathrm{~V} \mathrm{vs} \mathrm{Ag} / \mathrm{AgCl}$ ("leakless"), $k^{0}\left(\mathrm{Fc} / \mathrm{Fc}^{+}\right)=0.10 \mathrm{~cm} \mathrm{~s}{ }^{-1}, E^{0}$ $\left(3 / 3^{*+}\right)=0.82 \mathrm{~V}, k^{0}\left(3 / 3^{++}\right)=0.01 \mathrm{~cm} \mathrm{~s}^{-1}, E^{0}$ (TEMPO/oxoammonium) $=0.32 \mathrm{~V}, k^{0}$ (TEMPO/oxoammonium) $=0.01 \mathrm{~cm} \mathrm{~s}{ }^{-1}, \alpha=0.5$. For the chemical step of $3^{-+} \rightarrow$ TEMPO $+R^{+}$, the refined $k_{\mathrm{f}}$ is $1.0 \times 10^{6} \mathrm{~s}^{-1}$. The diffusivity of Fc was set equal to $1.9 \times 10^{-5} \mathrm{~cm}^{2} \mathrm{~s}^{-1}$. The diffusivity of the $3 / 3^{++}$couple was $6.0 \times 10^{-6} \mathrm{~cm}^{2} \mathrm{~s}^{-1}$. The refined $D$ value of $1.9 \times 10^{-5} \mathrm{~cm}^{2} \mathrm{~s}^{-1}$ was for the TEMPO/oxoammonium couple; $50 \mathrm{mV} \mathrm{s}{ }^{-1}$ for $0.07 \mathrm{~cm}^{2}$ Pt macro-disks electrodes presented in (b)). Best-fit parameters $\left(\mathrm{EC}_{\text {irr }} \mathrm{E}\right.$ mechanism) are: $E_{1}^{0}=0.68 \mathrm{~V}\left(\mathrm{vs} . \mathrm{Fc} / \mathrm{Fc}^{+}\right), k_{1}^{0}=0.01 \mathrm{~cm} \mathrm{~s}^{-1}, E_{2}^{0}=0.18$ $\mathrm{V}\left(\mathrm{vs}\right.$. Fc/Fc $\left.{ }^{+}\right), \quad k_{2}^{0}=0.01 \mathrm{~cm} \mathrm{~s}^{-1}, \alpha=0.5 . D$ (TEMPO/oxoammonium) $=1.9 \times 10^{-5} \mathrm{~cm}^{2} \mathrm{~s}^{-1}, D\left(3 / 3^{-+}\right)=6.0 \times 10^{-6} \mathrm{~cm}^{2} \mathrm{~s}^{-1}$ and for the irreversible chemical step $k_{f}$ is $1.0 \times 10^{6} \mathrm{~s}^{-1}$. (c) Current evolution with time in a typical three-step chronoamperometry experiment of $0.4 \times 10^{-3} \mathrm{M} 3$ in $\mathrm{DCM}$ with $1.0 \times 10^{-1} \mathrm{M} \mathrm{Bu}_{4} \mathrm{NPF}_{6}$. Experimental (solid line) and simulated (symbols) transients with the potential being stepped from $-0.07 \mathrm{~V}$ to $0.78 \mathrm{~V}$, then from $0.78 \mathrm{~V}$ to $0.43 \mathrm{~V}$, and from $0.43 \mathrm{~V}$ to $-0.07 \mathrm{~V}$ (vs. $\left.\mathrm{Fc} / \mathrm{Fc}^{+}\right)$. The best-fit parameters are $D\left(\right.$ TEMPO/oxoammonium) $=1.9 \times 10^{-5}$ $\mathrm{cm}^{2} \mathrm{~s}^{-1}, D\left(3 / 3^{++}\right)=6.0 \times 10^{-6} \mathrm{~cm}^{2} \mathrm{~s}^{-1} E_{1}^{0}=0.69 \mathrm{~V}\left(\mathrm{vs} . \mathrm{Fc} / \mathrm{Fc}^{+}\right), k_{1}^{0}=0.01 \mathrm{~cm} \mathrm{~s}^{-1}, E_{2}^{0}=0.19 \mathrm{~V}\left(\mathrm{vs} . \mathrm{Fc} / \mathrm{Fc}^{+}\right), k_{2}^{0}=0.01 \mathrm{~cm} \mathrm{~s}^{-1}, k_{f}=1.0 \times 10^{6} \mathrm{~s}^{-1}$. The electrochemically-determined effective area for the data in figure is $0.07 \mathrm{~cm}^{2}$. We remark that the refinement of a model against the experimental voltammetry of $\mathbf{3}$ in DCM-based electrolytes was generally found to lead to poorer quality fits relative to the MeCN counterparts. 


\section{S7. Theoretical study of the effect of external electric field on alkoxyamine cleavage}

Theoretical Methodology. To examine the effect of external electric fields (EEFs) on the bond cleavage reaction of alkoxyamines, the reaction energy of the C-O bond cleavage reaction in Scheme S3 was calculated as a function of field strength. This model includes the tethers which would attach to the gold surfaces in the STM experiment, however the gold itself isn't included due to the large increase in computational cost and complexity associated with dealing with modelling a surface. Moreover, previous studies suggest that the gold cluster in that case underestimated the effect of EEFs by more than a third, so they will not be considered for this study. ${ }^{14}$ In addition to the preferred homolytic cleavage reaction, two alternative heterolytic cleavage reactions were also studied as a function of field strength. These calculations confirmed that homolytic cleavage remained the preferred process across all field strengths studied. Calculations were performed in both the gas phase and in mesitylene solvent, using the SMD solvent model, to mimic experimental conditions. All calculations were performed using Gaussian $09^{15}$ with $M 06-2 X / 6-31+G(d)$ as the level of theory, which has been benchmarked and used in previous studies of EEFs. The electronic energies are used in the results as the entropic calculation in an STM experiment is difficult to quantify and in any case these contributions are expected to be relatively independent of field strength and cancel. ${ }^{14}$ Full conformer searching and geometry relaxation of each species was performed and the lowest energy conformer then used for subsequent calculations involving fields.

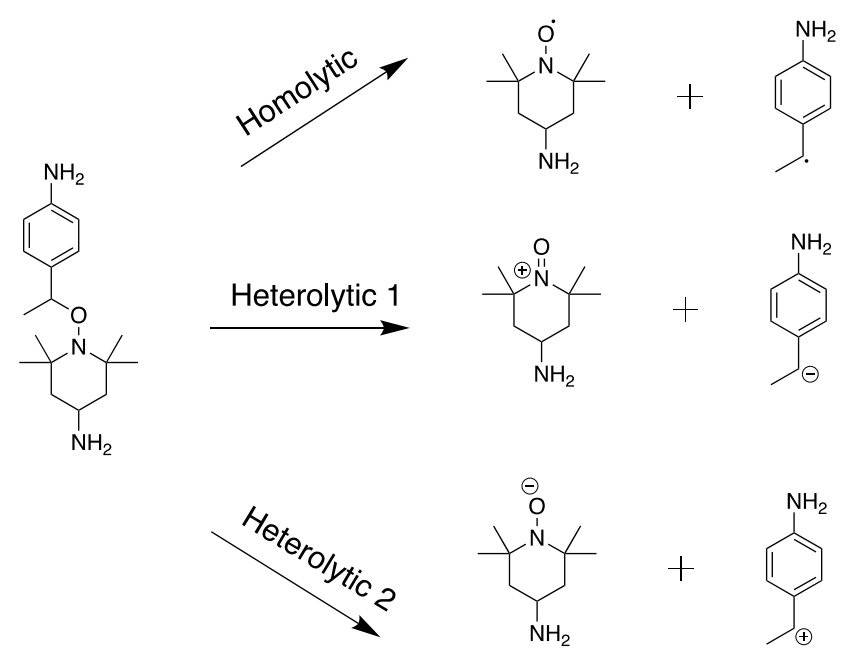

Scheme S3. Alkoxyamine homolytic and heterolytic bond cleavage

In assessing the effects of EEFs, the field strength and direction needs to be chosen so as to mimic the experimental conditions. This is more complex than in our previous study of Diels-Alder reactions ${ }^{14}$ in EEFs for two reasons: (1) the alkoxyamine is tethered but not as held rigidly in the STM as the norbornene derivatives of the previous work; (2) the present experiments are STM tapping experiments rather than blinking. In the latter, the tip is held a fixed distance above the plate and this distance, together with the applied voltage, can be used to calculate the field strength experienced by the reagents. In tapping experiments, the tip is repeatedly driven into the plate and in principle the field at any applied voltage is variable can reach very levels as the distance between the tip and the plate diminish. Given these problems it was decided to assess EEFs along a wide range of field strengths, +0.0125 au to -0.0125 au, a range chosen for consistency with previous theoretical studies of the effects of EEFs ${ }^{16}$ on chemical reactions. It is presumed that if the molecule is free to move to some extent in the field then it should, within the constraints of tethering, adopt the orientation most likely to minimize its energy, both in the reactant and product. Thus the field was initially aligned along the reaction axis (the Z-axis in Figure S16 below), but additional sensitivity analysis was also undertaken using moderate strengths of +0.0010 au (approximately $0.5 \mathrm{~V} \mathrm{~nm}^{-1}$ ). In performing these calculations, rotation of the coordinate axis was prevented using the Nosymm keyword and the field itself was applied using the Field = Read keyword. At every field strength, the geometries were allowed to relax to obtain the lowest energy of each species at every field strength tested. 

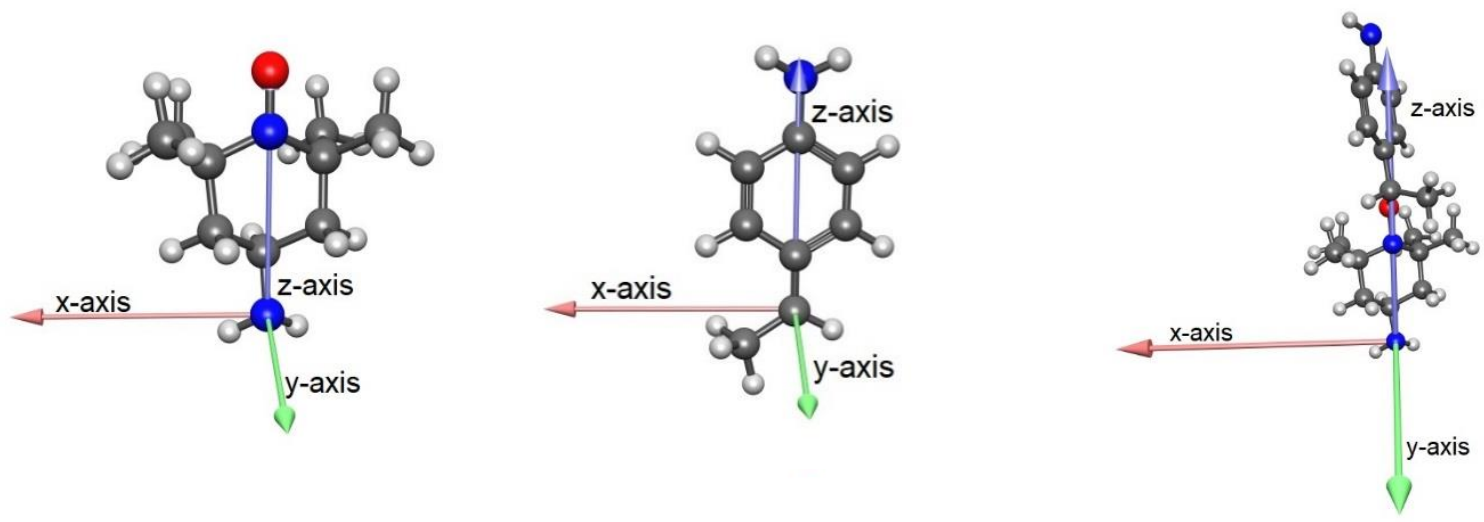

Figure S16. Selection of the $\mathrm{x}-, \mathrm{y}$ - and z-directions (axes) for the reactant and product molecules of the homolytic cleavage reaction.

Theoretical Results. Figure S17 shows the effect of the oriented EEF on the reaction energy for homolytic cleavage as a function of field strength and bias in both the gas phase and mesitylene solvent. The effects of solvent are relatively minor except at very high negative biases. This is likely to be unphysical and resulting from the problems associated with using a continuum solvent model (in which the solvent is effectively modelled as an electric field) in an external electric field. Unfortunately modelling a large cluster of explicit solvent molecules at an appropriate level of theory is currently impractical. This aside, it is obvious that field can significantly affect the reaction, increasing the homolysis reaction energy at negative bias and facilitating it at positive bias. This effect has been shown previously using charged functional groups and point charges, ${ }^{17}$ and this arises from the fact that the nitroxide radical product of homolysis is stabilized by resonance with a charge separated contributor $\left(\mathrm{N}-\mathrm{O}^{\circ} \leftrightarrow \mathrm{N}^{+\cdot}-\mathrm{O}^{-}\right)$. An appropriately aligned field can thus electrostatically stabilize or destabilise the dipole associated with this resonance contributor and in doing so stabilize or destabilize the products of homolysis. To put the results into a practical perspective, at the maximum positive bias studied, promotion of homolysis by ca. $35 \mathrm{~kJ} / \mathrm{mol}$ would increase the homolysis equilibrium constant by approximately 6 orders of magnitude at room temperature to ca. $10^{-11}$. This, for example, produces more than enough radicals to sustain a controlled nitroxide mediated polymerization. ${ }^{18}$ Moreover, the local fields experienced as the tip crashes into the plate are likely to be much higher than this, leading to greater homolysis. Whilst the modelling is by necessity approximate, it is clear that fields effects can in principle promote homolysis and explain the observed STM results.

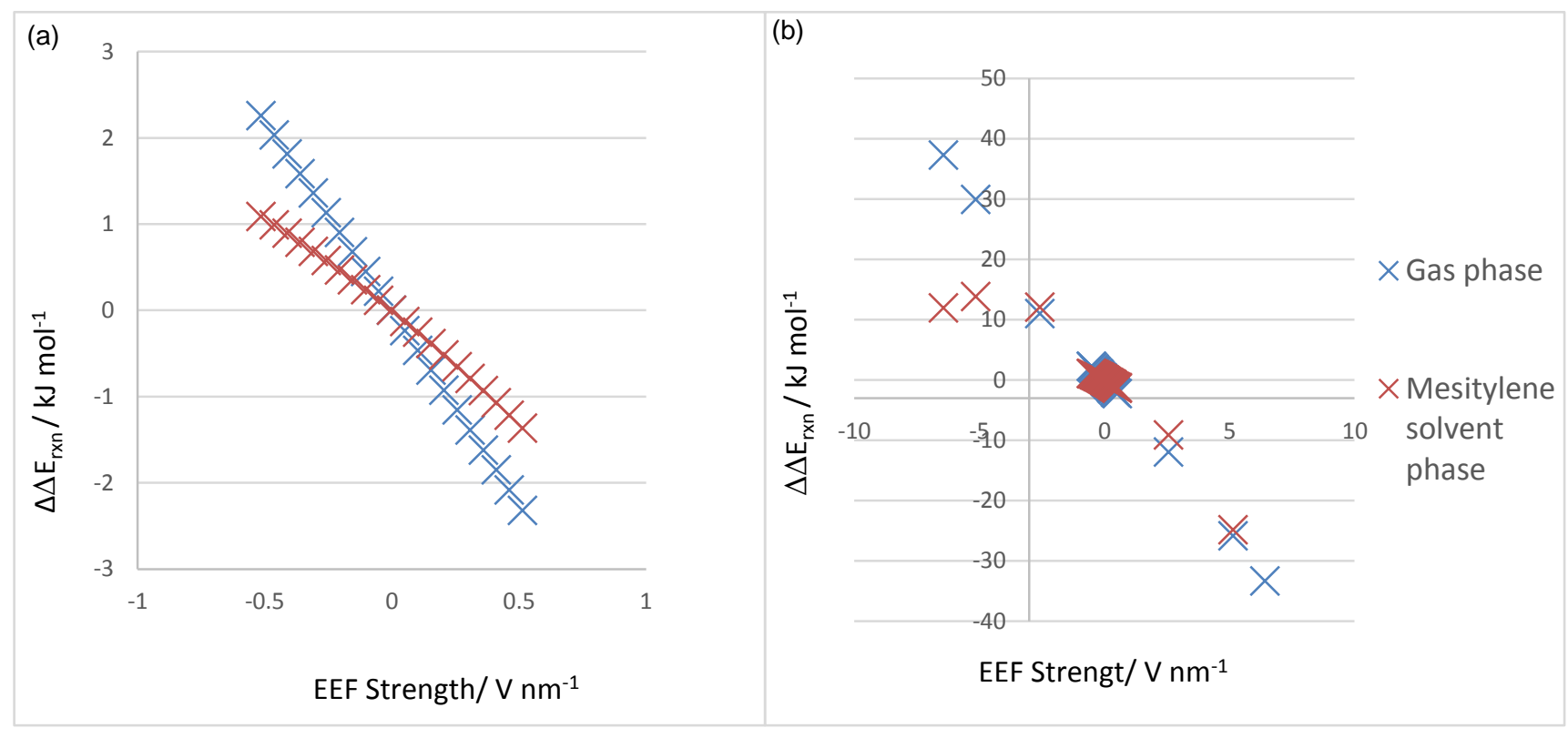

Figure S17. (a) Change in the homolysis reaction energy $\left(\Delta \Delta E \mathrm{~kJ} \mathrm{~mol}^{-1}\right)$ as a function of applied EEF referenced to the same reaction with no $E E F$. 
The results in Figure S17 were calculated for the field aligned consistently along the reaction axis. To assess the effect of field direction, sensitivity analysis was undertaken at a field strength of +0.0010 au. The orientations used were calculated to encompass a spherical shell around the species of interest, and hence were referenced using a spherical coordinate system where the polar angle $(\theta)$ is the angle from the z-axis to the current orientation and the azimuthal angle $(\phi)$ is the angle on the $x-y$ plane of the current orientation. The radial distance $(r)$ is the strength of the applied EEF. Figure S18a below shows the two spherical coordinates and how they are represented in relation to the xyz planes, while Figure $\mathrm{S} 18 \mathrm{~b}-\mathrm{C}$ shows the effect of orientation on the reaction on the reaction energies. It is clear that the results are highly directional, and that greater field effects than those in Figure S17 are indeed possible depending on how the species are actually aligned in the field. At the same time, the tethering of the alkoxyamine to the surface of the STM means there is a limit to the orientations accessible, which is why there is still a qualitative difference in the effects of positive and negative bias, as observed experimentally.

(a)

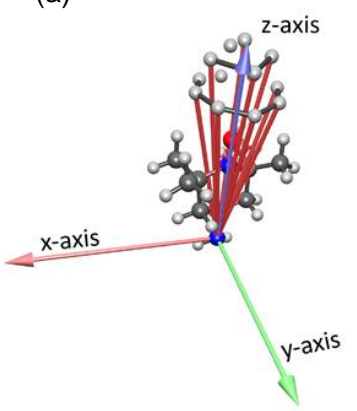

(b)

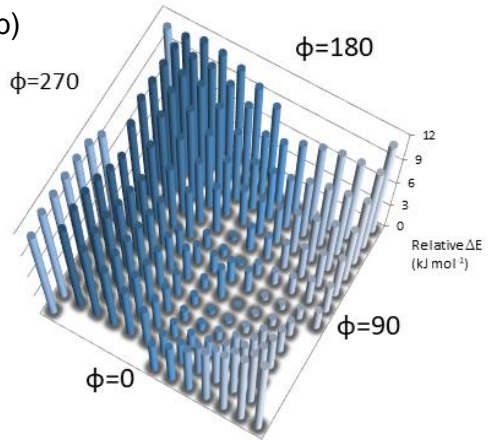

(c)

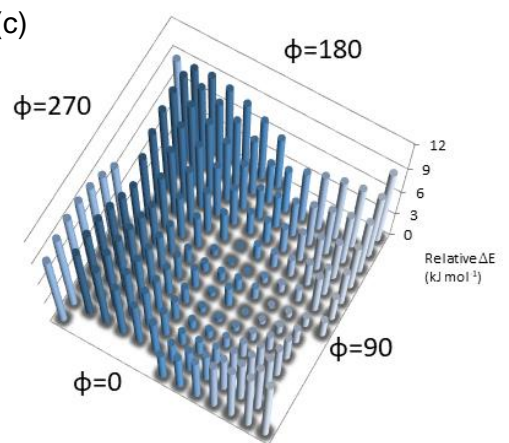

Figure S18. (a) Relation between spherical coordinate and xyz coordinate systems for product TEMPO molecule (b) The effect of an applied EEF of 0.0010 au on the gas phase reaction energy as function of orientation in the field referenced to the lowest reaction energy in the dataset. (c) The same results as (b) but in mesitylene solution. 
Gas phase numbers for Figure S17a including raw energies for each species (below)

\begin{tabular}{|c|c|c|c|c|c|}
\hline $\begin{array}{l}\text { Field strength } \\
\text { (atomic units) }\end{array}$ & $\begin{array}{c}\text { Field } \\
\text { strength } \\
(\mathrm{V} / \mathrm{nm})\end{array}$ & $\begin{array}{l}\text { 4-amino-TEMPO } \\
\text { radical electronic } \\
\text { energy (Hartrees) }\end{array}$ & $\begin{array}{c}\text { 4-ethylaniline } \\
\text { radical } \\
\text { electronic } \\
\text { energy } \\
\text { (Hartrees) }\end{array}$ & $\begin{array}{c}\text { Reactant } \\
\text { alkoxyamine } \\
\text { electronic } \\
\text { energy } \\
\text { (Hartrees) }\end{array}$ & $\begin{array}{c}\text { Reaction } \\
\text { electronic } \\
\text { energy } \\
(\mathrm{kJ} / \mathrm{mol})\end{array}$ \\
\hline-0.0125 & -6.4 & -538.828253 & -365.446635 & -904.356502 & 214.3 \\
\hline-0.01 & -5.1 & -538.827494 & -365.439034 & -904.345330 & 206.9 \\
\hline-0.005 & -2.6 & -538.828510 & -365.428020 & -904.328138 & 188.0 \\
\hline-0.001 & -0.5 & -538.831734 & -365.423505 & -904.323515 & 179.3 \\
\hline-0.0009 & -0.5 & -538.831842 & -365.423439 & -904.323471 & 179.0 \\
\hline-0.0008 & -0.4 & -538.831951 & -365.423375 & -904.323431 & 178.8 \\
\hline-0.0007 & -0.4 & -538.832062 & -365.423313 & -904.323394 & 178.6 \\
\hline-0.0006 & -0.3 & -538.832174 & -365.423253 & -904.323360 & 178.4 \\
\hline-0.0005 & -0.3 & -538.832288 & -365.423195 & -904.323329 & 178.1 \\
\hline-0.0004 & -0.2 & -538.832402 & -365.423139 & -904.323301 & 177.9 \\
\hline-0.0003 & -0.2 & -538.832518 & -365.423086 & -904.323277 & 177.7 \\
\hline-0.0002 & -0.1 & -538.832635 & -365.423034 & -904.323256 & 177.4 \\
\hline-0.0001 & -0.1 & -538.832754 & -365.422984 & -904.323238 & 177.2 \\
\hline 0 & 0.0 & -538.832874 & -365.422933 & -904.323224 & 177.0 \\
\hline 0.0001 & 0.1 & -538.832996 & -365.422891 & -904.323212 & 176.8 \\
\hline 0.0002 & 0.1 & -538.833118 & -365.422848 & -904.323204 & 176.5 \\
\hline 0.0003 & 0.2 & -538.833242 & -365.422806 & -904.323199 & 176.3 \\
\hline 0.0004 & 0.2 & -538.833368 & -365.422767 & -904.323197 & 176.1 \\
\hline 0.0005 & 0.3 & -538.833494 & -365.422730 & -904.323199 & 175.8 \\
\hline 0.0006 & 0.3 & -538.833622 & -365.422695 & -904.323203 & 175.6 \\
\hline 0.0007 & 0.4 & -538.833751 & -365.422661 & -904.323211 & 175.4 \\
\hline 0.0008 & 0.4 & -538.833882 & -365.422630 & -904.323222 & 175.1 \\
\hline 0.0009 & 0.5 & -538.834013 & -365.422601 & -904.323236 & 174.9 \\
\hline 0.001 & 0.5 & -538.834148 & -365.422573 & -904.323253 & 174.7 \\
\hline 0.005 & 2.6 & -538.840574 & -365.423016 & -904.326444 & 165.0 \\
\hline 0.01 & 5.1 & -538.851615 & -365.427924 & -904.337108 & 151.1 \\
\hline 0.0125 & 6.4 & -538.858394 & -365.432131 & -904.345243 & 143.7 \\
\hline
\end{tabular}

Mesitylene solvent phase numbers for Figure 17a including raw energies for each species (below)

\begin{tabular}{|c|c|c|c|c|c|}
\hline $\begin{array}{c}\text { Field strength } \\
\text { (atomic units) }\end{array}$ & $\begin{array}{c}\text { Field } \\
\text { strength } \\
\mathbf{( V / n m )}\end{array}$ & $\begin{array}{c}\text { 4-amino-TEMPO } \\
\text { radical electronic } \\
\text { energy (Hartrees) }\end{array}$ & $\begin{array}{c}\text { 4-ethylaniline } \\
\text { radical electronic } \\
\text { energy } \\
\text { (Hartrees) }\end{array}$ & $\begin{array}{c}\text { Reactant } \\
\text { alkoxyamine } \\
\text { electronic energy } \\
\text { (Hartrees) }\end{array}$ & $\begin{array}{c}\text { Reaction } \\
\text { electronic } \\
\text { energy } \\
\text { (kJ/mol) }\end{array}$ \\
\hline-0.0125 & -6.4 & -538.841890 & -365.463810 & -904.374534 & 180.7 \\
\hline-0.01 & -5.1 & -538.840090 & -365.454122 & -904.363778 & 182.6 \\
\hline-0.005 & -2.6 & -538.839571 & -365.440038 & -904.348487 & 180.8 \\
\hline-0.001 & -0.5 & -538.842081 & -365.434374 & -904.341162 & 169.9 \\
\hline-0.0009 & -0.5 & -538.842177 & -365.434294 & -904.341141 & 169.8 \\
\hline-0.0008 & -0.4 & -538.842274 & -365.434216 & -904.341123 & 169.7 \\
\hline
\end{tabular}




\begin{tabular}{|c|c|c|c|c|c|}
\hline-0.0007 & -0.4 & -538.842373 & -365.434141 & -904.341108 & 169.6 \\
\hline-0.0006 & -0.3 & -538.842474 & -365.434069 & -904.341096 & 169.5 \\
\hline-0.0005 & -0.3 & -538.842576 & -365.433999 & -904.341088 & 169.4 \\
\hline-0.0004 & -0.2 & -538.842680 & -365.433932 & -904.341082 & 169.3 \\
\hline-0.0003 & -0.2 & -538.842786 & -365.433867 & -904.341080 & 169.2 \\
\hline-0.0002 & -0.1 & -538.842893 & -365.433806 & -904.341081 & 169.0 \\
\hline-0.0001 & -0.1 & -538.843002 & -365.433746 & -904.341085 & 168.9 \\
\hline 0 & 0.0 & -538.843111 & -365.433688 & -904.341093 & 168.8 \\
\hline 0.0001 & 0.1 & -538.843224 & -365.433635 & -904.341103 & 168.7 \\
\hline 0.0002 & 0.1 & -538.843338 & -365.433584 & -904.341117 & 168.5 \\
\hline 0.0003 & 0.2 & -538.843453 & -365.433535 & -904.341133 & 168.4 \\
\hline 0.0004 & 0.2 & -538.843570 & -365.433488 & -904.341153 & 168.3 \\
\hline 0.0005 & 0.3 & -538.843688 & -365.433444 & -904.341176 & 168.1 \\
\hline 0.0006 & 0.3 & -538.843808 & -365.433402 & -904.341202 & 168.0 \\
\hline 0.0007 & 0.4 & -538.843930 & -365.433363 & -904.341232 & 167.9 \\
\hline 0.0008 & 0.4 & -538.844054 & -365.433327 & -904.341264 & 167.7 \\
\hline 0.0009 & 0.5 & -538.844179 & -365.433293 & -904.341300 & 167.6 \\
\hline 0.001 & 0.5 & -538.844305 & -365.433261 & -904.341339 & 167.4 \\
\hline 0.005 & 2.6 & -538.850702 & -365.433899 & -904.345412 & 159.7 \\
\hline 0.01 & 5.1 & -538.862366 & -365.439983 & -904.357179 & 144.0 \\
\hline 0.0125 & 6.4 & -538.869745 & -365.445154 & -904.365786 & 133.6 \\
\hline
\end{tabular}

Gas phase numbers for Figure S18b including raw energies for each species (below)

\begin{tabular}{|c|c|c|c|c|c|}
\hline Polar angle & $\begin{array}{c}\text { Azimuthal } \\
\text { angle }\end{array}$ & $\begin{array}{c}\text { 4-amino-TEMPO } \\
\text { radical electronic } \\
\text { energy (Hartrees) }\end{array}$ & $\begin{array}{c}\text { 4-ethylaniline } \\
\text { radical } \\
\text { electronic } \\
\text { energy } \\
\text { (Hartrees) }\end{array}$ & $\begin{array}{c}\text { Reactant } \\
\text { alkoxyamine } \\
\text { electronic } \\
\text { energy } \\
\text { (Hartrees) }\end{array}$ & $\begin{array}{c}\text { Relative } \\
\text { reaction } \\
\text { electronic } \\
\text { energy } \\
\text { (kJ/mol) }\end{array}$ \\
\hline 0 & 0 & -538.834148 & -365.423501 & -904.323253 & -4.8 \\
\hline 15 & 0 & -538.834073 & -365.423478 & -904.323000 & -5.2 \\
\hline 15 & 90 & -538.834286 & -365.423592 & -904.323154 & -5.6 \\
\hline 15 & 180 & -538.834141 & -365.423487 & -904.323510 & -4.0 \\
\hline 15 & 270 & -538.833927 & -365.423370 & -904.323356 & -3.6 \\
\hline 30 & 0 & -538.833923 & -365.423418 & -904.322767 & -5.2 \\
\hline 30 & 45 & -538.834186 & -365.423571 & -904.322773 & -6.3 \\
\hline 30 & 90 & -538.834332 & -365.423638 & -904.323067 & -6.1 \\
\hline 30 & 135 & -538.834278 & -365.423584 & -904.323475 & -4.7 \\
\hline 30 & 180 & -538.834053 & -365.423436 & -904.323757 & -3.0 \\
\hline 30 & 225 & -538.833787 & -365.423279 & -904.323749 & -1.9 \\
\hline 30 & 270 & -538.833638 & -365.423209 & -904.323457 & -2.1 \\
\hline 30 & 315 & -538.833695 & -365.423267 & -904.323051 & -3.5 \\
\hline 45 & 0 & -538.833707 & -365.423328 & -904.322569 & -4.9 \\
\hline 45 & 30 & -538.833963 & -365.423480 & -904.322523 & -6.1 \\
\hline 45 & 60 & -538.834174 & -365.423593 & -904.322680 & -6.6 \\
\hline
\end{tabular}




\begin{tabular}{|c|c|c|c|c|c|}
\hline 45 & 90 & -538.834284 & -365.423639 & -904.322998 & -6.1 \\
\hline 45 & 120 & -538.834266 & -365.423607 & -904.323389 & -5.0 \\
\hline 45 & 150 & -538.834123 & -365.423504 & -904.323748 & -3.4 \\
\hline 45 & 180 & -538.833891 & -365.423354 & -904.323979 & -1.8 \\
\hline 45 & 210 & -538.833631 & -365.423198 & -904.324020 & -0.6 \\
\hline 45 & 240 & -538.833415 & -365.423078 & -904.323864 & -0.1 \\
\hline 45 & 270 & -538.833302 & -365.423029 & -904.323551 & -0.5 \\
\hline 45 & 300 & -538.833323 & -365.423065 & -904.323164 & -1.7 \\
\hline 45 & 330 & -538.833472 & -365.423175 & -904.322804 & -3.3 \\
\hline 60 & 0 & -538.833438 & -365.423215 & -904.322420 & -4.3 \\
\hline 60 & 22 & -538.833676 & -365.423359 & -904.322354 & -5.5 \\
\hline 60 & 45 & -538.833893 & -365.423481 & -904.322433 & -6.2 \\
\hline 60 & 67 & -538.834057 & -365.423564 & -904.322644 & -6.3 \\
\hline 60 & 90 & -538.834145 & -365.423599 & -904.322954 & -5.8 \\
\hline 60 & 112 & -538.834143 & -365.423578 & -904.323315 & -4.8 \\
\hline 60 & 135 & -538.834053 & -365.423507 & -904.323672 & -3.4 \\
\hline 60 & 157 & -538.833885 & -365.423392 & -904.323970 & -1.9 \\
\hline 60 & 180 & -538.833664 & -365.423251 & -904.324163 & -0.4 \\
\hline 60 & 202 & -538.833424 & -365.423103 & -904.324224 & 0.7 \\
\hline 60 & 225 & -538.833201 & -365.422973 & -904.324144 & 1.5 \\
\hline 60 & 247 & -538.833030 & -365.422882 & -904.323936 & 1.6 \\
\hline 60 & 270 & -538.832940 & -365.422846 & -904.323632 & 1.1 \\
\hline 60 & 292 & -538.832945 & -365.422869 & -904.323276 & 0.1 \\
\hline 60 & 315 & -538.833042 & -365.422949 & -904.322920 & -1.3 \\
\hline 60 & 337 & -538.833215 & -365.423071 & -904.322619 & -2.8 \\
\hline 75 & 0 & -538.833136 & -365.423091 & -904.322333 & -3.4 \\
\hline 75 & 18 & -538.833348 & -365.423221 & -904.322262 & -4.5 \\
\hline 75 & 36 & -538.833551 & -365.423338 & -904.322296 & -5.3 \\
\hline 75 & 54 & -538.833724 & -365.423432 & -904.322430 & -5.6 \\
\hline 75 & 72 & -538.833852 & -365.423495 & -904.322653 & -5.5 \\
\hline 75 & 90 & -538.833923 & -365.423521 & -904.322940 & -5.0 \\
\hline 75 & 108 & -538.833930 & -365.423509 & -904.323264 & -4.2 \\
\hline 75 & 126 & -538.833873 & -365.423458 & -904.323593 & -3.0 \\
\hline 75 & 144 & -538.833755 & -365.423374 & -904.323893 & -1.7 \\
\hline 75 & 162 & -538.833589 & -365.423262 & -904.324136 & -0.3 \\
\hline 75 & 180 & -538.833388 & -365.423133 & -904.324299 & 0.9 \\
\hline 75 & 198 & -538.833173 & -365.423000 & -904.324365 & 2.0 \\
\hline 75 & 216 & -538.832965 & -365.422876 & -904.324330 & 2.8 \\
\hline 75 & 234 & -538.832785 & -365.422773 & -904.324196 & 3.2 \\
\hline 75 & 252 & -538.832652 & -365.422704 & -904.323978 & 3.2 \\
\hline 75 & 270 & -538.832579 & -365.422676 & -904.323697 & 2.7 \\
\hline 75 & 288 & -538.832574 & -365.422691 & -904.323378 & 1.8 \\
\hline 75 & 306 & -538.832637 & -365.422749 & -904.323052 & 0.7 \\
\hline
\end{tabular}




\begin{tabular}{|c|c|c|c|c|c|}
\hline 75 & 324 & -538.832762 & -365.422842 & -904.322750 & -0.7 \\
\hline 75 & 342 & -538.832933 & -365.422960 & -904.322502 & -2.1 \\
\hline 90 & 0 & -538.832820 & -365.422965 & -904.322318 & -2.3 \\
\hline 90 & 15 & -538.833003 & -365.423079 & -904.322250 & -3.3 \\
\hline 90 & 30 & -538.833182 & -365.423185 & -904.322257 & -4.0 \\
\hline 90 & 45 & -538.833344 & -365.423276 & -904.322340 & -4.5 \\
\hline 90 & 60 & -538.833479 & -365.423347 & -904.322492 & -4.6 \\
\hline 90 & 75 & -538.833578 & -365.423394 & -904.322703 & -4.4 \\
\hline 90 & 90 & -538.833635 & -365.423415 & -904.322958 & -4.0 \\
\hline 90 & 105 & -538.833646 & -365.423407 & -904.323240 & -3.2 \\
\hline 90 & 120 & -538.833610 & -365.423372 & -904.323528 & -2.3 \\
\hline 90 & 135 & -538.833530 & -365.423310 & -904.323803 & -1.2 \\
\hline 90 & 150 & -538.833409 & -365.423227 & -904.324047 & 0.0 \\
\hline 90 & 165 & -538.833256 & -365.423125 & -904.324242 & 1.2 \\
\hline 90 & 180 & -538.833081 & -365.423013 & -904.324376 & 2.3 \\
\hline 90 & 195 & -538.832895 & -365.422896 & -904.324440 & 3.2 \\
\hline 90 & 210 & -538.832712 & -365.422785 & -904.324431 & 4.0 \\
\hline 90 & 225 & -538.832544 & -365.422686 & -904.324348 & 4.5 \\
\hline 90 & 240 & -538.832403 & -365.422607 & -904.324198 & 4.7 \\
\hline 90 & 255 & -538.832300 & -365.422555 & -904.323991 & 4.5 \\
\hline 90 & 270 & -538.832242 & -365.422533 & -904.323742 & 4.1 \\
\hline 90 & 285 & -538.832233 & -365.422543 & -904.323465 & 3.3 \\
\hline 90 & 300 & -538.832273 & -365.422584 & -904.323179 & 2.4 \\
\hline 90 & 315 & -538.832360 & -365.422653 & -904.322904 & 1.2 \\
\hline 90 & 330 & -538.832486 & -365.422745 & -904.322658 & 0.0 \\
\hline 90 & 345 & -538.832643 & -365.422851 & -904.322457 & -1.2 \\
\hline 105 & 0 & -538.832511 & -365.422847 & -904.322378 & -1.0 \\
\hline 105 & 18 & -538.832724 & -365.422979 & -904.322308 & -2.1 \\
\hline 105 & 36 & -538.832927 & -365.423099 & -904.322345 & -2.9 \\
\hline 105 & 54 & -538.833101 & -365.423194 & -904.322485 & -3.2 \\
\hline 105 & 72 & -538.833229 & -365.423259 & -904.322713 & -3.1 \\
\hline 105 & 90 & -538.833300 & -365.423286 & -904.323008 & -2.6 \\
\hline 105 & 108 & -538.833307 & -365.423274 & -904.323339 & -1.7 \\
\hline 105 & 126 & -538.833250 & -365.423224 & -904.323673 & -0.6 \\
\hline 105 & 144 & -538.833132 & -365.423139 & -904.323979 & 0.8 \\
\hline 105 & 162 & -538.832965 & -365.423027 & -904.324225 & 2.1 \\
\hline 105 & 180 & -538.832764 & -365.422896 & -904.324388 & 3.4 \\
\hline 105 & 198 & -538.832548 & -365.422761 & -904.324454 & 4.5 \\
\hline 105 & 216 & -538.832340 & -365.422634 & -904.324415 & 5.3 \\
\hline 105 & 234 & -538.832159 & -365.422530 & -904.324277 & 5.7 \\
\hline 105 & 252 & -538.832026 & -365.422458 & -904.324053 & 5.7 \\
\hline 105 & 270 & -538.831953 & -365.422429 & -904.323764 & 5.2 \\
\hline 105 & 288 & -538.831948 & -365.422444 & -904.323438 & 4.3 \\
\hline
\end{tabular}




\begin{tabular}{|c|c|c|c|c|c|}
\hline 105 & 306 & -538.832012 & -365.422502 & -904.323105 & 3.1 \\
\hline 105 & 324 & -538.832136 & -365.422595 & -904.322798 & 1.7 \\
\hline 105 & 342 & -538.832308 & -365.422715 & -904.322547 & 0.3 \\
\hline 120 & 0 & -538.832231 & -365.422745 & -904.322510 & 0.3 \\
\hline 120 & 22 & -538.832470 & -365.422893 & -904.322449 & -0.9 \\
\hline 120 & 45 & -538.832687 & -365.423019 & -904.322536 & -1.5 \\
\hline 120 & 67 & -538.832852 & -365.423106 & -904.322760 & -1.6 \\
\hline 120 & 90 & -538.832940 & -365.423143 & -904.323085 & -1.1 \\
\hline 120 & 112 & -538.832939 & -365.423125 & -904.323461 & 0.0 \\
\hline 120 & 135 & -538.832848 & -365.423053 & -904.323830 & 1.3 \\
\hline 120 & 157 & -538.832680 & -365.422936 & -904.324135 & 2.9 \\
\hline 120 & 180 & -538.832458 & -365.422792 & -904.324332 & 4.4 \\
\hline 120 & 202 & -538.832217 & -365.422640 & -904.324390 & 5.6 \\
\hline 120 & 225 & -538.831993 & -365.422505 & -904.324302 & 6.3 \\
\hline 120 & 247 & -538.831822 & -365.422410 & -904.324082 & 6.4 \\
\hline 120 & 270 & -538.831732 & -365.422370 & -904.323762 & 5.9 \\
\hline 120 & 292 & -538.831736 & -365.422392 & -904.323391 & 4.8 \\
\hline 120 & 315 & -538.831833 & -365.422473 & -904.323022 & 3.4 \\
\hline 120 & 337 & -538.832008 & -365.422597 & -904.322712 & 1.8 \\
\hline 135 & 0 & -538.832000 & -365.422663 & -904.322708 & 1.6 \\
\hline 135 & 30 & -538.832258 & -365.422822 & -904.322669 & 0.5 \\
\hline 135 & 60 & -538.832469 & -365.422942 & -904.322843 & 0.0 \\
\hline 135 & 90 & -538.832580 & -365.422993 & -904.323183 & 0.5 \\
\hline 135 & 120 & -538.832562 & -365.422962 & -904.323596 & 1.7 \\
\hline 135 & 150 & -538.832418 & -365.422858 & -904.323971 & 3.4 \\
\hline 135 & 180 & -538.832185 & -365.422703 & -904.324208 & 5.0 \\
\hline 135 & 210 & -538.831924 & -365.422540 & -904.324243 & 6.2 \\
\hline 135 & 240 & -538.831707 & -365.422412 & -904.324071 & 6.7 \\
\hline 135 & 270 & -538.831593 & -365.422358 & -904.323735 & 6.2 \\
\hline 135 & 300 & -538.831614 & -365.422393 & -904.323325 & 5.0 \\
\hline 135 & 330 & -538.831764 & -365.422506 & -904.322949 & 3.3 \\
\hline 150 & 0 & -538.831833 & -365.422606 & -904.322954 & 2.9 \\
\hline 150 & 45 & -538.832098 & -365.422767 & -904.322972 & 1.8 \\
\hline 150 & 90 & -538.832245 & -365.422842 & -904.323294 & 2.1 \\
\hline 150 & 135 & -538.832190 & -365.422788 & -904.323729 & 3.5 \\
\hline 150 & 180 & -538.831964 & -365.422635 & -904.324022 & 5.3 \\
\hline 150 & 225 & -538.831696 & -365.422470 & -904.324002 & 6.4 \\
\hline 150 & 270 & -538.831546 & -365.422391 & -904.323683 & 6.1 \\
\hline 150 & 315 & -538.831604 & -365.422449 & -904.323250 & 4.7 \\
\hline 165 & 0 & -538.831742 & -365.422575 & -904.323231 & 3.9 \\
\hline 165 & 90 & -538.831956 & -365.422698 & -904.323408 & 3.5 \\
\hline 165 & 180 & -538.831810 & -365.422590 & -904.323785 & 5.2 \\
\hline 165 & 270 & -538.831594 & -365.422465 & -904.323609 & 5.6 \\
\hline
\end{tabular}




\begin{tabular}{|l|l|l|l|l|l|}
\hline 180 & 0 & -538.831734 & -365.422570 & -904.323515 & 4.7 \\
\hline
\end{tabular}

Mesitylene solvent phase numbers for Figure S18c including raw energies for each species (below)

\begin{tabular}{|c|c|c|c|c|c|}
\hline Polar angle & $\begin{array}{l}\text { Azimuthal } \\
\text { angle }\end{array}$ & $\begin{array}{l}\text { 4-amino-TEMPO } \\
\text { radical electronic } \\
\text { energy (Hartrees) }\end{array}$ & $\begin{array}{l}\text { 4-ethylaniline } \\
\text { radical } \\
\text { electronic } \\
\text { energy } \\
\text { (Hartrees) }\end{array}$ & $\begin{array}{l}\text { Reactant } \\
\text { alkoxyamine } \\
\text { electronic } \\
\text { energy } \\
\text { (Hartrees) }\end{array}$ & $\begin{array}{l}\text { Relative } \\
\text { reaction } \\
\text { electronic } \\
\text { energy } \\
(\mathrm{kJ} / \mathrm{mol})\end{array}$ \\
\hline 0 & 0 & -538.844305 & -365.434373 & -904.341339 & -4.3 \\
\hline 15 & 0 & -538.844405 & -365.434362 & -904.341375 & -4.4 \\
\hline 15 & 90 & -538.844305 & -365.434471 & -904.341235 & -4.8 \\
\hline 15 & 180 & -538.844129 & -365.434337 & -904.341294 & -3.8 \\
\hline 15 & 270 & -538.844231 & -365.434226 & -904.341438 & -3.4 \\
\hline 30 & 0 & -538.844421 & -365.434307 & -904.341399 & -4.3 \\
\hline 30 & 45 & -538.844396 & -365.434465 & -904.341245 & -5.0 \\
\hline 30 & 90 & -538.844231 & -365.434515 & -904.341132 & -5.0 \\
\hline 30 & 135 & -538.844020 & -365.434430 & -904.341129 & -4.2 \\
\hline 30 & 180 & -538.843890 & -365.434257 & -904.341247 & -3.1 \\
\hline 30 & 225 & -538.843920 & -365.434094 & -904.341417 & -2.3 \\
\hline 30 & 270 & -538.844087 & -365.434041 & -904.341528 & -2.3 \\
\hline 30 & 315 & -538.844294 & -365.434130 & -904.341515 & -3.2 \\
\hline 45 & 0 & -538.844354 & -365.434214 & -904.341408 & -3.8 \\
\hline 45 & 30 & -538.844357 & -365.434376 & -904.341263 & -4.6 \\
\hline 45 & 60 & -538.844260 & -365.434482 & -904.341128 & -5.0 \\
\hline 45 & 90 & -538.844087 & -365.434507 & -904.341034 & -4.9 \\
\hline 45 & 120 & -538.843883 & -365.434446 & -904.341010 & -4.2 \\
\hline 45 & 150 & -538.843705 & -365.434313 & -904.341070 & -3.3 \\
\hline 45 & 180 & -538.843603 & -365.434141 & -904.341203 & -2.2 \\
\hline 45 & 210 & -538.843606 & -365.433975 & -904.341370 & -1.3 \\
\hline 45 & 240 & -538.843709 & -365.433860 & -904.341518 & -0.9 \\
\hline 45 & 270 & -538.843884 & -365.433832 & -904.341604 & -1.1 \\
\hline 45 & 300 & -538.844082 & -365.433897 & -904.341606 & -1.8 \\
\hline 45 & 330 & -538.844253 & -365.434038 & -904.341532 & -2.8 \\
\hline 60 & 0 & -538.844207 & -365.434093 & -904.341402 & -3.1 \\
\hline 60 & 22 & -538.844222 & -365.434247 & -904.341268 & -3.9 \\
\hline 60 & 45 & -538.844167 & -365.434368 & -904.341138 & -4.4 \\
\hline 60 & 67 & -538.844048 & -365.434438 & -904.341026 & -4.6 \\
\hline 60 & 90 & -538.843882 & -365.434451 & -904.340947 & -4.4 \\
\hline 60 & 112 & -538.843694 & -365.434404 & -904.340916 & -3.9 \\
\hline 60 & 135 & -538.843514 & -365.434303 & -904.340944 & -3.1 \\
\hline 60 & 157 & -538.843371 & -365.434162 & -904.341030 & -2.1 \\
\hline 60 & 180 & -538.843288 & -365.434001 & -904.341164 & -1.1 \\
\hline
\end{tabular}




\begin{tabular}{|c|c|c|c|c|c|}
\hline 60 & 202 & -538.843279 & -365.433842 & -904.341322 & -0.2 \\
\hline 60 & 225 & -538.843342 & -365.433712 & -904.341475 & 0.3 \\
\hline 60 & 247 & -538.843467 & -365.433633 & -904.341595 & 0.5 \\
\hline 60 & 270 & -538.843633 & -365.433617 & -904.341661 & 0.3 \\
\hline 60 & 292 & -538.843816 & -365.433668 & -904.341667 & -0.3 \\
\hline 60 & 315 & -538.843988 & -365.433778 & -904.341617 & -1.2 \\
\hline 60 & 337 & -538.844125 & -365.433928 & -904.341523 & -2.2 \\
\hline 75 & 0 & -538.843991 & -365.433955 & -904.341382 & -2.2 \\
\hline 75 & 18 & -538.844011 & -365.434096 & -904.341262 & -3.0 \\
\hline 75 & 36 & -538.843981 & -365.434215 & -904.341143 & -3.5 \\
\hline 75 & 54 & -538.843902 & -365.434302 & -904.341033 & -3.8 \\
\hline 75 & 72 & -538.843781 & -365.434349 & -904.340941 & -3.9 \\
\hline 75 & 90 & -538.843630 & -365.434354 & -904.340876 & -3.7 \\
\hline 75 & 108 & -538.843462 & -365.434317 & -904.340845 & -3.2 \\
\hline 75 & 126 & -538.843296 & -365.434239 & -904.340856 & -2.6 \\
\hline 75 & 144 & -538.843148 & -365.434129 & -904.340911 & -1.7 \\
\hline 75 & 162 & -538.843035 & -365.433994 & -904.341007 & -0.8 \\
\hline 75 & 180 & -538.842967 & -365.433848 & -904.341135 & 0.1 \\
\hline 75 & 198 & -538.842952 & -365.433704 & -904.341280 & 0.9 \\
\hline 75 & 216 & -538.842990 & -365.433576 & -904.341424 & 1.5 \\
\hline 75 & 234 & -538.843076 & -365.433479 & -904.341550 & 1.8 \\
\hline 75 & 252 & -538.843201 & -365.433424 & -904.341644 & 1.9 \\
\hline 75 & 270 & -538.843353 & -365.433416 & -904.341695 & 1.7 \\
\hline 75 & 288 & -538.843515 & -365.433457 & -904.341701 & 1.1 \\
\hline 75 & 306 & -538.843674 & -365.433543 & -904.341665 & 0.4 \\
\hline 75 & 324 & -538.843814 & -365.433664 & -904.341593 & -0.5 \\
\hline 75 & 342 & -538.843923 & -365.433806 & -904.341496 & -1.4 \\
\hline 90 & 0 & -538.843720 & -365.433812 & -904.341353 & -1.2 \\
\hline 90 & 15 & -538.843741 & -365.433935 & -904.341246 & -1.9 \\
\hline 90 & 30 & -538.843726 & -365.434044 & -904.341141 & -2.4 \\
\hline 90 & 45 & -538.843675 & -365.434132 & -904.341042 & -2.8 \\
\hline 90 & 60 & -538.843592 & -365.434193 & -904.340953 & -3.0 \\
\hline 90 & 75 & -538.843480 & -365.434225 & -904.340880 & -2.9 \\
\hline 90 & 90 & -538.843347 & -365.434226 & -904.340827 & -2.7 \\
\hline 90 & 105 & -538.843204 & -365.434196 & -904.340801 & -2.3 \\
\hline 90 & 120 & -538.843058 & -365.434137 & -904.340803 & -1.8 \\
\hline 90 & 135 & -538.842922 & -365.434051 & -904.340838 & -1.1 \\
\hline 90 & 150 & -538.842805 & -365.433945 & -904.340887 & -0.4 \\
\hline 90 & 165 & -538.842716 & -365.433824 & -904.340984 & 0.4 \\
\hline 90 & 180 & -538.842662 & -365.433696 & -904.341100 & 1.2 \\
\hline 90 & 195 & -538.842645 & -365.433570 & -904.341226 & 1.9 \\
\hline 90 & 210 & -538.842666 & -365.433454 & -904.341373 & 2.5 \\
\hline 90 & 225 & -538.842724 & -365.433357 & -904.341492 & 2.9 \\
\hline
\end{tabular}




\begin{tabular}{|c|c|c|c|c|c|}
\hline 90 & 240 & -538.842814 & -365.433287 & -904.341591 & 3.1 \\
\hline 90 & 255 & -538.842929 & -365.433249 & -904.341662 & 3.1 \\
\hline 90 & 270 & -538.843061 & -365.433246 & -904.341700 & 2.9 \\
\hline 90 & 285 & -538.843201 & -365.433279 & -904.341705 & 2.4 \\
\hline 90 & 300 & -538.843340 & -365.433345 & -904.341678 & 1.8 \\
\hline 90 & 315 & -538.843469 & -365.433439 & -904.341624 & 1.1 \\
\hline 90 & 330 & -538.843579 & -365.433555 & -904.341547 & 0.3 \\
\hline 90 & 345 & -538.843665 & -365.433682 & -904.341455 & -0.5 \\
\hline 105 & 0 & -538.843414 & -365.433673 & -904.341317 & -0.2 \\
\hline 105 & 18 & -538.843434 & -365.433815 & -904.341190 & -0.9 \\
\hline 105 & 36 & -538.843404 & -365.433936 & -904.341067 & -1.5 \\
\hline 105 & 54 & -538.843325 & -365.434023 & -904.340957 & -1.8 \\
\hline 105 & 72 & -538.843205 & -365.434071 & -904.340867 & -1.8 \\
\hline 105 & 90 & -538.843054 & -365.434075 & -904.340806 & -1.6 \\
\hline 105 & 108 & -538.842887 & -365.434036 & -904.340782 & -1.1 \\
\hline 105 & 126 & -538.842721 & -365.433956 & -904.340781 & -0.5 \\
\hline 105 & 144 & -538.842573 & -365.433843 & -904.340852 & 0.4 \\
\hline 105 & 162 & -538.842460 & -365.433706 & -904.340961 & 1.3 \\
\hline 105 & 180 & -538.842392 & -365.433557 & -904.341098 & 2.2 \\
\hline 105 & 198 & -538.842377 & -365.433410 & -904.341247 & 3.1 \\
\hline 105 & 216 & -538.842415 & -365.433281 & -904.341390 & 3.7 \\
\hline 105 & 234 & -538.842501 & -365.433183 & -904.341536 & 4.1 \\
\hline 105 & 252 & -538.842626 & -365.433127 & -904.341628 & 4.2 \\
\hline 105 & 270 & -538.842777 & -365.433120 & -904.341675 & 3.9 \\
\hline 105 & 288 & -538.842939 & -365.433163 & -904.341674 & 3.4 \\
\hline 105 & 306 & -538.843098 & -365.433252 & -904.341628 & 2.6 \\
\hline 105 & 324 & -538.843238 & -365.433375 & -904.341547 & 1.7 \\
\hline 105 & 342 & -538.843347 & -365.433521 & -904.341440 & 0.7 \\
\hline 120 & 0 & -538.843093 & -365.433546 & -904.341280 & 0.9 \\
\hline 120 & 22 & -538.843108 & -365.433705 & -904.341133 & 0.1 \\
\hline 120 & 45 & -538.843053 & -365.433828 & -904.340997 & -0.5 \\
\hline 120 & 67 & -538.842934 & -365.433899 & -904.340887 & -0.6 \\
\hline 120 & 90 & -538.842769 & -365.433909 & -904.340817 & -0.4 \\
\hline 120 & 112 & -538.842582 & -365.433858 & -904.340781 & 0.1 \\
\hline 120 & 135 & -538.842402 & -365.433753 & -904.340836 & 1.0 \\
\hline 120 & 157 & -538.842260 & -365.433606 & -904.340948 & 2.1 \\
\hline 120 & 180 & -538.842178 & -365.433438 & -904.341102 & 3.1 \\
\hline 120 & 202 & -538.842168 & -365.433275 & -904.341271 & 4.0 \\
\hline 120 & 225 & -538.842231 & -365.433142 & -904.341424 & 4.6 \\
\hline 120 & 247 & -538.842355 & -365.433061 & -904.341559 & 4.9 \\
\hline 120 & 270 & -538.842521 & -365.433047 & -904.341617 & 4.6 \\
\hline 120 & 292 & -538.842703 & -365.433103 & -904.341607 & 4.0 \\
\hline 120 & 315 & -538.842875 & -365.433219 & -904.341536 & 3.0 \\
\hline
\end{tabular}




\begin{tabular}{|c|c|c|c|c|c|}
\hline 120 & 337 & -538.843011 & -365.433375 & -904.341421 & 1.9 \\
\hline 135 & 0 & -538.842779 & -365.433438 & -904.341245 & 1.9 \\
\hline 135 & 30 & -538.842782 & -365.433606 & -904.341081 & 1.0 \\
\hline 135 & 60 & -538.842685 & -365.433714 & -904.340942 & 0.7 \\
\hline 135 & 90 & -538.842513 & -365.433737 & -904.340861 & 0.8 \\
\hline 135 & 120 & -538.842310 & -365.433670 & -904.340848 & 1.5 \\
\hline 135 & 150 & -538.842133 & -365.433528 & -904.340947 & 2.6 \\
\hline 135 & 180 & -538.842032 & -365.433347 & -904.341111 & 3.8 \\
\hline 135 & 210 & -538.842034 & -365.433174 & -904.341294 & 4.7 \\
\hline 135 & 240 & -538.842137 & -365.433056 & -904.341438 & 5.1 \\
\hline 135 & 270 & -538.842311 & -365.433029 & -904.341529 & 5.0 \\
\hline 135 & 300 & -538.842508 & -365.433101 & -904.341506 & 4.2 \\
\hline 135 & 330 & -538.842679 & -365.433252 & -904.341400 & 3.1 \\
\hline 150 & 0 & -538.842493 & -365.433352 & -904.341213 & 2.8 \\
\hline 150 & 45 & -538.842469 & -365.433518 & -904.341038 & 2.0 \\
\hline 150 & 90 & -538.842304 & -365.433566 & -904.340919 & 2.0 \\
\hline 150 & 135 & -538.842094 & -365.433472 & -904.340960 & 2.9 \\
\hline 150 & 180 & -538.841965 & -365.433287 & -904.341122 & 4.1 \\
\hline 150 & 225 & -538.841994 & -365.433116 & -904.341307 & 5.0 \\
\hline 150 & 270 & -538.842161 & -365.433063 & -904.341418 & 5.0 \\
\hline 150 & 315 & -538.842366 & -365.433162 & -904.341370 & 4.1 \\
\hline 165 & 0 & -538.842255 & -365.433292 & -904.341185 & 3.5 \\
\hline 165 & 90 & -538.842156 & -365.433404 & -904.341025 & 3.1 \\
\hline 165 & 180 & -538.841982 & -365.433258 & -904.341134 & 4.2 \\
\hline 165 & 270 & -538.842082 & -365.433143 & -904.341275 & 4.6 \\
\hline 180 & 0 & -538.842081 & -365.433260 & -904.341147 & 4.0 \\
\hline
\end{tabular}

\section{Geometries at zero applied EEF}

\section{4-amino-TEMPO radical:}

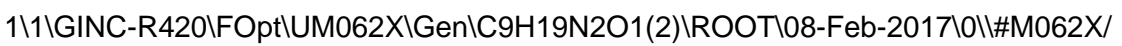
gen 6D SCF=Tight IOP(2/17=4) INT(grid=ultrafine) Opt maxdisk=161061273 $6 \|$ a3\|0,2\N,-0.5933405987,-1.2350755837,-1.589271146।C,-0.5378847461, $0.1397048429,-1.0905492292 \backslash \mathrm{C}, 0.7591290799,0.3332963283,-0.3150062657 \backslash \mathrm{C}$ $, 0.9576554136,1.7579329716,0.2140785883 \backslash \mathrm{N}, 0.6640789806,2.7436234396,-0$ $.8650830021 \backslash \mathrm{C},-0.4149335696,2.5727940953,-1.8794303254 \backslash \mathrm{C},-0.5266895509$ ,1.0965888076,-2.2760466282\C,-1.7364460209,3.1165998393,-1.3129802788 IC, $-0.0188334108,3.3985835902,-3.1053134227 \backslash \mathrm{C}, 0.0603870463,2.049844914$ $4,1.4276158452 \backslash C, 2.4214389769,1.9499151512,0.6166950727 \backslash 0,1.0185465715$ ,3.9452340303,-0.6297855233\H,-1.4517677833,-1.3888087512,-2.114976829 $7 \backslash \mathrm{H},-0.6001490736,-1.8951583537,-0.8139410905 \backslash \mathrm{H},-1.3935946692,0.377377$ $6115,-0.4369655074 \backslash \mathrm{H}, 1.587016731,0.0681181933,-0.9858658659 \backslash \mathrm{H}, 0.804190$ $2826,-0.3548806322,0.5401498719 \backslash \mathrm{H}, 0.3217448287,0.8191813605,-2.9155635$ $461 \backslash \mathrm{H},-1.4374069174,0.975848429,-2.8784844791 \backslash \mathrm{H},-2.1387701411,2.490603$ $4614,-0.5119559255 \backslash \mathrm{H},-2.4847512111,3.1658479078,-2.111499405 \mathrm{H},-1.5765$ $738186,4.125462646,-0.9211635096 \backslash \mathrm{H}, 0.9741079832,3.1043355409,-3.459012$ $2561 \backslash \mathrm{H}, 0.0003360277,4.4636618407,-2.8668439975 \backslash \mathrm{H},-0.7437210127,3.22344$ $11768,-3.9073042144 \backslash \mathrm{H}, 0.3969524514,1.4550634934,2.2837570701 \backslash \mathrm{H}, 0.13402$ $85004,3.1098852472,1.6879546861 \mathrm{H},-0.9894611509,1.8082371726,1.2410096$ 
01\H,3.0728963196,1.858400138,-0.2578594453\Н,2.6991354983,1.179594370 5,1.3438919716\H,2.5761729834,2.9345097206,1.0619581865।IVersion=ES64L -G09RevE.01 \State $=2-A \backslash H F=-538.8328837 \backslash S 2=0.754872 \backslash S 2-1=0 . \mid S 2 A=0.750014$ $\backslash$ RMSD $=6.164 \mathrm{e}-09 \backslash \mathrm{RMSF}=6.852 \mathrm{e}-06 \backslash \mathrm{Dipole}=-0.9247804,-1.0294309,0.2051583 \backslash$ Quadrupole=1.9252178,-3.9095131,1.9842953,0.2857103,1.8108139,-3.46479 87\PG=C01 [X(C9H19N2O1)]॥@

\section{4-ethylaniline radical:}

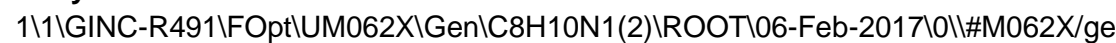
n 6D SCF=Tight IOP(2/17=4) INT(grid=ultrafine) Opt maxdisk=1073741824\ la2 b4 c4|\0,2\C,-1.8800688989,0.876281094,-0.0531493274\C,-1.81224460 $16,2.2558276199,-0.1394201848 \mid C,-0.5766420811,2.9214965892,-0.10495921$ $55 \backslash C, 0.5951159626,2.150916447,0.0121758539 \backslash C, 0.5281665322,0.7752461696$ ,0.0984573703\C,-0.7108684318,0.079053373,0.0700301426\C,-0.7567979167 $,-1.3310113108,0.1621913329 \backslash \mathrm{C},-2.0260227283,-2.1194959313,0.136496515 \backslash$ $\mathrm{N},-0.5057301301,4.3075669457,-0.2508751192 \backslash \mathrm{H},-2.8539177045,0.395436267$ $1,-0.0804789051 \backslash \mathrm{H},-2.726445564,2.8369060039,-0.2401246267 \backslash \mathrm{H}, 1.56141119$ $89,2.6502113308,0.0294080027 \backslash \mathrm{H}, 1.4471727072,0.2009786396,0.1910605851 \backslash$ $\mathrm{H}, 0.1853152208,-1.8637979886,0.2541988459 \mathrm{H},-2.5889337011,-1.958094898$ $1,-0.7942006165 \backslash \mathrm{H},-2.6975080549,-1.8410574085,0.9615709129 \backslash \mathrm{H},-1.826966$ $1128,-3.1902928609,0.220212047 \backslash \mathrm{H}, 0.3270010301,4.7376965908,0.132008421$ $1 \backslash \mathrm{H},-1.341614726,4.8109883274,0.0191359658||$ Version=ES64L-G09RevE.01\S tate $=2-A \backslash H F=-365.4229344 \backslash S 2=0.777507 \backslash S 2-1=0 . \mid S 2 A=0.750415 \backslash R M S D=7.085 e-$ 09\RMSF $=3.867 \mathrm{e}-06 \backslash$ Dipole $=-0.0371226,0.5107886,0.3860503 \backslash$ Quadrupole $=1.7$ $743384,5.388216,-7.1625544,0.2994722,0.8047516,2.5754325 \backslash P G=C 01[X(C 8 H$ 10N1)]1@

\section{Reactant alkoxyamine:}

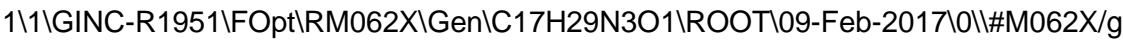
en 6 D SCF=Tight IOP(2/17=4) INT(grid=ultrafine) Opt maxdisk=2684354560

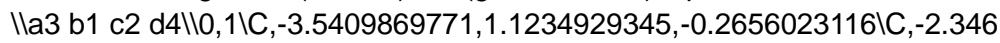
$6001873,1.3072391031,-0.9545603701 \backslash \mathrm{C},-3.5625077365,1.1095698286,1.1347$ 74274\C,-2.3556104051,1.2858649251,1.8226402803\C,-1.1666884864,1.4652 $856759,1.1228454416 \backslash \mathrm{C},-1.1401071873,1.4784167774,-0.2724092302 \backslash \mathrm{N},-4.77$ $07427914,0.9833729347,1.8304706929 \backslash \mathrm{C}, 0.1574765733,1.7184468587,-1.0172$ $609934 \backslash \mathrm{C}, 0.3697299552,3.2135075925,-1.2496292242 \backslash \mathrm{N}, 1.2555438528,0.2250$ $872163,-2.5269400897 \backslash \mathrm{C}, 1.7342281723,0.5830472718,-3.8915411795 \backslash \mathrm{C}, 0.912$ $8240404,-1.2026716729,-2.2632093714 \backslash \mathrm{C}, 2.1473492803,-2.0396948892,-2.63$ $22965017 \backslash \mathrm{C}, 2.669351054,-1.7972841768,-4.0479853164 \backslash \mathrm{C}, 2.9427031556,-0.3$ $085750917,-4.209359294 \backslash \mathrm{C}, 2.219448111,2.0344175058,-3.8403546049 \backslash \mathrm{C}, 0.66$ $86089446,0.487655875,-5.0014987016 \mid C,-0.3490899947,-1.7112434748,-2.98$ $37737976 \backslash \mathrm{C}, 0.6783971844,-1.358575635,-0.7552754179 \mathrm{~W}, 3.8811382215,-2.5$ $464791059,-4.3834313465 \backslash \mathrm{H},-4.4702363888,0.9932481524,-0.8157874708 \backslash \mathrm{H},-$ $2.3461584618,1.3038526656,-2.0414205321 \backslash \mathrm{H},-2.3515229919,1.2789873813,2$ $.9102253406 \backslash \mathrm{H},-0.2379677114,1.5868443365,1.678327063 \backslash \mathrm{H},-5.5018617781,0$ $.5037277447,1.3196819334 \mathrm{H},-4.6798301231,0.6133804974,2.7687076828 \backslash \mathrm{H}, 0$ $.9956965859,1.313752351,-0.4404832385 \backslash \mathrm{H}, 0.2616287442,3.7548410858,-0.3$ $042884957 \backslash \mathrm{H},-0.3879766063,3.589117765,-1.9462715626 \backslash \mathrm{H}, 1.3624625001,3.4$ 179645523,-1.6562531388\Н,1.8989482879,-3.1007838694,-2.4923522075\H,2 $.9564752581,-1.7996695538,-1.9259347998 \backslash \mathrm{H}, 1.9096292142,-2.1056071394,-$ $4.7773342041 \backslash \mathrm{H}, 3.2777660146,-0.1028337197,-5.2325213194 \backslash \mathrm{H}, 3.7667125643$ ,-0.0333421614,-3.5331409084\Н,2.8760279604,2.1855957873,-2.9772007523 $\backslash \mathrm{H}, 1.3762576017,2.7267473664,-3.7749437567 \backslash \mathrm{H}, 2.7806522091,2.265921192$, $-4.7522170747 \mathrm{H}, 0.4908804984,-0.5323832927,-5.3462251056 \backslash \mathrm{H}, 1.002925743$ $6,1.0685540811,-5.868177062 \backslash \mathrm{H},-0.278165544,0.910068068,-4.653313267 \backslash \mathrm{H}$, $-0.682877142,-2.6409984437,-2.5097998763 \backslash \mathrm{H},-1.1539520918,-0.9762487359$ ,-2.8900299083\H,-0.1897192894,-1.9233407449,-4.042281268\H,1.50883902 $03,-0.9246954228,-0.1882662355 \backslash \mathrm{H}, 0.6122155454,-2.4239041779,-0.5079549$ $029 \mathrm{H},-0.2559775938,-0.8844070785,-0.4414097607 \backslash \mathrm{H}, 4.6234494704,-2.3167$ $795927,-3.7229835403 \backslash \mathrm{H}, 3.7146708106,-3.5474143199,-4.2978972941 \backslash 0,0.10$ 25649134,1.0172727727,-2.2683952757 $\mid$ Version=ES64L-G09RevE.01\State=1- 
$\mathrm{A} \backslash \mathrm{HF}=-904.3232232 \backslash \mathrm{RMSD}=3.070 \mathrm{e}-09 \backslash \mathrm{RMSF}=5.198 \mathrm{e}-06 \backslash \mathrm{Dipole}=-0.3873336,-0.6$ 910446,0.7743493\Quadrupole $=2.7907164,-2.3784723,-0.4122442,5.1590341$, 1.3181168,-4.463837\PG=C01 [X(C17H29N3O1)]॥@ 


\section{S8. Theoretical study of the electrochemical cleavage of the alkoxyamine}

Theoretical Methodology. All geometry optimizations were performed using the M06-2X functional, ${ }^{19}$ in combination with a $6-31+G(d, p)$ basis set. ${ }^{20}$ Entropies, thermal corrections and zero-point vibrational energies were scaled by recommend scale factors. ${ }^{21}$ All geometries were verified as local minima (possessing no imaginary frequencies). Improved single-point energies were calculated using the high-level composite ab initio G3(MP2,CC)(+) method, a variation of standard G3(MP2,CC) ${ }^{22}$ where calculations with the $6-31 \mathrm{G}(\mathrm{d})$ basis set are replaced with corresponding $6-31+G(d)$. These high-level calculations where utilized in conjunction with the ONIOM approximation ${ }^{23}$ for larger systems, with either standard G3(MP2,CC) or UMP2 used to model remote substituents effects. The SMD solvent model ${ }^{24}$ was used to relax gas-phase structures to acetonitrile solution-phase (at the UM06-2X/6-31+G(d,p) level of theory). Free energies of solvation were then calculated using the COSMO-RS model. ${ }^{25}$ The ADF package ${ }^{26}$ was used to computed COSMO-RS solvation free energies on solution-phase geometries at the BP/TZP level of theory (as it was parameterized for), and the remaining parameters were kept as default values. ${ }^{27}$ The methodology used in this work has been extensively benchmarked, including for oxidation potentials and ion-pairing energies. ${ }^{28}$ All standard ab initio molecular orbital theory, density functional theory (DFT) calculations were carried out using Gaussian $09^{15}$ and Molpro $2015^{29}$ software packages. Field calculations were performed using M06-2X/6-31+G(d,p) with a $0.5 \mathrm{~V} / \mathrm{nm}$ field orientated anti-parallel with the dipole moment of the respective species. For field calculations, the SMD solvent model $^{24}$ (instead of COSMO-RS) was employed, using acetonitrile and DCM as solvents. The effect of an electric field on the Gibbs free-energy of fragmentation was estimated from the respective changes in the M06-2X/6-31+G(d,p) electronic energies in the presence of a SMD solvent field. Calculations with point charges were performed using $M 06-2 X / 6-31+G(d, p)$ with QChem $4.4^{30}$, with the point charge positioned where the centre of the perchlorate anion was in the corresponding $\mathrm{ClO}_{4}$ ion-pair. Consistent with previous work, ${ }^{31}$ we have adopted the electron convention based on Fermi-Dirac statistics (EC-FD), as recommended by Bartmess. ${ }^{32}$ We used the experimentally determined absolute potential of the $\mathrm{Fc}^{+} / \mathrm{Fc}$ couple (in acetonitrile) of $4.980 \mathrm{~V}$, taken by correcting a recent value for the Saturated Calomel Electrode (SCE) in acetonitrile ${ }^{33}$ with the reduction potential of $\mathrm{Fc}^{+} / \mathrm{Fc}$ in acetonitrile relative to the SCE. ${ }^{34}$ This value was found to be nearly identical to a corresponding computationally derived value (of $4.988 \mathrm{~V}$ ), calculated using similar high-level theory in conjunction with COSMO-RS. ${ }^{31}$ To determine the oxidation potential of the title alkoxyamine in DCM, we used the experimentally determined absolute potential of the $\mathrm{Fc}^{+} / \mathrm{Fc}$ couple (in $\mathrm{DCM}$ ) of $5.010 \mathrm{~V} .{ }^{35}$ As before, this value was found to be very similar to a corresponding computationally derived value (of $4.927 \mathrm{~V}$ ) calculated using similar high-level theory in conjunction with COSMO-RS. ${ }^{31}$ 


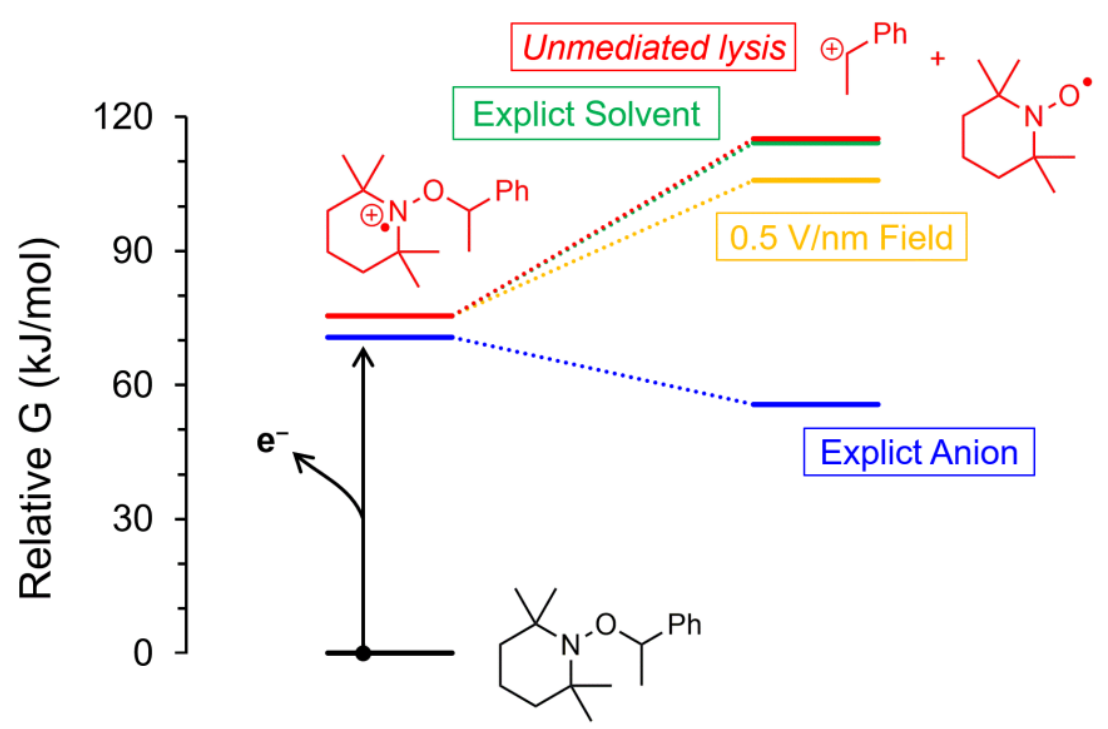

Figure S19. Electrochemical cleavage of alkoxyamines. Theoretical potential energy surface for the oxidative cleavage of alkoxyamine 3 in DCM. The figure shows the effect of electrostatic environment (either solvent or electrolyte anions) on the reaction energ ies of alkoxyamine cation radicals decomposition. The homolysis of the unperturbed ('free') radical-cation (red pathway) is strongly thermodynamically disfavoured. However, homolysis can is made more favourable by a static electric field, and by interactions with an explicit anion. Unlike acetonitrile, an explicit solvent molecule does not aid homolysis. 


\section{Optimized geometries and total energies}<smiles>C[N+](C)=O</smiles>

Oxoammonium CORE<smiles>CN(C)[O]</smiles>

TEMPO CORE

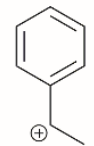

Ethylbenzene cat

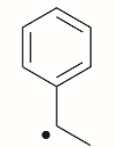

Ethylbenzene rad<smiles>CC(ON(C)C)c1ccccc1</smiles>

Alkoxyam CORE<smiles>CC(ON(C)C)c1ccccc1</smiles>

Alkoxyam radcat CORE<smiles>CC(C)OC(=O)N(C)OC(C)c1ccccc1</smiles>

Alkoxyam radcat $\mathrm{ClO}_{4} \mathrm{CORE}$

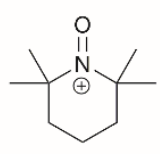

Oxoammonium

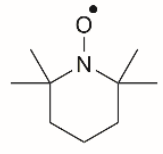

TEMPO

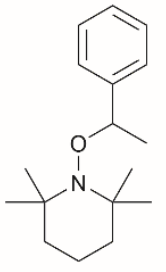

Alkoxyam<smiles>COC(C)c1ccccc1</smiles>

Ethylbenzene cat $\mathrm{ClO}_{4}$
$\mathrm{ClO}_{4}^{\ominus}$

Perchorate<smiles>C1CCCCC1</smiles>

$\mathrm{ACN}$

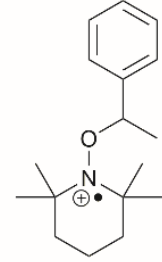

Alkoxyam radcat<smiles>CC(ON1C(C)(C)CCCC1(C)C)c1ccccc1</smiles>

Alkoxyam radcat $\mathrm{ClO}_{4}$

Scheme S4. Chemical structures of all the species in this study.

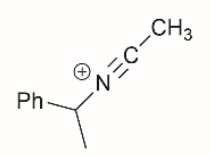

Ethylbenzene cat $\mathrm{ACN}$

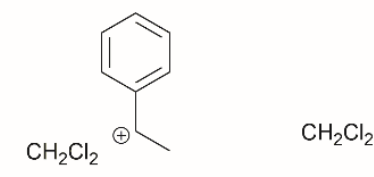

Ethylbenzene cat DCM

DCM 
Contributions of raw energies, thermal corrections, entropies and solvation corrections to the total Gibbs free-energies and enthalpies. Note that the standard state gas to solution-phase correction has been included in the solvation energies $\left(G_{\text {solv }}\right)$. Unless otherwise specified, units are given in hartrees.

\begin{tabular}{|c|c|c|c|c|c|c|c|c|c|}
\hline \multirow[b]{2}{*}{ Species } & \multicolumn{3}{|c|}{ Thermal Corrections $\left(25^{\circ} \mathrm{C}\right)$} & \multicolumn{6}{|c|}{ Raw Energies } \\
\hline & $\begin{array}{c}\mathrm{S} \\
(\mathrm{J} / \mathrm{mol} \\
\mathrm{K})\end{array}$ & $\begin{array}{l}\text { Thermal } \\
\text { correction }\end{array}$ & ZPVE & $\begin{array}{l}\text { UM06-2X/ } \\
6-31+G(d, p)\end{array}$ & $\begin{array}{c}\text { UMP2/ } \\
6-31+G(d)\end{array}$ & $\begin{array}{l}\text { UMP2/ } \\
\text { 6-31G(d) }\end{array}$ & $\begin{array}{c}\text { UMP2/ } \\
\text { GTMP2Large }\end{array}$ & $\begin{array}{c}\text { URCCSD(T)/ } \\
6-31 G+(d)\end{array}$ & $\begin{array}{l}\operatorname{URCCSD}(T) / \\
6-31 G(d)\end{array}$ \\
\hline Alkoxyam CORE & 461.80 & 0.013264 & 0.228060 & -519.805238 & -518.322512 & -518.288460 & -518.894409 & -518.477201 & -518.441968 \\
\hline Alkoxyam radcat CORE & 476.99 & 0.013847 & 0.227885 & -519.531017 & -518.051200 & -518.025621 & -518.614797 & -518.211577 & -518.184608 \\
\hline TEMPO CORE & 303.33 & 0.006243 & 0.082102 & -209.628346 & -209.058357 & -209.041799 & -209.298081 & -209.118784 & -209.101151 \\
\hline Oxoammonium CORE & 292.45 & 0.006161 & 0.082909 & -209.345062 & -208.795703 & -208.788501 & -209.031103 & -208.851027 & -208.843077 \\
\hline TEMPO & 435.45 & 0.012924 & 0.255725 & -483.523430 & -482.091358 & -482.064110 & -482.662112 & -482.254390 & -482.224544 \\
\hline Oxoammonium & 432.16 & 0.012952 & 0.257021 & -483.266593 & -481.853581 & -481.834276 & -482.419218 & -482.011711 & -481.990085 \\
\hline
\end{tabular}


Ethylbenzene rad

Ethylbenzene cat

Alkoxyam

Alkoxyam radcat
357.43

351.09

0.008393

0.008314

583.46

0.020185

0.020895
0.139109

0.141033

0.401430

0.401444
$-310.097869$

$-309.848900$

$-793.689654$

$-793.422158$ $\begin{array}{lll}-309.138650 & -309.119494 & -309.467993\end{array}$

$-308.937340 \quad-308.926884 \quad-309.258863$

$-791.346817 \quad-791.296751 \quad-792.249822$

$\begin{array}{lll}-791.083085 & -791.040910 & -791.975910\end{array}$
$-309.277550$

$-309.040819$

$-$
$-309.259634$

$-309.030290$

$-791.551217$

$-791.303507$ 
Table S1 Oxidation Potentials (continued)

\begin{tabular}{|c|c|c|c|c|c|c|c|c|}
\hline \multirow[b]{2}{*}{ Species } & \multicolumn{3}{|c|}{ Raw Energies } & \multicolumn{5}{|c|}{ Thermochemistry $\left(25^{\circ} \mathrm{C}\right)$} \\
\hline & G3(MP2,CC)(+) & G3(MP2,CC) & ONIOM & $\begin{array}{c}\text { ONIOM G } \\
\text { (gas) }\end{array}$ & $\begin{array}{c}\Delta \mathbf{G} \text { solv } \\
(\mathrm{ACN})\end{array}$ & $\begin{array}{l}\text { ONIOM G } \\
\text { (ACN) }\end{array}$ & $\begin{array}{c}\Delta \mathbf{G} \text { solv } \\
\text { (DCM) }\end{array}$ & $\begin{array}{l}\text { ONIOM G } \\
\text { (DCM) }\end{array}$ \\
\hline Alkoxyam CORE & -519.359727 & -519.358546 & - & - & - & - & - & - \\
\hline Alkoxyam radcat CORE & -519.080359 & -519.078969 & - & - & - & - & - & - \\
\hline TEMPO CORE & -209.475433 & -209.474359 & - & - & - & - & - & - \\
\hline Oxoammonium CORE & -209.199383 & -209.198635 & - & - & - & - & - & - \\
\hline TEMPO & -483.130329 & -483.127731 & -483.128805 & -482.909605 & -0.006821 & -482.916426 & -0.009518 & -482.919123 \\
\hline Oxoammonium & -482.878564 & -482.876242 & -482.876990 & -482.656093 & -0.068703 & -482.724796 & -0.073447 & -482.729540 \\
\hline Ethylbenzene rad & -309.799122 & -309.800362 & -309.799122 & -309.692210 & -0.003375 & -309.695584 & -0.004626 & -309.696836 \\
\hline Ethylbenzene cat & -309.550602 & -309.550529 & -309.550602 & -309.441125 & -0.072621 & -309.513746 & -0.074808 & -309.515933 \\
\hline Alkoxyam & - & -793.003177 & -793.004359 & -792.649001 & -0.009203 & -792.658204 & -0.012491 & -792.661492 \\
\hline Alkoxyam radcat & - & -792.731952 & -792.733341 & -792.379979 & -0.063475 & -792.443454 & -0.070208 & -792.450187 \\
\hline
\end{tabular}


Table S2 lon-pairing Energies

\begin{tabular}{|c|c|c|c|c|c|c|c|}
\hline \multirow[b]{2}{*}{ Species } & \multicolumn{3}{|c|}{ Thermal Corrections $\left(25^{\circ} \mathrm{C}\right)$} & \multicolumn{4}{|c|}{ Raw Energies } \\
\hline & $\begin{array}{c}\mathrm{S} \\
(\mathrm{J} / \mathrm{mol} \mathrm{K})\end{array}$ & $\begin{array}{l}\text { Thermal } \\
\text { correction }\end{array}$ & ZPVE & $\begin{array}{c}\text { UM06-2X/ } \\
6-31+G(d, p)\end{array}$ & $\begin{array}{c}\text { UMP2/ } \\
6-31+G(d)\end{array}$ & $\begin{array}{c}\text { UMP2/ } \\
\text { GTMP2Large }\end{array}$ & $\begin{array}{c}\text { URCCSD(T)/ } \\
6-31+G(d)\end{array}$ \\
\hline Ethylbenzene cat $\mathrm{ClO}_{4}$ & 460.77 & 0.012775 & 0.160736 & -1070.715152 & -1068.643159 & -1069.450990 & -1068.771629 \\
\hline Perchlorate & 265.83 & 0.005029 & 0.014974 & -760.674608 & -759.511145 & -759.991598 & -759.541332 \\
\hline Ethylbenzene cat ACN & 445.90 & 0.012182 & 0.190130 & -442.593518 & -441.325996 & -441.787467 & -441.458568 \\
\hline Ethylbenzene cat DCM & 505.03 & 0.014023 & 0.170641 & -1269.473894 & -1267.334294 & -1267.891967 & -1267.483959 \\
\hline DCM & 269.92 & 0.004506 & 0.028880 & -959.611725 & -958.381301 & -958.616964 & -958.428452 \\
\hline Alkoxyam radcat CORE & 476.99 & 0.013847 & 0.227885 & -519.531017 & -518.051200 & -518.614797 & -518.211577 \\
\hline Alkoxyam radcat $\mathrm{ClO}_{4} \mathrm{CORE}$ & 589.99 & 0.019026 & 0.245405 & -1280.348125 & -1277.702386 & -1278.749611 & -1277.894041 \\
\hline Alkoxyam radcat & 607.41 & 0.020895 & 0.401444 & -793.422158 & -791.083085 & -791.975910 & - \\
\hline
\end{tabular}


Table S2 lon-pairing Energies (continued)

\begin{tabular}{|c|c|c|c|c|c|c|c|}
\hline \multirow[b]{2}{*}{ Species } & \multicolumn{2}{|c|}{ Raw Energies } & \multicolumn{5}{|c|}{ Thermochemistry $\left(25^{\circ} \mathrm{C}\right)$} \\
\hline & G3(MP2,CC)(+) & ONIOM & $\begin{array}{c}\text { ONIOM G } \\
\text { (gas) }\end{array}$ & $\begin{array}{c}\Delta \mathbf{G} \text { solv } \\
\text { (ACN) }\end{array}$ & $\begin{array}{c}\text { ONIOM G } \\
\text { (ACN) }\end{array}$ & $\begin{array}{c}\Delta \mathbf{G} \text { solv } \\
(\mathrm{DCM})\end{array}$ & $\begin{array}{c}\text { ONIOM G } \\
\text { (DCM) }\end{array}$ \\
\hline Ethylbenzene cat & -309.550602 & -309.550602 & -309.441125 & -0.072621 & -309.513746 & -0.074808 & -309.515933 \\
\hline Ethylbenzene cat $\mathrm{ClO}_{4}$ & -1069.918328 & -1069.918328 & -1069.797142 & -0.008866 & -1069.806009 & -0.009912 & -1069.807054 \\
\hline Perchlorate & -760.172393 & -760.172393 & -760.182578 & -0.089051 & -760.271629 & -0.085875 & -760.268453 \\
\hline Ethylbenzene cat ACN & -442.183602 & -442.183602 & -442.031927 & -0.075653 & -442.107580 & - & - \\
\hline $\mathrm{ACN}$ & -132.590859 & -132.590859 & -132.569529 & -0.005268 & -132.574797 & - & - \\
\hline Ethylbenzene cat DCM & -1268.324023 & -1268.324023 & -1268.196710 & - & - & -0.076182 & -1268.272892 \\
\hline DCM & -958.758245 & -958.758245 & -958.755511 & - & - & -0.003384 & -958.758895 \\
\hline Alkoxyam radcat CORE & -519.080359 & - & - & - & - & - & - \\
\hline Alkoxyam radcat $\mathrm{ClO}_{4} \mathrm{CORE}$ & -1279.397058 & - & - & - & - & - & - \\
\hline Alkoxyam radcat & - & -792.441472 & -792.088110 & -0.06347513 & -792.151585 & -0.070208 & -792.158318 \\
\hline Alkoxyam radcat $\mathrm{ClO}_{4}$ & - & 1552.757001 & -1552.393054 & -0.03288353 & -1552.42594 & -0.035517 & -1552.428571 \\
\hline
\end{tabular}




\section{Geometries}

\section{Oxoammonium CORE}

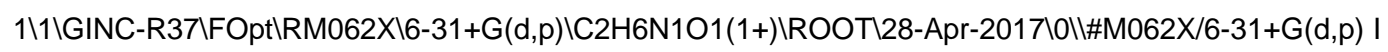
NT(grid=ultrafine) OPT IOP(2/17=4) Freq=noraman maxdisk=134217728॥ITEMPO_cat.CORE.retry1.freq $\backslash 1,1 \backslash \mathrm{C},-0.0023677405,-0.6741254117,1.2748901001 \backslash \mathrm{N}, 0 ., 0.069714493,0 . \backslash \mathrm{C}, 0.0023677405,-0.674125411$ $7,-1.2748901001 \backslash 0,0 ., 1.2500996366,0 . \mid \mathrm{H},-0.7496253911,-1.4672557688,1.198642033 \backslash \mathrm{H},-0.2091591676$ $, 0.0342463009,2.0746318578 \backslash \mathrm{H}, 0.9934536145,-1.1198371852,1.3763002135 \backslash \mathrm{H},-0.9934536145,-1.11983$ 71852,-1.3763002135\H,0.7496253911,-1.4672557688,-1.198642033\Н,0.2091591676,0.0342463009,-2.

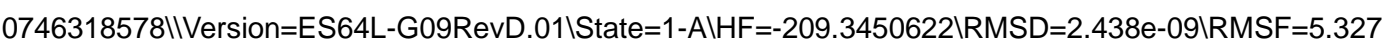
e-05\Dipole=0.,-1.081221,0.।Quadrupole=-1.7901316,-1.4132115,3.2033431,0.,0.1072872,0.।PG=C02 [C 2(N1O1),X(C2H6)]\@

\section{TEMPO CORE}

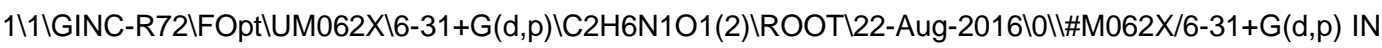
$\mathrm{T}$ (grid=ultrafine) OPT IOP(2/17=4) Freq=noraman maxdisk=134217728 $\mid S G 1$ core.freq $\mid \backslash 0,2 \backslash \mathrm{C},-0.787326$ 043,0.9964996762,-0.1449598012\N,-1.6485224206,-0.1292700368,0.1848046399\C,-1.1538758437,-1. $476535402,-0.0559484155 \backslash \mathrm{O},-2.9085106056,0.0561469974,0.14766795 \backslash \mathrm{H}, 0.2036485799,0.8421576575$ $, 0.2877006824 \backslash \mathrm{H},-1.2442757756,1.8946313429,0.26886783 \backslash \mathrm{H},-0.6956064748,1.1033845049,-1.233894$ 8566ไH,-0.1562283852,-1.5858571904,0.3750916653\H,-1.1084132099,-1.6817364646,-1.1336506348\ $H,-1.8470764146,-2.1723388824,0.4152493588||$ Version=ES64L-G09RevD.01 $\backslash$ State $=2-A^{\prime} \backslash H F=-209.6283$

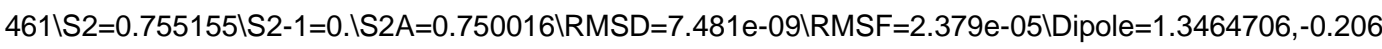
$7637,-0.1998102 \backslash$ Quadrupole=-2.4103254,2.3183453,0.0919801,0.7275052,0.277212,-0.1252644\PG=C S [SG(N1O1),X(C2H6)]॥@

\section{Ethylbenzene cat}

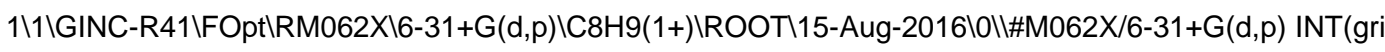
$d=u l t r a f i n e)$ OPT IOP(2/17=4) Freq=noraman maxdisk=671088640 $\backslash S T Y$ cation.C1.freq $\mid \backslash 1,1 \backslash C, 0,0.23951$ 6408,0.4971804244\C,0.,-1.1137073285,0.0319648986\C,0.,-1.3708175072,-1.3195738723\C,0.,-0.2990 $256497,-2.2311860691 \backslash \mathrm{C}, 0 ., 1.0372659123,-1.8053370822 \backslash \mathrm{C}, 0 ., 1.3088832494,-0.4543853531 \backslash \mathrm{H}, 0 .,-1.93$ $45862748,0.7402841278 \backslash \mathrm{H}, 0 .,-2.3908950171,-1.6860868465 \backslash \mathrm{H}, 0 .,-0.5141408425,-3.2960379853 \backslash \mathrm{H}, 0 ., 1$. $8412422481,-2.5321568375 \backslash \mathrm{H}, 0 ., 2.334807419,-0.0963586185 \backslash \mathrm{C}, 0 ., 0.5683702042,1.8461490476 \backslash \mathrm{C}, 0 .,-0$. $3294241419,3.0059917629 \backslash \mathrm{H}, 0 ., 1.6344479642,2.0807942707 \backslash \mathrm{H},-0.8696284776,-0.0842983115,3.63230$ $25756 \backslash \mathrm{H}, 0 .,-1.3914000204,2.7727929811 \backslash \mathrm{H}, 0.8696284776,-0.0842983115,3.6323025756|| V e r s i o n=E S 64$

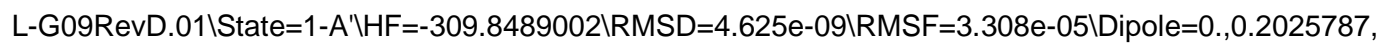
$0.7204674 \backslash$ Quadrupole=-11.715104,-0.7163058,12.4314099,0.,0.,1.0585822\PG=CS [SG(C8H7),X(H2)]\ $@$

\section{Ethylbenzene rad}

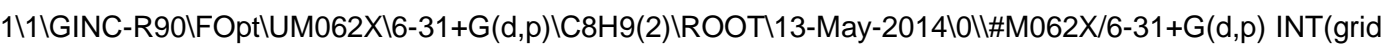
=ultrafine) $\mathrm{OPT} \operatorname{IOP}(2 / 17=4)$ Freq=noraman maxdisk=268435456 $\backslash \mathrm{STY}$ _rad.freq $\backslash 10,2 \backslash \mathrm{C}, 0.0181548384,0$., $0.0256669936 \backslash C, 0.0282393101,0 ., 1.4445119556 \backslash C, 1.2236771545,0 ., 2.1488232662 \backslash C, 2.4475562049,0$. , 1.4740695428\C, $2.461240163,0 ., 0.0744356448 \backslash \mathrm{C}, 1.2734578777,0 .,-0.63719207 \backslash \mathrm{H},-0.9129336693,0 ., 1.9$ 859473132\H,1.2057520322,0.,3.2345953283\Н,3.3789936483,0.,2.0304705812\Н,3.4074862991,0.,-0.4 
$580244897 \backslash \mathrm{H}, 1.2899701618,0,-, 1.7240898238 \backslash \mathrm{C},-1.1889818498,0,-, 0.7205170839 \backslash \mathrm{C},-2.5462303924,0,-$, $0.098280907 \backslash \mathrm{H},-1.1122526783,0 .,-1.8035217255 \backslash \mathrm{H},-3.3285921613,0,-,-0.858865994 \backslash \mathrm{H},-2.698374712,-0$. $8809104244,0.5397122938 \mathrm{H},-2.698374712,0.8809104244,0.5397122938|| V e r s i o n=E S 64 L-G 09 R e v D .01$ IState $=2-A^{\prime \prime}|H F=-310.0978685 \backslash S 2=0.77425 \backslash S 2-1=0| S 2 A=.0.750361 \backslash R M S D=6.578 \mathrm{e}-09 \backslash R M S F=1.151 \mathrm{e}-04 \backslash$ Dipole $=-0.2172301,0 .,-0.0547713$ \Quadrupole $=2.8168637,-4.8748289,2.0579652,0 ., 0.2693204,0 . \mid P G=C$ S [SG(C8H7),X(H2)]॥@

\section{Alkoxyam CORE}

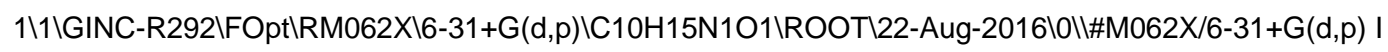
NT(grid=ultrafine) OPT IOP(2/17=4) Freq=noraman maxdisk=134217728|ISG1_PSTY_core.freq $\mid l 0,1 \backslash \mathrm{C},-1$ $.3772967549,1.26444732,-0.8432123204 \mid \mathrm{N},-1.776926877,0.2651236877,0.1374809319 \backslash \mathrm{C},-1.102588869$ ,-1.0052299684,-0.091874199810,-3.1634116372,0.0130144829,-0.0864625929)H,-0.3045164223,1.441 $2034113,-0.7307163228 \mathrm{lH},-1.9121378889,2.1940450654,-0.6426900607 \mathrm{VH},-1.587111815,0.934061699,-$ $1.8724985434 \backslash \mathrm{H},-0.0309938522,-0.8598615554,0.0668027893 \backslash \mathrm{H},-1.2760569375,-1.3853175957,-1.1104$ $199364 \mathrm{H},-1.4686850115,-1.7382203896,0.6301598268 \backslash \mathrm{C},-3.8871935054,0.215932818,1.1234245374 \backslash \mathrm{C}$ ,-5.2728424506,-0.3664260067,0.8812559878\C,-3.9407608135,1.6826769684,1.5026344279lH,-3.3829 $183644,-0.3376233971,1.928033467 \backslash \mathrm{H},-5.7625706023,0.154966309,0.0538624598 \backslash \mathrm{H},-5.8895630505,-0$. 2485604597, 1.77573549161H,-5.1983042447,-1.4275216343,0.6308388344|C,-4.3670280881,2.631765 $9366,0.5679418047 \mathrm{CC},-4.446178342,3.9779394136,0.9128786676 \mathrm{lC},-4.1040360618,4.3913126554,2.20$ 18154928IC,-3.6780675115,3.4519861294,3.1375265636IC,-3.5939013468,2.1044033513,2.784987684 $6 \backslash \mathrm{H},-4.6209551795,2.3077974297,-0.4386711881 \backslash \mathrm{H},-4.7746430728,4.7067798587,0.1780271273 \backslash \mathrm{H},-4.1$ $661347288,5.4409074487,2.4718410086 \backslash \mathrm{H},-3.4025059228,3.7674242795,4.1391852598 \backslash \mathrm{H},-3.24574064$ 9,1.3734427425,3.5107028013||Version=ES64L-G09RevD.011State=1-AlHF=-519.8052385|RMSD=4.61 8e-09IRMSF $=5.691 \mathrm{e}-06 \backslash \mathrm{Dipole}=0.2286759,-0.0275092,0.0713208 \mid$ Quadrupole $=-1.3930255,1.5941619,-$ $0.2011364,-1.430645,-0.5436156,-1.111599 \mid P G=C 01$ [X(C10H15N1O1)]॥@

\section{Alkoxyam radcat CORE}

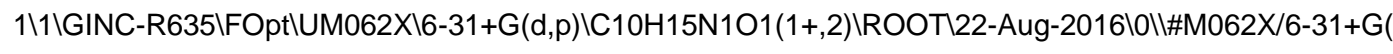
d,p) INT(grid=ultrafine) OPT IOP(2/17=4) Freq=noraman maxdisk=134217728|ISG1_PSTY_core.freq |11,2 IC,-1.5190628536,0.5149125158,-1.8504615044IN,-2.0254393059,0.4272209837,-0.489033831।C,-1.14 $98111134,0.2471436091,0.65925083581 \mathrm{O},-3.3087252636,0.1772254996,-0.4318916554 \mathrm{H},-0.753120845$ $1,1.2915449298,-1.8797259589 \mathrm{H},-2.3501128431,0.764045684,-2.5074810573 \backslash \mathrm{H},-1.0821819921,-0.448$ 4398464,-2.13578460411H,-0.1404649517,0.489300835,0.3311004907\H,-1.1912015229,-0.797188185, $0.9886632585 \backslash \mathrm{H},-1.4544376568,0.9151966578,1.4680651193 \backslash \mathrm{C},-3.9960535796,0.1798507294,0.893163$ 935।C, $-5.4014032628,-0.2739975643,0.553905904 \backslash \mathrm{C},-3.9096365089,1.5520958768,1.509647301 \backslash \mathrm{H},-3.4$ $839926667,-0.5656558725,1.510636172 \backslash \mathrm{H},-5.887552626,0.4482099845,-0.1057961589 \backslash \mathrm{H},-5.973596777$ ,-0.3275342722,1.4826353594\H,-5.3966897164,-1.259135874,0.0832435704\C,-4.1838919216,2.69283 $80544,0.7494123859 \backslash \mathrm{C},-4.1423850698,3.949638699,1.3468905607 \backslash \mathrm{C},-3.8420849622,4.0699223393,2.7$ 043845394IC,-3.5791110239,2.9328779314,3.465621233\C,-3.6045130398, 1.6740926964,2.867072231 4|H,-4.4353747797,2.6013056544,-0.3046084999) $\mathrm{H},-4.3546185188,4.8346450864,0.75652384111 \mathrm{H},-3.8$ $177703056,5.0500576083,3.1685720448 \backslash \mathrm{H},-3.3508993815,3.0235646202,4.5221869124 \backslash \mathrm{H},-3.39893751$ 16,0.7867316194,3.462397575||Version=ES64L-G09RevD.01IState=2-AlHF=-519.5310166IS2 $=0.75474$ 4|S2-1 $=0.1 S 2 A=0.750018|R M S D=5.824 \mathrm{e}-09| R M S F=3.000 \mathrm{e}-06 \mid \mathrm{Dipole}=1.6688609,-1.2404323,-1.146563$ 81Quadrupole $=-1.4218974,-0.7562234,2.1781207,-4.5561589,-6.0862499,4.6837611 \backslash \mathrm{PG}=\mathrm{C} 01[\mathrm{X}(\mathrm{C} 1 \mathrm{OH} 1$ 
5N1O1)]1@

\section{Alkoxyam radcat $\mathrm{ClO}_{4} \mathrm{CORE}$}

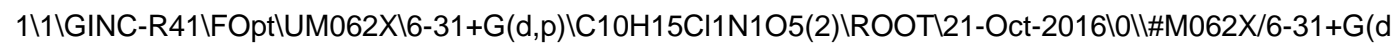
,p) INT(grid=ultrafine) OPT IOP(2/17=4) Freq=noraman maxdisk=536870912।|TEMPO.ClO4.CORE.freq II 0,2\C,0.4796074102,-1.1254162201,-0.9306035448|N,-0.4405787194,-1.0127942017,0.1884023105IC,$0.0154076929,-1.2843156024,1.5530605777 \backslash 0,-1.6977410771,-1.0487691233,-0.1776660039 \backslash 0,1.4921$ $236741,1.1858503198,0.884868107 \backslash \mathrm{Cl}, 0.2973255059,2.0344189816,0.6364548105 \backslash O,-0.2040630585,1$. $7482756919,-0.7313677466 \backslash 0,-0.7656150786,1.6358363092,1.604263870810,0.6228231469,3.4461510$ $672,0.7915675648 \mid \mathrm{C},-2.7582764811,-0.6400240948,0.7956879491 \backslash \mathrm{C},-3.7971597689,0.0242975717,-0.0$ 735279083\C,-3.2147726736,-1.8623833664,1.567424605\H,-2.286639694,0.1184372066, 1.425650376 $9 \mathrm{H},-4.0658674607,-1.5841471443,2.1939753022 \backslash \mathrm{H},-3.5258837753,-2.6607560649,0.8893125421 \backslash \mathrm{H},-2$. $4300350951,-2.2447621236,2.221382725 \backslash \mathrm{C},-5.033590243,-0.5627025668,-0.3474183313 \backslash \mathrm{C},-5.9562097$ 813,0.0989152622,-1.1567438019\C,-5.6421014908,1.3427355149,-1.699606404\C,-4.4045427122,1.92 82086239,-1.4312202546।C,-3.4835743335,1.2745305486,-0.6188657293\H,-5.2943016181,-1.5300066

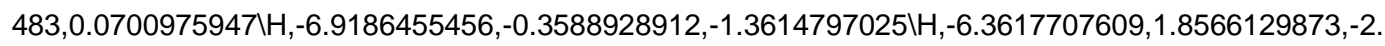
$3292040757 \mathrm{H},-4.1550876391,2.8972150607,-1.8507925472 \backslash \mathrm{H},-2.5133810275,1.7199989716,-0.412654$ $2878 \mathrm{H},-0.0179899643,-0.7216699139,-1.8091635912 \backslash \mathrm{H}, 0.746426994,-2.1799539821,-1.0665746436 \mathrm{H}$, $1.3537578241,-0.5204653986,-0.6829399552 \backslash \mathrm{H}, 1.0336270119,-1.0006076712,1.6075696767 \backslash \mathrm{H},-0.1585$ $637558,-2.3515231517,1.7588643149|\mathrm{H},-0.5702321198,-0.6560839519,2.2488061999| \mid V e r s i o n=E S 64 L-$ G09RevD.01/State $=2-A|H F=-1280.3481247 \backslash \mathrm{S} 2=0.754685 \backslash \mathrm{S} 2-1=0.| \mathrm{S} 2 \mathrm{~A}=0.750018|\mathrm{RMSD}=5.763 \mathrm{e}-09| \mathrm{RM}$ $\mathrm{SF}=1.237 \mathrm{e}-05 \backslash \mathrm{Dipole}=-1.621543,-3.9334921,-0.1664889$ lQuadrupole $=-0.4477074,0.1423181,0.3053893$, -13.1161144,-1.0622209,-7.208872|PG=C01 [X(C10H15Cl1N1O5)]\@

\section{Oxoammonium}

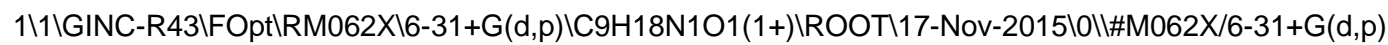
INT(grid=ultrafine) OPT IOP(2/17=4) Freq=noraman maxdisk=268435456||TEMPO_rad.freq\|1,11C,-3.18 $85117461,0.7292222402,-0.1527989793 \mid \mathrm{N},-2.9999212228,-0.6594351796,-0.7661812384 \backslash \mathrm{C},-2.1020686$ 494,-1.7488187313,-0.1768783922lC,-0.8271032731,-1.0803092014,0.3641456646lC,-1.0875509624,0. 1597794628,1.2120239452\C,-1.8291300187,1.2051886194,0.3863541132\C,-3.7140540309,1.6456965 $322,-1.2520691813 \backslash \mathrm{C},-4.2458501434,0.5698932296,0.9580208447 \backslash \mathrm{C},-2.9277249084,-2.4365857545,0.9$ 288065381।C,-1.7920181186,-2.7382272587,-1.2946682797\O,-3.6125549133,-0.9185131268,-1.745685 $0161 \backslash \mathrm{H},-0.1833938838,-0.8172904039,-0.4842065688 \backslash \mathrm{H},-0.2981759552,-1.8494408009,0.9356739856 \backslash$ $\mathrm{H},-0.1318371867,0.5755985433,1.5405998985 \backslash \mathrm{H},-1.6396711617,-0.0911445069,2.1237030953 \backslash \mathrm{H},-1.205$ $6044239,1.5142441307,-0.4615507761 \backslash \mathrm{H},-2.0322989148,2.1058770216,0.9741082698 \backslash \mathrm{H},-3.055201965$ 2,1.6474683884,-2.1242078981\H,-3.7395746241,2.657822803,-0.8417337096) $,-4.7230608955,1.3760$ $617692,-1.5673592303 \mathrm{H},-3.863042209,0.0766933194,1.8500490099 \mathrm{H},-5.1288845526,0.0385399667,0$ $.5931612197 \backslash \mathrm{H},-4.5525254131,1.5812462909,1.2365964646 \backslash \mathrm{H},-3.0246825895,-1.8355002344,1.831468$ $0023 \mid \mathrm{H},-2.3897543035,-3.3517644092,1.1886618248 \backslash \mathrm{H},-3.9194193117,-2.720099146,0.566355202 \backslash \mathrm{H},-1$ $.3505296341,-2.2406762295,-2.1619894524 \backslash \mathrm{H},-2.676239215,-3.2924827693,-1.6127240216 \backslash \mathrm{H},-1.06203$ 55312,-3.4493093558,-0.9010774244||Version=ES64L-G09RevD.01।State=1-A'|HF=-483.2665929|RMS $\mathrm{D}=4.259 \mathrm{e}-09 \backslash \mathrm{RMSF}=1.910 \mathrm{e}-05 \backslash$ Dipole $=0.0860746,0.0344802,0.3352123 \backslash$ Quadrupole $=-0.9631267,4.453$ 0206,-3.4898939,-2.9374227,-0.188231,0.0071713|PG=CS [SG(C1H2N1O1),X(C8H16)]॥@ 


\section{TEMPO}

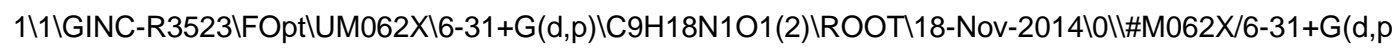
) INT(grid=ultrafine) OPT IOP(2/17=4) Freq=noraman maxdisk=268435456||TEMPO.freq \10,2lC,-3.17956 $95774,0.6943840806,-0.1543978295 \backslash \mathrm{N},-3.0111091496,-0.6645227138,-0.7474070193 \backslash \mathrm{C},-2.1216419643$, $-1.7186166351,-0.1778452386 \mid \mathrm{C},-0.8388983908,-1.0785296864,0.3680998261 \backslash \mathrm{C},-1.098476247,0.15484$ $51927,1.2268757815 \backslash \mathrm{C},-1.835785072,1.1952442897,0.3901943528 \mathrm{IC},-3.6560721161,1.6204220309,-1$. 274930014IC,-4.2509331437,0.638971433,0.9459956346\C,-2.8804531409,-2.4869222357,0.91562096 $16 \mathrm{lC},-1.7714822058,-2.6780921076,-1.3166991768 \backslash \mathrm{O},-3.9914971532,-1.0875822902,-1.4441355913 \mathrm{lH},-$ $0.2044304603,-0.7887816971,-0.4802770318 \mathrm{H},-0.2927318602,-1.8456082837,0.9290419387 \backslash \mathrm{H},-0.1493$ $419719,0.5675071656,1.5834918306 \mathrm{H},-1.6779759482,-0.1079021929,2.1199763312 \backslash \mathrm{H},-1.1988640854$, $1.4793971645,-0.4582368735 \backslash \mathrm{H},-2.0258390315,2.107392652,0.9674537094 \mathrm{H},-2.9604672147,1.586412$ $5622,-2.1183802738 \mid \mathrm{H},-3.7017280578,2.6464577713,-0.8968153984 \mathrm{|H},-4.6440264258,1.3242459484,-1$ $.6301073411 \mathrm{H},-3.9056557026,0.1110272116,1.8382430558 \mathrm{H},-5.1406851559,0.1331938842,0.5613572$ $075 \mathrm{H},-4.5251846193,1.6569024339,1.2404806744 \mathrm{H},-3.0283905726,-1.8899049507,1.8187997648 \mathrm{H},-$ $2.3155619019,-3.3829709303,1.1915076377 \backslash \mathrm{H},-3.858031528,-2.7923787955,0.5329290769 \backslash \mathrm{H},-1.33120$ 05709,-2.1297411036,-2.154490572\H,-2.6612429111,-3.1982355339,-1.6740528207/H, -1.0451436066,3.4128794674,-0.9556946844||Version=ES64L-G09RevD.01/State=2-A'|HF=-483.5234304|S2=0.754861 IS2-1 $=0 .|S 2 A=0.750014 \backslash R M S D=3.991 \mathrm{e}-09 \backslash \mathrm{RMSF}=5.988 \mathrm{e}-06| \mathrm{Dipole}=0.8207978,0.3514986,0.8605761 \backslash \mathrm{Q}$ uadrupole $=-0.7073146,2.3702525,-1.6629378,-1.6385592,-2.2349817,-0.9337956 \mid P G=C S[S G(C 1 H 2 N 1$ O1),X(C8H16)]॥@

\section{Alkoxyam}

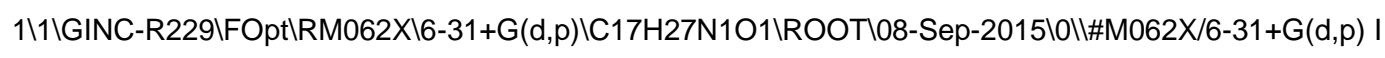
NT(grid=ultrafine) OPT IOP(2/17=4) Freq=noraman maxdisk=671088640॥TEMPO-STY.freq $\mid 0,1 \backslash \mathrm{C},-0.830$ $0608139,-1.3188421974,0.5909753605 \backslash \mathrm{N},-0.8064086134,-0.0580747529,-0.2124239606 / \mathrm{C},-2.11561031$ 32,0.5928874796,-0.5142752389IC,-3.0077842311,-0.457856392,-1.1958366034IC,-3.1474421826,-1.74 68352512,-0.3960306869\C,-1.7589404228,-2.3116879178,-0.1260116051।C,0.5822242865,-1.9174644 $806,0.5709569041 \backslash \mathrm{C},-1.2389011269,-1.1385275734,2.0651154785 \backslash \mathrm{C},-2.8232232315,1.212036822,0.706$ $3657324 \backslash \mathrm{C},-1.8654270392,1.7135872865,-1.5270970176 \backslash \mathrm{O},-0.0074100901,0.8849051027,0.500556730$ $5 \backslash \mathrm{H},-2.5713633158,-0.6917199613,-2.1760443564 \backslash \mathrm{H},-3.9864032471,0.0015863483,-1.3779319753 \backslash \mathrm{H},-3$. $7473327066,-2.4735811426,-0.9539829109 \backslash \mathrm{H},-3.6801338216,-1.5632261205,0.5444902122 \backslash \mathrm{H},-1.29522$ $23765,-2.585119367,-1.0831956919 \mid \mathrm{H},-1.812009323,-3.2230195142,0.481521298 \mathrm{H}, 0.9513452818,-2.0$ $015573461,-0.4554937153 \backslash \mathrm{H}, 0.555160592,-2.9192152984,1.0115139055 \backslash \mathrm{H}, 1.2820826444,-1.31666716$ $23,1.1582248837 \mathrm{\backslash H},-2.3157006187,-1.0335980271,2.2017927452 \mathrm{H},-0.7450734263,-0.2595296674,2.48$ 72854893।H,-0.9215459528,-2.017339842,2.6358112408\H,-3.331629717,0.4761954307, 1.3307441608। $\mathrm{H},-3.5793447039,1.9220094013,0.3559087207 \mathrm{H},-2.1035812062,1.7574764182,1.3222259494 \mathrm{H},-1.241$ $9174659,1.3575412601,-2.3518145131 \backslash \mathrm{H},-1.3705843869,2.5680757151,-1.0625423025 \backslash \mathrm{H},-2.825671396$ $9,2.0530848863,-1.9296045427 \backslash \mathrm{C}, 1.2900245487,1.0770702141,-0.082793271 \backslash \mathrm{C}, 2.2149197438,1.41399$ $01125,1.0805873394 \backslash \mathrm{H}, 1.8642174332,2.3101016337,1.6000600762 \backslash \mathrm{H}, 3.2284585692,1.6014456004,0.7$ $170763349 \backslash \mathrm{H}, 2.2367429885,0.5922439611,1.8011343779 \backslash \mathrm{H}, 1.5995881638,0.1378825075,-0.549055958$ $3 \backslash \mathrm{C}, 1.3039284269,2.1528331528,-1.1494893872 \backslash \mathrm{C}, 1.0320425265,3.4870093101,-0.8293438914 \backslash \mathrm{C}, 1.03$ 1854996,4.4668213928,-1.8171725693/C,1.3125719686,4.1257790631,-3.1418937773/C, 1.594238493, 2.8019405323,-3.4686158897\C, $1.588700126,1.8230392486,-2.4740577032 \backslash \mathrm{H}, 0.7924705007,3.754825$ 
$4061,0.1966982108 \backslash \mathrm{H}, 0.8104053822,5.4975087361,-1.5572294698 \mathrm{H}, 1.3122654706,4.8901493277,-3.9$ $127294878 \backslash \mathrm{H}, 1.812497435,2.528761541,-4.4964922041 \backslash \mathrm{H}, 1.7921221524,0.7861641233,-2.7308764212$

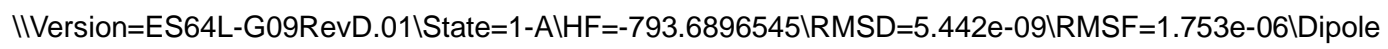
$=-0.0270816,-0.1816393,-0.0989213 \backslash$ Quadrupole $=-1.9354429,2.2848622,-0.3494193,0.1081213,-0.2456$ $817,-0.9034481 \mathrm{PG}=\mathrm{C} 01[\mathrm{X}(\mathrm{C} 17 \mathrm{H} 27 \mathrm{~N} 1 \mathrm{O} 1)] \backslash @$

\section{Alkoxyam radcat}

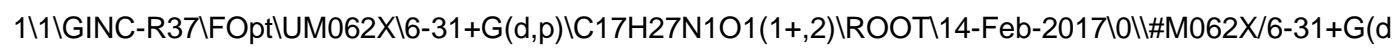
,p) INT(grid=ultrafine) OPT IOP(2/17=4) Freq=noraman maxdisk=536870912॥|TEMPO-STY.RADCAT.3.fre q $\mid 11,2 \backslash \mathrm{C}, 2.8261004456,0.6262850424,-0.6827181292 \backslash \mathrm{N}, 1.3835688491,0.260987018,-0.3900590034 \backslash \mathrm{C}, 0$. $8791224423,-0.9883350525,0.3024695166 \backslash \mathrm{C}, 1.963905989,-2.0850397083,0.2293992481 \backslash \mathrm{C}, 3.39587393$ $35,-1.6031819714,0.3875904765 \backslash \mathrm{C}, 3.6848093563,-0.6383247732,-0.7514776748 \backslash \mathrm{C}, 2.8330911082,1.34$ 60905265,-2.0352724433\C,3.3106910713,1.5673063752,0.4336628823\C,0.5988362248,-0.603122998 $5,1.7648859749 \mid \mathrm{C},-0.3581427043,-1.5740507869,-0.399603253 \backslash 0,0.7116038525,1.3887428517,-0.3101$ $331939 \backslash \mathrm{H}, 1.877360761,-2.598702155,-0.7354243728 \backslash \mathrm{H}, 1.697694274,-2.8166335175,0.9986302844 \backslash \mathrm{H}, 4$. 0723088852,-2.4598681111, $0.3363669417 \mathrm{H}, 3.5608673649,-1.1328446477,1.3632071904 \mathrm{H}, 3.5114587$ $247,-1.1470045829,-1.7080831708 \mathrm{H}, 4.7279507377,-0.3091664359,-0.7525515909 \backslash \mathrm{H}, 2.3685293433,0.7$ $28452423,-2.8096323156 \backslash \mathrm{H}, 3.8727336534,1.5250185797,-2.3212669047 \mathrm{WH}, 2.3201386387,2.307935725$

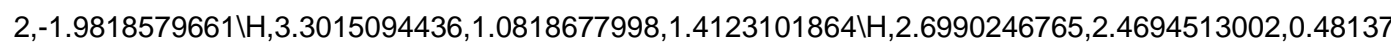
$68743 \backslash \mathrm{H}, 4.3398454804,1.8554822542,0.205673084 \backslash \mathrm{H}, 1.5234872729,-0.3479910073,2.2884525618 \mathrm{H}, 0$. $148163381,-1.4656627498,2.2622082929 \backslash \mathrm{H},-0.0863454305,0.2424822486,1.8542342897 \backslash \mathrm{H},-0.1837648$ $371,-1.672258786,-1.4739654659 \backslash \mathrm{H},-1.2863294948,-1.0319454043,-0.2306077296 \backslash \mathrm{H},-0.4993146723,-2$. $5814148681,0.0013970548 \mathrm{IC},-0.7643902838,1.5360177704,-0.4556574689 \mathrm{IC},-1.0966406811,1.366025$ $8813,-1.9264220146 \mathrm{H},-0.5947536859,2.1310129997,-2.5229276818 \mathrm{H},-2.1753217085,1.4868266083,-2$ $.0542507576 \mid \mathrm{H},-0.8080874674,0.3790308888,-2.2945623759 \mathrm{H},-1.2478369433,0.7887381457,0.169257$ 7625IC,-1.0400310251,2.9024105378,0.1092587643IC,-0.4612865384,4.0432181603,-0.4566936291IC, $-0.73497845,5.2986214009,0.0754339486 \backslash \mathrm{C},-1.5959630593,5.4224331883,1.1673829975 \backslash \mathrm{C},-2.1762452$ $121,4.2893862085,1.7315120078 \backslash \mathrm{C},-1.891679999,3.0294086918,1.2070947154 \backslash \mathrm{H}, 0.2076840285,3.9531$ $944122,-1.3087358039 \backslash \mathrm{H},-0.2828805636,6.1818020648,-0.3632440617 \backslash \mathrm{H},-1.8136774739,6.4035912843$ ,1.5759592796ไH,-2.8464813356,4.3833152078,2.5791684007/H,-2.3443883723,2.145401961, 1.650124 2719||Version=ES64L-G09RevD.01/State $=2-A \backslash H F=-793.4221577 \backslash S 2=0.75452|S 2-1=0| S 2 A=.0.750017 \backslash \mathrm{R}$ $\mathrm{MSD}=4.838 \mathrm{e}-09 \backslash \mathrm{RMSF}=2.656 \mathrm{e}-06 \backslash \mathrm{Dipole}=0.4716368,-1.2725381,-0.1813255 \backslash$ Quadrupole $=1.071663,8.0$ $455327,-9.1171957,-7.4252716,-4.6228971,0.4465725 \backslash P G=C 01$ [X(C17H27N1O1)]1@

\section{Alkoxyam radcat $\mathrm{ClO}_{4}$}

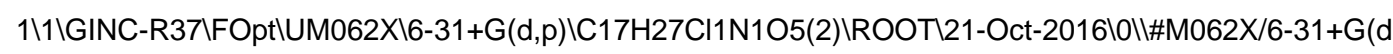
,p) INT(grid=ultrafine) OPT IOP(2/17=4) Freq=noraman maxdisk=536870912॥ITEMPO.CIO4.UN.freq\|I0,2। C,2.2309965353,-1.2818654961,-0.3074281374|C, $1.2402037427,-0.742212905,-1.3296804034 \mid C,-0.164$ 453852,-1.3463945818,-1.2026734698IN,-0.5852146814,-1.3151326076,0.2336862946lC, 0.3303651357 ,-1.5603411233,1.39677151711C, $1.7175600308,-0.9746983625,1.0925544612 \backslash 0,-1.8647457172,-1.6382$ 838905,0.39743291081H,2.4024729297,-2.3572652185,-0.44318080181H,1.5746027043,-0.9496112167, $-2.3520984863 \mid \mathrm{H}, 1.1632777627,0.3443499937,-1.2138760723 \backslash \mathrm{C},-0.2099307398,-2.8143110398,-1.6773$ 595421।C,-1.1537183176,-0.5224290441,-2.0301595583।C,0.3830392923,-3.0940288647,1.5801802552 IC,- $-0.1975193685,-0.9272867674,2.6855452276 \backslash \mathrm{H}, 2.3916107332,-1.3768670764,1.8569533732 \backslash \mathrm{H}, 1.678$ 
$9814387,0.1095326415,1.2359798528) \mathrm{H}, 0.6123909262,-3.4113442856,-1.2810681949 \backslash \mathrm{H},-0.128800143$ $3,-2.8066914144,-2.7674037599 \backslash \mathrm{H},-1.1609792804,-3.2793714709,-1.405620531 \backslash \mathrm{H},-1.2600701002,0.481$ $109034,-1.6153545481 \backslash \mathrm{H},-2.1316800353,-1.0024844232,-2.0938914558 \backslash \mathrm{H},-0.7374805858,-0.445950788$ $5,-3.0386528029 \backslash \mathrm{H}, 0.9254949128,-3.5933834393,0.7768987708 \mathrm{H},-0.6260391791,-3.5096456421,1.645$ $1720423 \backslash \mathrm{H}, 0.904465359,-3.2952062861,2.5194435089 \mathrm{H},-0.3067952193,0.1531667388,2.5836663549 \backslash$ $\mathrm{H}, 0.5587720409,-1.1132851871,3.4531941994 \backslash \mathrm{H},-1.1253825995,-1.3907364334,3.0237008654 \backslash 0,1.401$ $3951783,2.4314398372,-0.1006101155 \backslash \mathrm{Cl}, 0.2290277574,2.4261225778,0.7921957513 \backslash \mathrm{O},-0.6020174564$ ,1.2191119238,0.4540819976\O,0.6576221079,2.2912793156,2.1974517405\O,-0.5932996262,3.624297 $5603,0.6018340197 \backslash \mathrm{C},-2.8175859445,-0.6745722395,1.0669295407 \backslash \mathrm{C},-3.6353223689,0.0023247997,-0$. 001573218\C,-3.6485472433,-1.5334321416,1.9999309407\H,-2.2062861112,0.0595785354,1.58735600 $72 \backslash \mathrm{H},-4.3852345768,-0.8967102903,2.4957933751 \backslash \mathrm{H},-4.187564508,-2.3021200423,1.44081242 \backslash \mathrm{H},-3.03$ $55621722,-2.0219632052,2.7594205536 \backslash \mathrm{C},-4.4806725481,-0.7451435468,-0.8295054572 \backslash \mathrm{C},-5.2360730$ $916,-0.1161195742,-1.8133779154 \backslash \mathrm{C},-5.1610483812,1.269711895,-1.9686599825 \backslash \mathrm{C},-4.3284034722,2.01$ $79676071,-1.1405321446 \backslash \mathrm{C},-3.5612475844,1.3873305035,-0.1606448617 \backslash \mathrm{H},-4.531824309,-1.82596282$ $73,-0.7224035637 \backslash \mathrm{H},-5.882126589,-0.7030076954,-2.4586704089 \backslash \mathrm{H},-5.7497083385,1.7617473418,-2.73$ $6657348 \backslash \mathrm{H},-4.2580950537,3.0939803966,-1.2614682523 \backslash \mathrm{H},-2.8774360545,1.9675109444,0.451550725$ $1 \backslash \mathrm{H}, 3.1977366607,-0.7906825185,-0.4478756738 \| \backslash$ Version=ES64L-G09RevD.01 $\backslash$ State $=2-A \backslash H F=-1554.23$ $67223 \backslash S 2=0.754641 \backslash S 2-1=0 . \mid S 2 A=0.750019 \backslash R M S D=7.174 \mathrm{e}-09 \backslash R M S F=2.011 \mathrm{e}-05 \backslash$ Dipole $=-1.1766073,-4$. 2717352,-0.6003193\Quadrupole=4.235549,-9.7092782,5.4737292,-12.3054848,0.1683352,-6.1255623। $\mathrm{PG}=\mathrm{C} 01[\mathrm{X}(\mathrm{C} 17 \mathrm{H} 27 \mathrm{Cl} 1 \mathrm{~N} 1 \mathrm{O} 5)] \| @$

\section{Ethylbenzene cat $\mathrm{ClO}_{4}$}

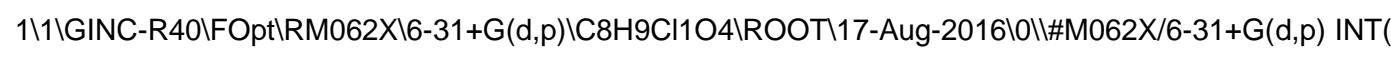
grid=ultrafine) OPT IOP(2/17=4) Freq=noraman maxdisk=4026531840 $\backslash S T Y$ cation.CIO4.freq $\backslash \backslash 0,1 \backslash \mathrm{C},-0.1$ $57802993,0.433100305,0.4995145656 \backslash C, 0.7710893425,-0.4245246149,-0.0871430305 \backslash C, 0.393832879$,$1.7139514707,-0.4661070676 \backslash C,-0.9101810432,-2.1511767929,-0.2562192908 \backslash C,-1.8428599258,-1.295$ $9218234,0.333944751 \backslash \mathrm{C},-1.4684545171,-0.0108742457,0.7076050984 \backslash \mathrm{H}, 1.791898616,-0.0981560804,-$ 0.2562877443\H,1.1235920034,-2.3744073153,-0.9232830005\H,-1.2016254595,-3.1546207723,-0.5496 $4157 \backslash \mathrm{H},-2.8612049192,-1.6317073828,0.5010125232 \backslash \mathrm{H},-2.1894613639,0.6575199013,1.1722412322 \backslash \mathrm{C}$, $0.1935959837,1.8466887702,0.9091041137 \backslash \mathrm{C}, 1.6167419527,2.3012562731,0.6586577977 \backslash \mathrm{H},-0.517790$ $6001,2.5355562054,0.4383036219 \backslash \mathrm{H}, 1.7440366102,3.3222694478,1.021462457 \backslash \mathrm{H}, 2.3248292604,1.650$ $3321301,1.1772769891 \backslash \mathrm{H}, 1.8315168424,2.2819595021,-0.4130125543 \backslash 0,-0.1850876821,4.3691096486$ ,2.3803511993।Cl,-0.9108031561,3.2085405385,2.8587786307\O,-2.2164379789,3.0692105941,2.24268 6472\O,-0.9033297554,3.0395043389,4.2873653079\O,-0.0506340959,1.8962628433,2.3633694985॥Ve

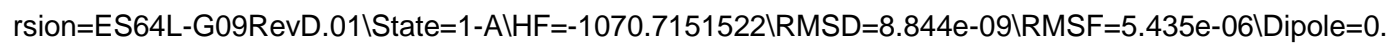
$5732516,-0.8864279,-1.2136852 \backslash$ Quadrupole=3.1839809,0.752558,-3.9365389,1.8963861,-1.8099741,0. 2570125\PG=C01 [X(C8H9Cl1O4)]॥@

\section{Perchlorate}

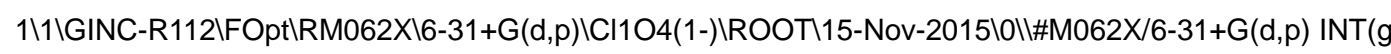
rid=ultrafine) OPT IOP(2/17=4) Freq=noraman maxdisk=134217728 $\backslash \backslash \mathrm{ClO} 4$.anion.freq $\mid l-1,1 \backslash \mathrm{O}, 0.85520440$ 6,0.855204406,-0.855204406\CI,0.,0.,0.1O,0.855204406,-0.855204406,0.855204406।O,-0.855204406,0.8 $55204406,0.85520440610,-0.855204406,-0.855204406,-0.855204406|| V e r s i o n=E S 64 L-G 09 R e v D .01 \backslash S t a t$

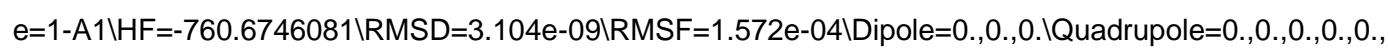




\section{ACN}

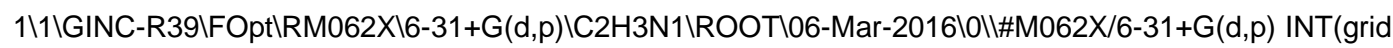

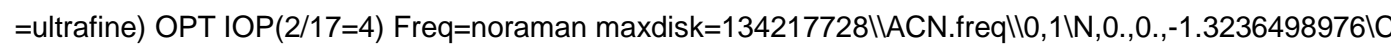
$, 0 ., 0 .,-0.168223276 \backslash \mathrm{C}, 0 ., 0 ., 1.294497059 \backslash \mathrm{H}, 0.5132913608,0.8890467161,1.6643709193 \backslash \mathrm{H}, 0.5132913608$ ,-0.8890467161,1.6643709193\H,-1.0265827217,0., 1.6643709193||Version=ES64L-G09RevD.01।State=1 $-\mathrm{A} 1 \mathrm{HF}=-132.7007779|\mathrm{RMSD}=4.068 \mathrm{e}-09| \mathrm{RMSF}=7.617 \mathrm{e}-06|\mathrm{Dipole}=0 ., 0 ., 1.5798498|$ Quadrupole $=0.76818$ $38,0.7681838,-1.5363677,0 ., 0 ., 0 . \mid P G=C 03 V[C 3(C 1 C 1 N 1), 3 S G V(H 1)] \| @$

\section{Ethylbenzene cat ACN}

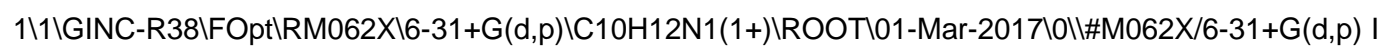
NT(grid=ultrafine) OPT IOP(2/17=4) Freq=noraman maxdisk=268435456\|ISTY_cation.ACN.freq |11,11C,-2. 6021079791,0.4019254179,-1.0776650983\C,-1.0821228555,0.5583466528,-1.0636829645\C,-0.310999 $5566,-0.6255180277,-0.516273439 \backslash \mathrm{C},-0.6039275312,-1.1414298975,0.7494975553 \backslash \mathrm{C}, 0.100284591,-2.2$ 418085915,1.2276906541।C, $1.0904021714,-2.8330331069,0.4411857009 \mid \mathrm{C}, 1.3799156651,-2.32068456$ $7,-0.8208233871 \backslash \mathrm{C}, 0.6830751397,-1.2117259652,-1.299065585 \mathrm{WN},-0.7427106299,1.7407484662,-0.259$ 1572668\C,-0.4611223694,2.6342091898,0.3996626005\C,-0.0954693396,3.7653612409,1.2349788913\ $\mathrm{H},-2.8374107047,-0.4572642253,-1.7083667533 \mathrm{H},-3.0883917181,1.2878799641,-1.4907484004 \mathrm{lH},-2.9$ $872833052,0.207003334,-0.0751074208 \mid \mathrm{H},-0.7161768256,0.809084377,-2.064026426 \backslash \mathrm{H},-1.3812577781$ ,-0.6939889254,1.3645815236\H,-0.1276736678,-2.6435055085,2.2091735697\\,1.6332091686,-3.6952 $943986,0.8135635873|\mathrm{H}, 2.1464057758,-2.7810504105,-1.4347875556| \mathrm{H}, 0.9100316835,-0.8131405374$, $-2.2844048861 \backslash \mathrm{H},-0.9987272369,4.1866854851,1.6822423752 \backslash \mathrm{H}, 0.4000974939,4.5177300797,0.61680$

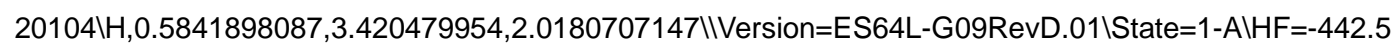
935175\RMSD $=6.748 \mathrm{e}-09 \backslash \mathrm{RMSF}=2.008 \mathrm{e}-06 \backslash \mathrm{Dipole}=-0.3265278,3.1551209,0.4871912 \backslash$ Quadrupole $=-9.8$ 611227,16.3732483,-6.5121256,-4.0292303,0.2808494,5.5364761 $\mathrm{PG}=\mathrm{C} 01$ [X(C10H12N1)]/@

\section{DCM}

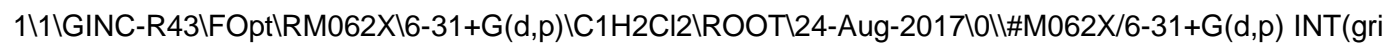
$d=u$ ltrafine) $\mathrm{OPT} \operatorname{IOP}(2 / 17=4)$ Freq=noraman maxdisk=8GB\|ISTY_cation.ACN.freq || $0,1 \backslash \mathrm{Cl}, 0 ., 0.14676570$

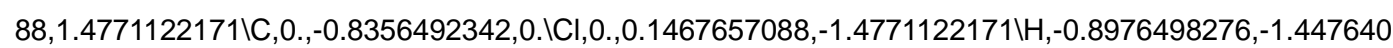

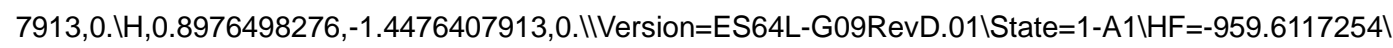
$\mathrm{RMSD}=1.105 \mathrm{e}-09|\mathrm{RMSF}=1.928 \mathrm{e}-04| \mathrm{Dipole}=0 .,-0.7515486,0 . \mid$ Quadrupole $=0.3059995,1.5530245,-1.8590$ 241,0.,0.,0.|PG=C02V [C2(C1),SGV(Cl2),SGV'(H2)]॥@

\section{Ethylbenzene cat ACN}

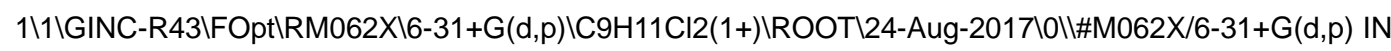
$\mathrm{T}$ (grid=ultrafine) OPT IOP(2/17=4) Freq=noraman maxdisk=8GB $\mid S T Y$ cation.ACN.freq $\| 1,1 \backslash \mathrm{C}, 0.2316860$ $234,-1.2107405518,1.897808434 \mid \mathrm{C}, 0.5653121873,0.1188764101,2.1227613126 \backslash \mathrm{C}, 0.3339158712,0.9269$ 924348,3.3246558297\C,-0.4080687362,-2.0227023582,2.8828739466\C,-0.7620266567,-3.3172220347 ,2.5753315688/C,-0.4934434389,-3.8243663491,1.2916783544IC,0.1355293633,-3.0507804615,0.3062 556284IC, $0.4975911934,-1.7544133819,0.6034587534|\mathrm{Cl},-1.6339254677,1.4517007251,0.0645601008|$ $\mathrm{H}, 1.0541952186,0.6372558802,1.2967478366) \mathrm{H},-0.1239522655,0.3988334855,4.1573765802 \backslash \mathrm{H},-0.3010$ $492317,1.776487426,3.0324705674 \backslash \mathrm{H}, 1.2844291839,1.378468339,3.6380916626 \backslash \mathrm{H},-0.6180585985,-1.6$ 
$228207707,3.8685211001 \backslash \mathrm{H},-1.2483751661,-3.9448473233,3.3132816261 \backslash \mathrm{H},-0.7821374187,-4.8452872$

$114,1.0581876065 \mathrm{H}, 0.3281259131,-3.4689173708,-0.6748842914 \backslash \mathrm{H}, 0.97521318,-1.1267417752,-0.144$ 0420311\C,-3.1592575157,0.5299634081,-0.008716558ICI,-3.0672412129,-1.0022630148,0.883621702 $2 \backslash \mathrm{H},-3.3636818017,0.3100202954,-1.0526242756 \backslash \mathrm{H},-3.9390532239,1.1404306993,0.4374692456|| V e r s i$ on=ES64L-G09RevD.01 \State=1-A $\backslash H F=-1269.4738935 \backslash R M S D=2.793 e-09 \backslash R M S F=6.266 e-06 \backslash$ Dipole=1.23 29293,-0.400653,0.7606595\Quadrupole=-3.3359816,4.8670465,-1.5310649,-0.6133265,4.2470657,2.21 29502\PG=C01 [X(C9H11Cl2)]॥@

\section{S9. References}

(1) Yapici, N. B.; Jockusch, S.; Moscatelli, A.; Mandalapu, S. R.; Itagaki, Y.; Bates, D. K.; Wiseman, S.; Gibson, K. M.; M.; Turro, N. J.; Bi, L. Org. Lett. 2011, 14, 50-53.

(2) Danko, M.; Szabo, E.; Hrdlovic, P. Dyes Pigments 2011, 90, 129-138.

(3) (a) Ciampi, S.; Böcking, T.; Kilian, K. A.; James, M.; Harper, J. B.; Gooding, J. J. Langmuir 2007, 23, 9320-9329;

(b) Ciampi, S.; Eggers, P. K.; Le Saux, G.; James, M.; Harper, J. B.; Gooding, J. J. Langmuir 2009, 25, 2530-2539.

(4) Meldal, M.; Tornøe, C. W. Chem. Rev. 2008, 108, 2952-3015.

(5) Chen, L.; Xu, Z.; Li, J.; Zhou, B.; Shan, M.; Li, Y.; Liu, L.; Li, B.; Niu, J. RSC Adv. 2014, 4, 1025-1031.

(6) Ciampi, S.; Guan, B.; Darwish, N. A.; Zhu, Y.; Reece, P. J.; Gooding, J. J. Phys. Chem. Chem. Phys. 2012, 14, 16433-16439.

(7) (a) Ciampi, S.; James, M.; Michaels, P.; Gooding, J. J. Langmuir 2011, 27, 6940-6949; (b) Devadoss, A.; Chidsey, Chidsey, C. E. J. Am. Chem. Soc. 2007, 129, 5370-5371.

(8) (a) Liu, H.; Duclairoir, F.; Fleury, B.; Dubois, L.; Chenavier, Y.; Marchon, J.-C. Dalton T. 2009, 3793-3799; (b) Qin, Qin, G.; Santos, C.; Zhang, W.; Li, Y.; Kumar, A.; Erasquin, U. J.; Liu, K.; Muradov, P.; Trautner, B. W.; Cai, C. J. Am. Chem. Soc. 2010, 132, 16432-16441.

(9) Caro, J.; Fraxedas, J.; Jürgens, O.; Santiso, J.; Rovira, C.; Veciana, J.; Figueras, A. Adv. Mater. 1998, 10, 608-610.

(10) (a) Grobman, W.-D.; Pollak, R. A.; Eastman, D.; Maas Jr, E.; Scott, B. Phys. Rev. Lett. 1974, 32, 534-537; (b) Fraxedas, J.; Caro, J.; Figueras, A.; Gorostiza, P.; Sanz, F. J. Vac. Sci. Technol. A 1998, 16, 2517-2523.

(11) Wagner, H.; Brinks, M. K.; Hirtz, M.; Schäfer, A.; Chi, L.; Studer, A. Chem. Eur. J. 2011, 17, 9107-9112.

(12) Wang, Y.; Rogers, E. I.; Compton, R. G. J. Electroanal. Chem. 2010, 648, 15-19.

(13) Witting, P. K.; Douglas, D.; Mauk, A. G. J. Biol. Chem. 2000, 275, 20391-20398.

(14) Aragonès, A. C.; Haworth, N. L.; Darwish, N.; Ciampi, S.; Bloomfield, N. J.; Wallace, G. G.; Diez-Perez, I.; Coote, M. L. Nature 2016, 531, 88-91.

(15) Frisch, J.; Trucks, G. W.; Schegel, H. B.; Scuseria, G. E.; Robb, M. A.; Cheeseman, J. R.; Scalmani, G.; Barone, V.; Mennucci, B.; Petersson, G. A.; Nakatsuji, H.; Caricato, M.; Li, X.; Hratchian, H. P.; Izmaylov, A.; Bloino, J.; Zheng, G.; Sonnenberg, J. L.; Hada, M.; Ehara, M.; Toyota, K.; Fukuda, R.; Hasegawa, J.; Ishida, M.; Nakajima, N.; Honda, Y.; Kitao, O.; Nakai, H.; Vreven, T.; Montgomery, J. A.; Jr. Peralta, J. E.; Ogliaro, F.; Bearpark, M.; Heyd, J. J.; Brothers, E.; E.; Kudin, K. N.; Staroverov, V. N.; Keith, T.; Kobayashi, R.; Normand, J.; Raghavachari, K.; Rendell, A.; Burant, J. C.; lyengar, S. S.; Tomasi, J.; Cossi, M.; Rega, N.; Millam, J. M.; Klene, M.; Knox, J. E.; Cross, J. B.; Bakken, V.; Adamo, C.; C.; Jaramillo, J.; Gomperts, R.; Stratmann, R. E.; Yazyev, O.; Austin, A. J.; Cammi, R.; Pomelli, C.; Ochterski, J. W.; Martin, R. L.; Morokuma, K.; Zakrzewski, V. G.; Voth, G. A.; Salvador, P.; Dannenberg, J. J.; Dapprich, S.; Daniels, A. D.; Farkas, O.; Foresman, J. B.; Ortiz, J. V.; Cioslowski, J.; Fox, J. Rev. D. Gaussian 09, Gaussian, Inc., Wallingford CT. CT. 2013. 
(16) (a) Shaik, S.; Mandal, D.; Ramanan, R. Nat. Chem. 2016, 8, 1091-1098; (b) Lai, W.; Chen, H.; Cho, K. B.; Shaik, S. J. Phys, Chem. Lett. 2010, 1, 2082-2087; (c) Meir, R.; Chen, H.; Lai, W.; Shaik, S. ChemPhysChem 2010, 11, 301-310.

(17) (a) Gryn'ova, G.; Coote, M. L. J. Am. Chem. Soc. 2013, 135, 15392-15403; (b) Gryn'ova, G.; Marshall, D. L.; Blanksby, S. J.; Coote, M. L. Nat. Chem. 2013, 5, 474-481.

(18) Gryn'Ova, G.; Smith, L. M.; Coote, M. L. Phys. Chem. Chem. Phys. 2017, 19, 22678-22683.

(19) Zhao, Y.; Truhlar, D. G. Theor. Chem. Acc. 2008, 120, 215-241.

(20) (a) Hehre, W. J.; Ditchfield, R.; Pople, J. A. J. Chem. Phys. 1972, 56, 2257-2261; (b) Dill, J. D.; Pople, J. A. J. Chem. Phys. 1975, 62, 2921-2923; (c) Clark, T.; Chandrasekhar, J.; Spitznagel, G. W.; Schleyer, P. V. R. J. Comput. Chem. 1983, 4, 294-301; (d) Francl, M. M.; Pietro, W. J.; Hehre, W. J.; Binkley, J. S.; Gordon, M. S.; DeFrees, D. J.; Pople, J. A. J. Chem. Phys. 1982, 77, 3654-3665; (e) Frisch, M. J.; Pople, J. A.; Binkley, J. S. J. Chem. Phys. 1984, 80, 3265-3269.

(21) Alecu, I.; Zheng, J.; Zhao, Y.; Truhlar, D. G. J. Chem. Theory Comput. 2010, 6, 2872-2887.

(22) Curtiss, L. A.; Raghavachari, K.; Redfern, P. C.; Baboul, A. G.; Pople, J. A. Chem. Phys. Lett. 1999, 314, 101-107.

(23) (a) Coote, M. L.; Krenske, E. H.; Izgorodina, E. I. Macromol. Rapid Commun. 2006, 27, 473-497; (b) Izgorodina, E. I.; Brittain, D. R.; Hodgson, J. L.; Krenske, E. H.; Lin, C. Y.; Namazian, M.; Coote, M. L. J. Phys. Chem. A 2007, 111, 10754-10768.

(24) Marenich, A. V.; Cramer, C. J.; Truhlar, D. G. J. Phys. Chem. B 2009, 113, 6378-6396.

(25) (a) Klamt, A. J. Phys, Chem. 1995, 99, 2224-2235; (b) Klamt, A.; Jonas, V.; Bürger, T.; Lohrenz, J. C. J. Phys. Chem. A 1998, 102, 5074-5085; (c) Klamt, A., COSMO-RS: from quantum chemistry to fluid phase thermodynamics and drug design. Elsevier: 2005.

(26) Louwen, J. N.; Pye, C. C.; Van Lenthe, E.; McGarrity, E. S.; Xiong, R.; Sandler, S. I.; Burnett, R. I., In http://www.scm.com, ADF2014 COSMO-RS, SCM, Theoreoretical Chemistry, Vrije Universiteit, Amsterdam, The Netherlands: 2014.

(27) Pye, C. C.; Ziegler, T.; Van Lenthe, E.; Louwen, J. N. Can. J. Chem. 2009, 87, 790-797.

(28) (a) Gryn'ova, G.; Barakat, J. M.; Blinco, J. P.; Bottle, S. E.; Coote, M. L. Chem-Eur. J. 2012, 18, 7582-7593; (b) Zhang, L.; Vogel, Y. B.; Noble, B. B.; Gonçales, V. R.; Darwish, N.; Brun, A. L.; Gooding, J. J.; Wallace, G. G.; Coote, M. M. L.; Ciampi, S. J. Am. Chem. Soc. 2016, 138, 9611-9619.

(29) (a) Werner, H. J.; Knowles, P. J.; Knizia, G.; Manby, F. R.; Schütz, M. WIREs Comput. Mol. Sci. 2012, 2, 242-253; 242-253; (b) Werner, H. J.; Knowles, P. J.; Knizia, G.; Manby, F. R.; Schütz, M.; Celani, P.; Györffy, W.; Kats, D.; Korona, T.; Lindh, R.; Mitrushenkov, A.; Rauhut, G.; Shamasundar, K. R.; Adler, T. B.; Amos, R. D.; Bernhardsson, A.; Berning, A.; Cooper, D. L.; Deegan, M. J. O.; Dobbyn, A. J.; Eckert, F.; Goll, E.; Hampel, C.; Hesselmann, A.; Hetzer, G.; G.; Hrenar, T.; Jansen, G.; Köppl, C.; Liu, Y.; Lloyd, A. W.; Mata, R. A.; May, A. J.; McNicholas, S. J.; Meyer, W.; Mura, M. E.; Nicklass, A.; O'Neill, D. P.; Palmieri, P.; Peng, D.; Pflüger, K.; Pitzer, R.; Reiher, M.; Shiozaki, T.; Stoll, H.; Stone, Stone, A. J.; Tarroni, R.; Thorsteinsson, T.; Wang, M. MOLPRO, version 2015.1, a package of ab initio programs, http://www.molpro.net 2015.

(30) Shao, Y.; Gan, Z.; Epifanovsky, E.; Gilbert, A. T. B.; Wormit, M.; Kussmann, J.; Lange, A. W.; Behn, A.; Deng, J.; Feng, X. Mol. Phys. 2015, 113, 184-215.

(31) Namazian, M.; Lin, C. Y.; Coote, M. L. J. Chem. Theory Comput. 2010, 6, 2721-2725.

(32) Bartmess, J. E. J. Phys. Chem. 1994, 98, 6420-6424.

(33) Namazian, M.; Coote, M. L. J. Phys. Chem. A 2007, 111, 7227-7232.

(34) Pavlishchuk, V. V.; Addison, A. W. Inorg. Chim. Acta 2000, 298, 97-102.

(35) Su, B.; Girault, H. H. J. Phys. Chem. B 2005, 109, 11427-11431. 